\title{
Carbocyclization of Heterosubstituted Alkynes via the Memory of Chirality: Access to Ca-substituted Proline Derivatives
}

\author{
Shenpeng Tan, Feng Li, Soojun Park, and Sanghee Kim* \\ College of Pharmacy, Seoul National University, 1 Gwanak-ro, Gwanak-gu, Seoul 08826, \\ Republic of Korea \\ E-mail: pennkim@snu.ac.kr
}

Table of Contents

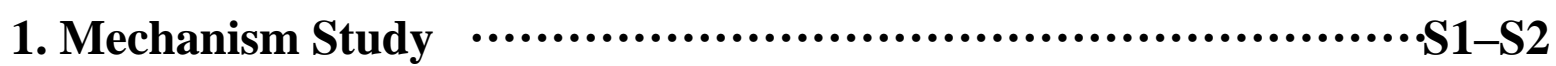

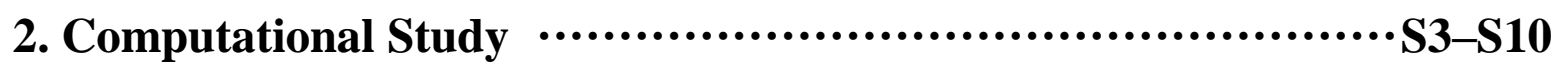

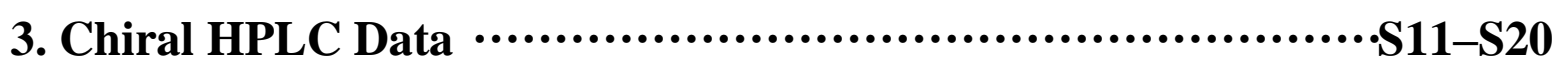

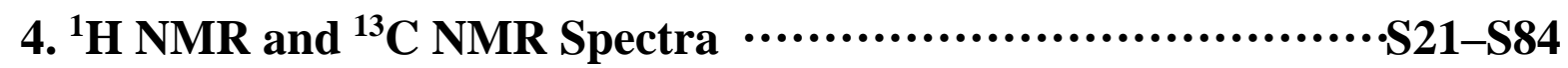




\section{Mechanism Study}

\subsection{Incomplete MOC reaction}
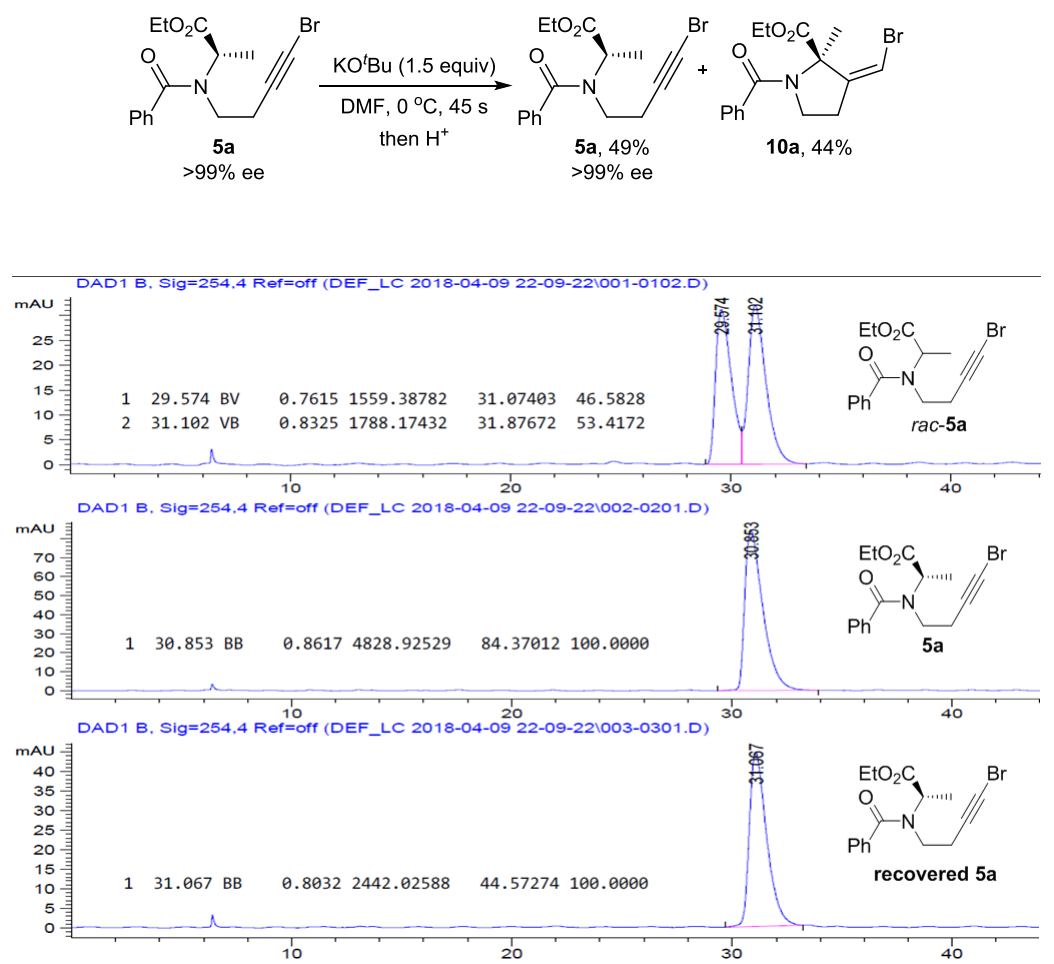

Figure S1. Chiral HPLC chromatograms of MOC substrate.

\subsection{Incomplete $\mathrm{MOC}$ reaction quenched with $\mathrm{D}_{2} \mathrm{O} / \mathrm{AcOD}$}
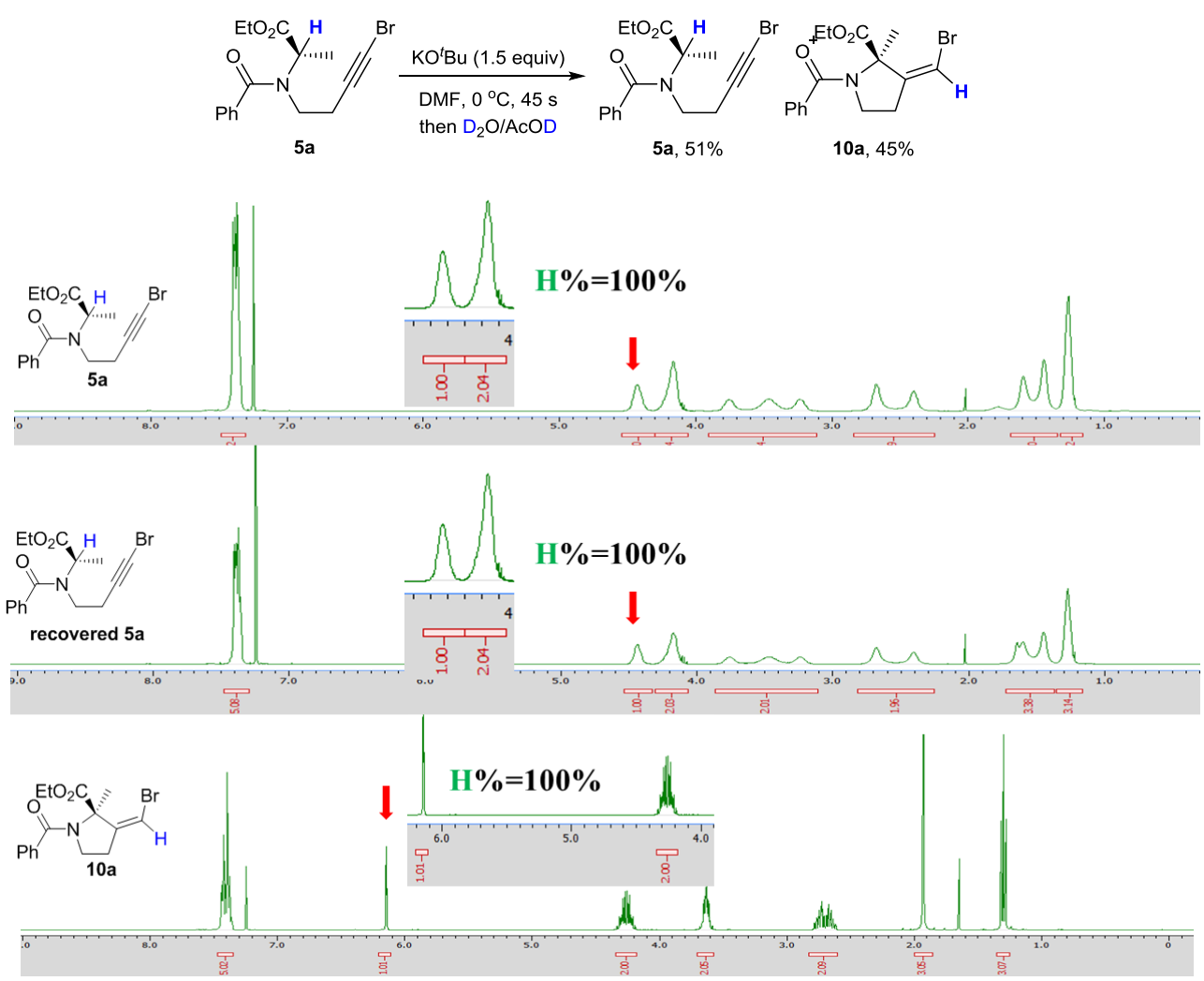

Figure S2. ${ }^{1} \mathrm{H}$ NMR spectra of $\mathbf{5 a}$, recovered 5a, and 10a after the incomplete MOC reaction. 


\subsection{Incomplete MOC reaction with $r a c$-[D]-5a}
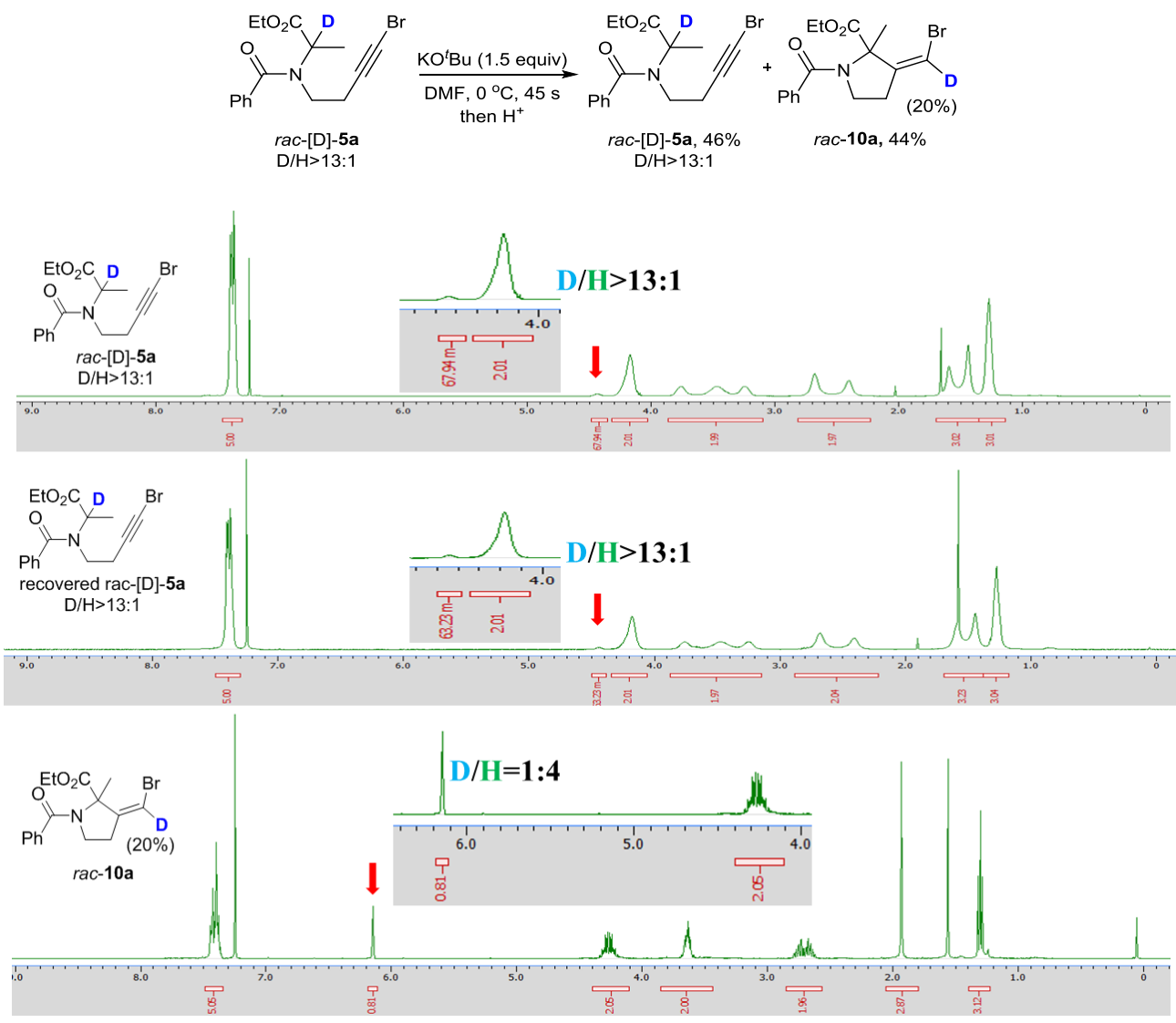

Figure S3. ${ }^{1} \mathrm{H}$ NMR spectra of $r a c-[\mathrm{D}]-5 \mathbf{a}$, recovered $r a c$-[D]-5a, and $r a c-10 a$ after the incomplete MOC reaction. 


\section{Computational Study}

\subsection{Procedure for internal methyl alkyne calculation}

DFT calculations were carried out at the wb97xd level of theory with the $6-31+G(d)$ basis set in Gaussian 09 . Optimization and frequency calculations were performed to determine the Gibbs free energy (reported at $298.15 \mathrm{~K}$ and $1 \mathrm{~atm})$. Molecules were modeled in the solvent phase (DMF, polarizable continuum model (PCM)).

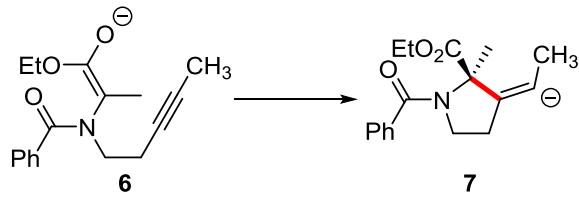

Table S1. Cartesian coordinates of the Z-enolate 6

\begin{tabular}{cccccccc}
\hline Atom & $\mathrm{X}$ & $\mathrm{Y}$ & $\mathrm{Z}$ & $\mathrm{Atom}$ & $\mathrm{X}$ & $\mathrm{Y}$ & $\mathrm{Z}$ \\
\hline $\mathrm{C}$ & -0.37579 & 0.943061 & -0.12737 & $\mathrm{C}$ & -2.32093 & -1.45271 & 0.994255 \\
$\mathrm{C}$ & -0.98126 & 1.980386 & 0.831571 & $\mathrm{C}$ & -1.29496 & -2.63326 & -0.8447 \\
$\mathrm{C}$ & 1.73297 & 0.49166 & 1.096971 & $\mathrm{C}$ & -3.5665 & -1.956 & 0.622362 \\
$\mathrm{H}$ & -1.17451 & 0.411081 & -0.64771 & $\mathrm{H}$ & -2.23599 & -0.80264 & 1.861106 \\
$\mathrm{H}$ & 0.22941 & 1.453324 & -0.88196 & $\mathrm{C}$ & -2.54171 & -3.12759 & -1.22261 \\
$\mathrm{H}$ & -0.16853 & 2.516544 & 1.335133 & $\mathrm{H}$ & -0.40628 & -2.90082 & -1.41035 \\
$\mathrm{H}$ & -1.55939 & 1.470273 & 1.611218 & $\mathrm{C}$ & -3.67995 & -2.78993 & -0.48973 \\
$\mathrm{~N}$ & 0.491253 & -0.00671 & 0.563976 & $\mathrm{H}$ & -4.44821 & -1.69566 & 1.201084 \\
$\mathrm{C}$ & 2.794271 & 0.747135 & 0.260381 & $\mathrm{H}$ & -2.62411 & -3.77908 & -2.08797 \\
$\mathrm{O}$ & 3.959884 & 1.132875 & 0.570635 & $\mathrm{H}$ & -4.651 & -3.17899 & -0.78226 \\
$\mathrm{O}$ & 2.492316 & 0.596893 & -1.1084 & $\mathrm{C}$ & 1.815028 & 0.61639 & 2.590414 \\
$\mathrm{C}$ & 3.540847 & 0.092318 & -1.92173 & $\mathrm{H}$ & 2.785103 & 1.034853 & 2.875735 \\
$\mathrm{H}$ & 3.245169 & 0.319838 & -2.95062 & $\mathrm{H}$ & 1.033838 & 1.273325 & 3.004282 \\
$\mathrm{H}$ & 4.472485 & 0.624518 & -1.70302 & $\mathrm{H}$ & 1.710232 & -0.35702 & 3.094921 \\
$\mathrm{C}$ & 3.72231 & -1.40869 & -1.73914 & $\mathrm{C}$ & -1.83838 & 2.93089 & 0.116676 \\
$\mathrm{H}$ & 4.506323 & -1.78275 & -2.40791 & $\mathrm{C}$ & -2.54034 & 3.694127 & -0.50766 \\
$\mathrm{H}$ & 4.009946 & -1.64029 & -0.70791 & $\mathrm{C}$ & -3.38986 & 4.618864 & -1.26221 \\
$\mathrm{H}$ & 2.790955 & -1.94026 & -1.96381 & $\mathrm{H}$ & -4.44881 & 4.442593 & -1.04783 \\
$\mathrm{C}$ & 0.187603 & -1.3184 & 0.688823 & $\mathrm{H}$ & -3.1616 & 5.659132 & -1.00884 \\
$\mathrm{O}$ & 0.972294 & -2.15875 & 1.154497 & $\mathrm{H}$ & -3.2386 & 4.493101 & -2.33913 \\
$\mathrm{C}$ & -1.18021 & -1.78529 & 0.259375 & & & & \\
\hline & & & & & & & \\
\hline
\end{tabular}


Table S2. Cartesian coordinates of the intermediate 7

\begin{tabular}{cccccccc}
\hline Atom & $\mathrm{X}$ & $\mathrm{Y}$ & $\mathrm{Z}$ & $\mathrm{Atom}$ & $\mathrm{X}$ & $\mathrm{Y}$ & $\mathrm{Z}$ \\
\hline $\mathrm{C}$ & -0.94295 & 1.893639 & -0.20281 & $\mathrm{C}$ & -2.58597 & -0.4585 & 0.125358 \\
$\mathrm{C}$ & 0.154908 & 2.881667 & 0.158762 & $\mathrm{C}$ & -3.02081 & -0.31281 & -1.19393 \\
$\mathrm{C}$ & 1.058719 & 0.645405 & 0.487398 & $\mathrm{C}$ & -3.52028 & -0.62486 & 1.149554 \\
$\mathrm{H}$ & -1.90627 & 2.1034 & 0.267851 & $\mathrm{C}$ & -4.38316 & -0.33026 & -1.48588 \\
$\mathrm{H}$ & -1.08506 & 1.848631 & -1.2894 & $\mathrm{H}$ & -2.29487 & -0.1933 & -1.99393 \\
$\mathrm{H}$ & 0.005143 & 3.223483 & 1.194565 & $\mathrm{C}$ & -4.88297 & -0.62758 & 0.857697 \\
$\mathrm{H}$ & 0.152031 & 3.761689 & -0.48874 & $\mathrm{H}$ & -3.18011 & -0.74875 & 2.174209 \\
$\mathrm{~N}$ & -0.40602 & 0.614949 & 0.288871 & $\mathrm{C}$ & -5.31619 & -0.48166 & -0.46032 \\
$\mathrm{C}$ & 1.651093 & -0.42514 & -0.43427 & $\mathrm{H}$ & -4.7157 & -0.22291 & -2.51433 \\
$\mathrm{O}$ & 1.318183 & -0.57276 & -1.59565 & $\mathrm{H}$ & -5.6069 & -0.74717 & 1.658597 \\
$\mathrm{O}$ & 2.590306 & -1.16458 & 0.150506 & $\mathrm{H}$ & -6.37843 & -0.48846 & -0.68712 \\
$\mathrm{C}$ & 3.280009 & -2.11713 & -0.67585 & $\mathrm{C}$ & 1.413875 & 0.451261 & 1.968162 \\
$\mathrm{H}$ & 2.55565 & -2.84744 & -1.04831 & $\mathrm{H}$ & 2.492651 & 0.548798 & 2.109771 \\
$\mathrm{H}$ & 3.709666 & -1.5887 & -1.53265 & $\mathrm{H}$ & 0.920362 & 1.23753 & 2.54809 \\
$\mathrm{C}$ & 4.350035 & -2.76585 & 0.174863 & $\mathrm{H}$ & 1.092914 & -0.52285 & 2.345297 \\
$\mathrm{H}$ & 4.897075 & -3.50025 & -0.42478 & $\mathrm{C}$ & 2.622427 & 2.593472 & -0.38804 \\
$\mathrm{H}$ & 5.062576 & -2.01941 & 0.539644 & $\mathrm{C}$ & 3.839809 & 1.677073 & -0.45446 \\
$\mathrm{H}$ & 3.908984 & -3.28071 & 1.033912 & $\mathrm{H}$ & 3.958429 & 0.998081 & 0.406226 \\
$\mathrm{C}$ & 1.445858 & 2.086654 & 0.020504 & $\mathrm{H}$ & 3.80566 & 1.035902 & -1.35227 \\
$\mathrm{C}$ & -1.11675 & -0.51648 & 0.443834 & $\mathrm{H}$ & 4.775161 & 2.245208 & -0.53521 \\
$\mathrm{O}$ & -0.61009 & -1.58277 & 0.823026 & & & & \\
\hline & & & & & & & \\
\hline
\end{tabular}

Table S3. Electronic energies (E), zero-point energies (ZPE), enthalpies $(H)$, and Gibbs free energies (G) (in Hartree, Ha) of the compound calculated at the wb97xd, 6-31+G(d) level of theory. Relative Gibbs free energy is shown in kcal $\mathrm{mol}^{-1}$.

\begin{tabular}{cccccc}
\hline Compound & E & ZPE & H & G & $\Delta G^{a, b}$ \\
\hline $\mathbf{6}(\boldsymbol{Z})$ & -940.079151 & -939.739696 & -939.716574 & -939.794935 & 0.00 \\
$\mathbf{7}$ & -940.080957 & -939.738903 & -939.717472 & -939.788865 & 3.81 \\
\hline
\end{tabular}

${ }^{a} 1 \mathrm{Ha}=627.509391 \mathrm{kcal} \mathrm{mol}^{-1} .{ }^{b}$ Relative energy Gibbs free energy between Z-enolate 6 and intermediate 7. 


\subsection{Procedure for the calculation of $Z$-enolate 8 and intermediate 9}

Density functional theory (DFT) calculations were carried out at the wb97xd level of theory with the 6-31+G(d) basis set in Gaussian 09. Optimization and frequency were performed to calculate the Gibbs free energy (reported at $298.15 \mathrm{~K}$ and $1 \mathrm{~atm})$. molecules were modeled in the solvent phase (DMF, polarizable continuum model (PCM)).

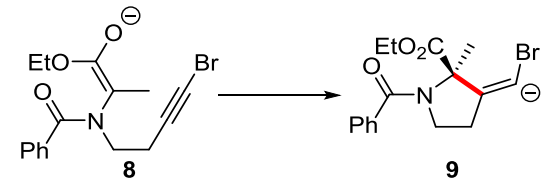

Table S4. Cartesian coordinates of the Z-enolate 8

\begin{tabular}{rcrcrcrc}
\hline Atom & $\mathrm{X}$ & $\mathrm{Y}$ & $\mathrm{Z}$ & Atom & $\mathrm{X}$ & $\mathrm{Y}$ & $\mathrm{Z}$ \\
\hline $\mathrm{C}$ & -0.04654 & -0.27192 & 0.078674 & $\mathrm{O}$ & -3.0478 & 1.594406 & 0.829266 \\
$\mathrm{C}$ & 0.931264 & -0.62912 & 1.210681 & $\mathrm{C}$ & -0.96396 & 2.432254 & 0.091409 \\
$\mathrm{C}$ & -2.14043 & -1.00372 & 1.177588 & $\mathrm{C}$ & 0.04116 & 2.90045 & 0.941714 \\
$\mathrm{H}$ & 0.352555 & 0.5595 & -0.50467 & $\mathrm{C}$ & -1.16649 & 3.053118 & -1.14315 \\
$\mathrm{H}$ & -0.15525 & -1.12664 & -0.59482 & $\mathrm{C}$ & 0.844623 & 3.971941 & 0.555942 \\
$\mathrm{H}$ & 0.565738 & -1.51781 & 1.736986 & $\mathrm{H}$ & 0.193894 & 2.430169 & 1.909587 \\
$\mathrm{H}$ & 0.967245 & 0.18657 & 1.941617 & $\mathrm{C}$ & -0.35703 & 4.118926 & -1.53227 \\
$\mathrm{~N}$ & -1.37101 & 0.061917 & 0.590817 & $\mathrm{H}$ & -1.95224 & 2.695445 & -1.80331 \\
$\mathrm{C}$ & -2.74368 & -1.94745 & 0.374588 & $\mathrm{C}$ & 0.650917 & 4.579309 & -0.68452 \\
$\mathrm{O}$ & -3.43861 & -2.94211 & 0.732043 & $\mathrm{H}$ & 1.621817 & 4.330626 & 1.224758 \\
$\mathrm{O}$ & -2.49829 & -1.75969 & -0.99787 & $\mathrm{H}$ & -0.51312 & 4.590241 & -2.49842 \\
$\mathrm{C}$ & -3.31153 & -2.48982 & -1.90345 & $\mathrm{H}$ & 1.281236 & 5.410069 & -0.98856 \\
$\mathrm{H}$ & -2.7433 & -2.51853 & -2.83879 & $\mathrm{C}$ & -2.28447 & -0.97838 & 2.67127 \\
$\mathrm{H}$ & -3.44532 & -3.51451 & -1.545 & $\mathrm{H}$ & -2.87337 & -1.83932 & 3.002701 \\
$\mathrm{C}$ & -4.66119 & -1.82105 & -2.12371 & $\mathrm{H}$ & -1.31563 & -1.01882 & 3.194683 \\
$\mathrm{H}$ & -5.25445 & -2.39098 & -2.84865 & $\mathrm{H}$ & -2.79614 & -0.06946 & 3.025805 \\
$\mathrm{H}$ & -5.22057 & -1.77119 & -1.18409 & $\mathrm{C}$ & 2.276738 & -0.87677 & 0.696307 \\
$\mathrm{H}$ & -4.53225 & -0.80321 & -2.50814 & $\mathrm{C}$ & 3.382585 & -1.06413 & 0.249197 \\
$\mathrm{C}$ & -1.87871 & 1.314525 & 0.52546 & $\mathrm{Br}$ & 5.024407 & -1.34102 & -0.4137 \\
\hline & & & & & & &
\end{tabular}

Table S5. Cartesian coordinates of the intermediate 9

\begin{tabular}{cccccccc}
\hline Atom & $\mathrm{X}$ & $\mathrm{Y}$ & $\mathrm{Z}$ & Atom & $\mathrm{X}$ & $\mathrm{Y}$ & $\mathrm{Z}$ \\
\hline $\mathrm{C}$ & 0.977296 & -1.17837 & -1.30632 & $\mathrm{C}$ & 1.677444 & -0.10849 & 0.834349 \\
$\mathrm{C}$ & -0.24996 & -2.02622 & -1.59156 & $\mathrm{O}$ & 1.39701 & 0.471026 & 1.890064 \\
$\mathrm{C}$ & -0.68371 & -0.75827 & 0.456084 & $\mathrm{C}$ & 3.09748 & -0.1102 & 0.339958 \\
$\mathrm{H}$ & 1.91256 & -1.72973 & -1.3997 & $\mathrm{C}$ & 3.849151 & -1.28667 & 0.29503
\end{tabular}




\begin{tabular}{lrllllll}
$\mathrm{H}$ & 1.017382 & -0.29984 & -1.96276 & $\mathrm{C}$ & 3.686278 & 1.104953 & -0.01516 \\
$\mathrm{H}$ & -0.08493 & -3.04238 & -1.20624 & $\mathrm{C}$ & 5.183168 & -1.24674 & -0.10491 \\
$\mathrm{H}$ & -0.47255 & -2.09947 & -2.65781 & $\mathrm{H}$ & 3.391957 & -2.2325 & 0.5747 \\
$\mathrm{~N}$ & 0.755521 & -0.74868 & 0.083549 & $\mathrm{C}$ & 5.016297 & 1.140691 & -0.42967 \\
$\mathrm{C}$ & -1.11351 & 0.686579 & 0.780481 & $\mathrm{H}$ & 3.102171 & 2.020274 & 0.029263 \\
$\mathrm{O}$ & -1.62712 & 1.049123 & 1.816117 & $\mathrm{C}$ & 5.766596 & -0.03427 & -0.47327 \\
$\mathrm{O}$ & -0.82802 & 1.506171 & -0.23299 & $\mathrm{H}$ & 5.765486 & -2.16316 & -0.13124 \\
$\mathrm{C}$ & -1.19068 & 2.887163 & -0.07172 & $\mathrm{H}$ & 5.467751 & 2.086531 & -0.71454 \\
$\mathrm{H}$ & -2.27238 & 2.94636 & 0.08349 & $\mathrm{H}$ & 6.804711 & -0.0047 & -0.79141 \\
$\mathrm{H}$ & -0.69197 & 3.279033 & 0.819783 & $\mathrm{C}$ & -0.93315 & -1.66154 & 1.665662 \\
$\mathrm{C}$ & -0.76086 & 3.623437 & -1.32167 & $\mathrm{H}$ & -1.99349 & -1.63379 & 1.92817 \\
$\mathrm{H}$ & -1.01686 & 4.683258 & -1.22535 & $\mathrm{H}$ & -0.65605 & -2.68869 & 1.410623 \\
$\mathrm{H}$ & 0.320605 & 3.542161 & -1.46953 & $\mathrm{H}$ & -0.34701 & -1.33336 & 2.527855 \\
$\mathrm{H}$ & -1.26724 & 3.22396 & -2.20567 & $\mathrm{C}$ & -2.59679 & -1.36187 & -1.30819 \\
$\mathrm{C}$ & -1.35057 & -1.32751 & -0.80997 & $\mathrm{Br}$ & -3.9443 & -0.38799 & -0.10328 \\
\hline
\end{tabular}

Table S6. Electronic energies (E), zero-point energies (ZPE), enthalpies (H), and Gibbs free energies (G) (in Hartree, Ha) of the compound calculated at the wb97xd, 6-31+G(d) level of theory. Relative Gibbs free energy is shown in kcal mol ${ }^{-1}$.

\begin{tabular}{cccccc}
\hline Compound & $\mathrm{E}$ & $\mathrm{ZPE}$ & $\mathrm{H}$ & $\mathrm{G}$ & $\Delta G^{a, b}$ \\
\hline $\mathbf{8}(\boldsymbol{Z})$ & -3471.912413 & -3471.610043 & -3471.587418 & -3471.665596 & 0.00 \\
$\mathbf{9}$ & -3471.963809 & -3471.658299 & -3471.637236 & -3471.708590 & -26.98 \\
\hline
\end{tabular}

${ }^{a} 1 \mathrm{Ha}=627.509391 \mathrm{kcal} \mathrm{mol}^{-1} \cdot{ }^{b}$ Relative energy Gibbs free energy between $Z$-enolate 8 and intermediate 9.

\subsection{Procedure for the calculation of $E$-enolate 8 and intermediate 9}

Density functional theory (DFT) calculations were carried out at the wb97xd level of theory with the 6-31+G(d) basis set in Gaussian 09. Optimization and frequency were performed to calculate the Gibbs free energy (reported at $298.15 \mathrm{~K}$ and $1 \mathrm{~atm})$. molecules were modeled in the solvent phase (DMF, polarizable continuum model (PCM)).

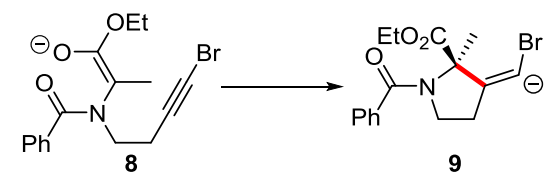

Table S7. Cartesian coordinates of the $E$-enolate 8

\begin{tabular}{lllllllll}
\hline Atom & $\mathrm{X}$ & $\mathrm{Y}$ & $\mathrm{Z}$ & Atom & $\mathrm{X}$ & $\mathrm{Y}$ & $\mathrm{Z}$ \\
\hline
\end{tabular}




\begin{tabular}{cccccccc}
\hline $\mathrm{C}$ & -0.18359 & 0.104076 & -0.18576 & $\mathrm{O}$ & -3.59597 & 1.188084 & 0.335433 \\
$\mathrm{C}$ & 0.706671 & -0.20463 & 1.028481 & $\mathrm{C}$ & -1.68777 & 2.539535 & -0.03077 \\
$\mathrm{C}$ & -2.15765 & -1.18399 & 0.521643 & $\mathrm{C}$ & -0.88981 & 3.098551 & 0.970692 \\
$\mathrm{H}$ & 0.09199 & 1.066848 & -0.61931 & $\mathrm{C}$ & -1.91328 & 3.252687 & -1.21041 \\
$\mathrm{H}$ & -0.0416 & -0.66393 & -0.9516 & $\mathrm{C}$ & -0.3117 & 4.353791 & 0.788197 \\
$\mathrm{H}$ & 0.38835 & -1.15772 & 1.466375 & $\mathrm{H}$ & -0.72303 & 2.5556 & 1.897425 \\
$\mathrm{H}$ & 0.576335 & 0.566089 & 1.79693 & $\mathrm{C}$ & -1.32775 & 4.503453 & -1.3962 \\
$\mathrm{~N}$ & -1.59838 & 0.096391 & 0.174267 & $\mathrm{H}$ & -2.5411 & 2.823288 & -1.98658 \\
$\mathrm{C}$ & -2.25551 & -2.1151 & -0.48636 & $\mathrm{C}$ & -0.52436 & 5.055573 & -0.39816 \\
$\mathrm{O}$ & -1.91916 & -1.99135 & -1.70122 & $\mathrm{H}$ & 0.306616 & 4.781589 & 1.572217 \\
$\mathrm{O}$ & -2.81313 & -3.34143 & -0.0785 & $\mathrm{H}$ & -1.49787 & 5.045879 & -2.32184 \\
$\mathrm{C}$ & -2.24674 & -4.49603 & -0.6851 & $\mathrm{H}$ & -0.06761 & 6.030317 & -0.54332 \\
$\mathrm{H}$ & -2.92087 & -5.31629 & -0.42067 & $\mathrm{C}$ & -2.56014 & -1.36746 & 1.95741 \\
$\mathrm{H}$ & -2.24613 & -4.38448 & -1.77439 & $\mathrm{H}$ & -2.76818 & -2.42105 & 2.162355 \\
$\mathrm{C}$ & -0.83852 & -4.77511 & -0.17661 & $\mathrm{H}$ & -1.76332 & -1.04374 & 2.644861 \\
$\mathrm{H}$ & -0.4445 & -5.69072 & -0.63278 & $\mathrm{H}$ & -3.45936 & -0.79152 & 2.226877 \\
$\mathrm{H}$ & -0.16482 & -3.94969 & -0.43126 & $\mathrm{C}$ & 2.117432 & -0.28717 & 0.656086 \\
$\mathrm{H}$ & -0.836 & -4.90219 & 0.911594 & $\mathrm{C}$ & 3.277045 & -0.35474 & 0.326568 \\
$\mathrm{C}$ & -2.36671 & 1.208307 & 0.168416 & $\mathrm{Br}$ & 4.997145 & -0.45874 & -0.16432 \\
\hline
\end{tabular}

Table S8. Cartesian coordinates of the intermediate 9

\begin{tabular}{cccccccc}
\hline Atom & $\mathrm{X}$ & $\mathrm{Y}$ & $\mathrm{Z}$ & Atom & $\mathrm{X}$ & $\mathrm{Y}$ & $\mathrm{Z}$ \\
\hline $\mathrm{C}$ & 1.354899 & -1.78684 & -0.46268 & $\mathrm{C}$ & 1.775846 & 0.527592 & 0.377812 \\
$\mathrm{C}$ & 0.194127 & -2.71765 & -0.15244 & $\mathrm{O}$ & 1.347583 & 1.639493 & 0.710927 \\
$\mathrm{C}$ & -0.48972 & -0.4452 & 0.454528 & $\mathrm{C}$ & 3.244111 & 0.332039 & 0.125153 \\
$\mathrm{H}$ & 2.31116 & -2.16066 & -0.09617 & $\mathrm{C}$ & 4.016094 & -0.47527 & 0.964099 \\
$\mathrm{H}$ & 1.440392 & -1.59618 & -1.53967 & $\mathrm{C}$ & 3.850646 & 1.022262 & -0.92592 \\
$\mathrm{H}$ & 0.363563 & -3.19518 & 0.822719 & $\mathrm{C}$ & 5.387527 & -0.59284 & 0.750442 \\
$\mathrm{H}$ & 0.078104 & -3.5055 & -0.89952 & $\mathrm{H}$ & 3.544923 & -1.00735 & 1.786849 \\
$\mathrm{~N}$ & 0.972571 & -0.54744 & 0.232296 & $\mathrm{C}$ & 5.220164 & 0.890502 & -1.148 \\
$\mathrm{C}$ & -0.99764 & 0.74717 & -0.37989 & $\mathrm{H}$ & 3.249183 & 1.657056 & -1.57082 \\
$\mathrm{O}$ & -0.75269 & 0.876476 & -1.56469 & $\mathrm{C}$ & 5.990205 & 0.084146 & -0.31019 \\
$\mathrm{O}$ & -1.72175 & 1.60698 & 0.322445 & $\mathrm{H}$ & 5.985302 & -1.21571 & 1.409383
\end{tabular}




\begin{tabular}{llllllll}
$\mathrm{C}$ & -2.32716 & 2.69309 & -0.3946 & $\mathrm{H}$ & 5.685951 & 1.418863 & -1.97476 \\
$\mathrm{H}$ & -1.53647 & 3.35429 & -0.76234 & $\mathrm{H}$ & 7.058022 & -0.0157 & -0.4816 \\
$\mathrm{H}$ & -2.86784 & 2.282946 & -1.25309 & $\mathrm{C}$ & -0.79891 & -0.31757 & 1.950782 \\
$\mathrm{C}$ & -3.25836 & 3.399513 & 0.566537 & $\mathrm{H}$ & -1.87801 & -0.25154 & 2.103841 \\
$\mathrm{H}$ & -3.75949 & 4.226116 & 0.052872 & $\mathrm{H}$ & -0.42168 & -1.20792 & 2.463026 \\
$\mathrm{H}$ & -4.02061 & 2.707064 & 0.937404 & $\mathrm{H}$ & -0.32585 & 0.568324 & 2.381134 \\
$\mathrm{H}$ & -2.7084 & 3.806486 & 1.420713 & $\mathrm{C}$ & -2.2125 & -2.27808 & -0.42721 \\
$\mathrm{C}$ & -1.00856 & -1.78859 & -0.09081 & $\mathrm{Br}$ & -3.70389 & -0.8795 & -0.21603 \\
\hline
\end{tabular}

Table S9. Electronic energies (E), zero-point energies (ZPE), enthalpies (H), and Gibbs free energies (G) (in Hartree, $\mathrm{Ha}$ ) of the compound calculated at the wb97xd, 6-31+G(d) level of theory. Relative Gibbs free energy is shown in kcal $\mathrm{mol}^{-1}$.

\begin{tabular}{cccccc}
\hline Compound & E & ZPE & H & G & $\Delta G^{a, b}$ \\
\hline $\mathbf{8}(\boldsymbol{E})$ & -3471.913009 & -3471.609919 & -3471.587626 & -3471.665240 & 0.00 \\
$\mathbf{9}$ & -3471.963809 & -3471.658299 & -3471.637236 & -3471.708590 & -27.20 \\
\hline
\end{tabular}

${ }^{a} 1 \mathrm{Ha}=627.509391 \mathrm{kcal} \mathrm{mol}^{-1}$. ${ }^{b}$ Relative energy Gibbs free energy between $E$-enolate 8 and intermediate 9.

\subsection{Procedure for the calculation of $E$-enolate $X$ and intermediate $Y$}

Density functional theory (DFT) calculations were carried out at the wb97xd level of theory with the 6-31+G(d) basis set in Gaussian 09. Optimization and frequency were performed to calculate the Gibbs free energy (reported at $298.15 \mathrm{~K}$ and $1 \mathrm{~atm})$. molecules were modeled in the solvent phase (DMF, polarizable continuum model (PCM)).

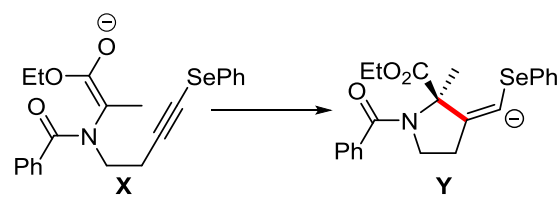

Table S10. Cartesian coordinates of the Z-enolate $\mathbf{X}$

\begin{tabular}{cccccccc}
\hline Atom & $\mathrm{X}$ & $\mathrm{Y}$ & $\mathrm{Z}$ & Atom & $\mathrm{X}$ & $\mathrm{Y}$ & $\mathrm{Z}$ \\
\hline $\mathrm{C}$ & -1.55467 & -0.25238 & 0.173705 & $\mathrm{C}$ & -5.92424 & -1.69901 & 1.472752 \\
$\mathrm{C}$ & -0.91076 & -0.77264 & -1.12714 & $\mathrm{H}$ & -5.11757 & 0.151513 & 2.228003 \\
$\mathrm{C}$ & -1.57132 & 2.12306 & -0.44892 & $\mathrm{C}$ & -5.9872 & -2.57631 & 0.389576 \\
$\mathrm{H}$ & -2.17824 & -1.02266 & 0.630458 & $\mathrm{H}$ & -5.33618 & -2.96108 & -1.62928 \\
$\mathrm{H}$ & -0.76857 & 0.01085 & 0.886105 & $\mathrm{H}$ & -6.47249 & -1.91929 & 2.384282 \\
$\mathrm{H}$ & -0.69564 & 0.086135 & -1.77353 & $\mathrm{H}$ & -6.58634 & -3.47998 & 0.454788 \\
$\mathrm{H}$ & -1.59835 & -1.42714 & -1.67471 & $\mathrm{C}$ & -1.89592 & 2.674866 & -1.80871
\end{tabular}




\begin{tabular}{cccccccc}
$\mathrm{N}$ & -2.33079 & 0.96792 & -0.04577 & $\mathrm{H}$ & -1.19428 & 3.46929 & -2.0738 \\
$\mathrm{C}$ & -0.55559 & 2.53713 & 0.381354 & $\mathrm{H}$ & -1.83951 & 1.901484 & -2.59238 \\
$\mathrm{O}$ & -0.24792 & 2.09439 & 1.528421 & $\mathrm{H}$ & -2.91236 & 3.092359 & -1.85569 \\
$\mathrm{O}$ & 0.231662 & 3.582701 & -0.14147 & $\mathrm{C}$ & 0.347795 & -1.46623 & -0.86504 \\
$\mathrm{C}$ & 1.633658 & 3.341902 & -0.06201 & $\mathrm{C}$ & 1.416679 & -1.96827 & -0.59136 \\
$\mathrm{H}$ & 2.101548 & 4.27463 & -0.39131 & $\mathrm{Se}$ & 3.031108 & -2.69517 & -0.15309 \\
$\mathrm{H}$ & 1.924313 & 3.159087 & 0.979005 & $\mathrm{C}$ & 3.953046 & -1.05001 & 0.248218 \\
$\mathrm{C}$ & 2.073653 & 2.180232 & -0.94305 & $\mathrm{C}$ & 4.88594 & -0.54889 & -0.65733 \\
$\mathrm{H}$ & 3.162131 & 2.065665 & -0.89771 & $\mathrm{C}$ & 3.710112 & -0.3912 & 1.4533 \\
$\mathrm{H}$ & 1.625781 & 1.239615 & -0.60434 & $\mathrm{C}$ & 5.591862 & 0.613657 & -0.34728 \\
$\mathrm{H}$ & 1.781534 & 2.349258 & -1.98571 & $\mathrm{H}$ & 5.066484 & -1.0618 & -1.59718 \\
$\mathrm{C}$ & -3.67043 & 1.037471 & 0.116883 & $\mathrm{C}$ & 4.400178 & 0.78283 & 1.746685 \\
$\mathrm{O}$ & -4.29864 & 2.108058 & 0.137113 & $\mathrm{H}$ & 2.983664 & -0.78735 & 2.15644 \\
$\mathrm{C}$ & -4.43966 & -0.25304 & 0.225863 & $\mathrm{C}$ & 5.344815 & 1.282928 & 0.849897 \\
$\mathrm{C}$ & -4.50774 & -1.1318 & -0.85868 & $\mathrm{H}$ & 6.323645 & 1.001897 & -1.04959 \\
$\mathrm{C}$ & -5.16091 & -0.53628 & 1.387857 & $\mathrm{H}$ & 4.203675 & 1.30331 & 2.679358 \\
$\mathrm{C}$ & -5.28302 & -2.28713 & -0.77903 & $\mathrm{H}$ & 5.887289 & 2.193876 & 1.085071 \\
$\mathrm{H}$ & -3.96109 & -0.90595 & -1.77065 & & & & \\
\hline
\end{tabular}

Table S11. Cartesian coordinates of the intermediate $\mathbf{Y}$

\begin{tabular}{cccccccc}
\hline Atom & $\mathrm{X}$ & $\mathrm{Y}$ & $\mathrm{Z}$ & Atom & $\mathrm{X}$ & $\mathrm{Y}$ & $\mathrm{Z}$ \\
\hline $\mathrm{C}$ & -2.11491 & -1.85672 & -0.09153 & $\mathrm{H}$ & -4.7231 & -1.16107 & -1.78448 \\
$\mathrm{C}$ & -0.83912 & -2.35241 & -0.75548 & $\mathrm{C}$ & -6.35047 & -0.37483 & 1.644376 \\
$\mathrm{C}$ & -0.77717 & 0.087989 & -0.75275 & $\mathrm{H}$ & -4.5481 & 0.729693 & 2.069844 \\
$\mathrm{H}$ & -3.01448 & -2.35285 & -0.45641 & $\mathrm{C}$ & -7.03849 & -1.14444 & 0.706447 \\
$\mathrm{H}$ & -2.06789 & -1.97 & 0.998976 & $\mathrm{H}$ & -6.99042 & -2.01332 & -1.26612 \\
$\mathrm{H}$ & -1.05286 & -2.59764 & -1.80666 & $\mathrm{H}$ & -6.80287 & -0.15567 & 2.607127 \\
$\mathrm{H}$ & -0.43777 & -3.24791 & -0.27532 & $\mathrm{H}$ & -8.02954 & -1.52457 & 0.936762 \\
$\mathrm{~N}$ & -2.1259 & -0.42612 & -0.43861 & $\mathrm{C}$ & -0.74493 & 0.670202 & -2.17177 \\
$\mathrm{C}$ & -0.39915 & 1.098833 & 0.342173 & $\mathrm{H}$ & 0.261043 & 1.016796 & -2.42228 \\
$\mathrm{O}$ & -0.53974 & 0.882161 & 1.530236 & $\mathrm{H}$ & -1.025 & -0.11959 & -2.87563 \\
$\mathrm{O}$ & 0.140652 & 2.212787 & -0.14222 & $\mathrm{H}$ & -1.44023 & 1.506595 & -2.28015 \\
$\mathrm{C}$ & 0.687715 & 3.138395 & 0.811761 & $\mathrm{C}$ & 1.442776 & -1.34379 & -0.50272 \\
$\mathrm{H}$ & -0.12718 & 3.522525 & 1.432501 & $\mathrm{Se}$ & 2.533218 & 0.267598 & -0.42776 \\
$\mathrm{H}$ & 1.391283 & 2.594405 & 1.449811 & $\mathrm{C}$ & 4.186286 & -0.54386 & 0.090932 \\
$\mathrm{C}$ & 1.374003 & 4.238602 & 0.032521 & $\mathrm{C}$ & 4.290785 & -1.1912 & 1.324852 \\
$\mathrm{H}$ & 1.819918 & 4.955748 & 0.728962 & $\mathrm{C}$ & 5.316689 & -0.45404 & -0.72329 \\
$\mathrm{H}$ & 2.169324 & 3.825229 & -0.59587 & $\mathrm{C}$ & 5.501015 & -1.74385 & 1.737625
\end{tabular}




\begin{tabular}{llllllll}
$\mathrm{H}$ & 0.662415 & 4.772014 & -0.60549 & $\mathrm{H}$ & 3.417408 & -1.26266 & 1.96739 \\
$\mathrm{C}$ & 0.115325 & -1.17299 & -0.64983 & $\mathrm{C}$ & 6.535748 & -0.99314 & -0.30733 \\
$\mathrm{C}$ & -3.14703 & 0.422809 & -0.20373 & $\mathrm{H}$ & 5.24865 & 0.042314 & -1.68807 \\
$\mathrm{O}$ & -3.01228 & 1.653206 & -0.25123 & $\mathrm{C}$ & 6.629971 & -1.64305 & 0.922429 \\
$\mathrm{C}$ & -4.4896 & -0.17075 & 0.116027 & $\mathrm{H}$ & 5.566972 & -2.24583 & 2.699323 \\
$\mathrm{C}$ & -5.17962 & -0.94216 & -0.8224 & $\mathrm{H}$ & 7.407738 & -0.91372 & -0.95101 \\
$\mathrm{C}$ & -5.08211 & 0.11969 & 1.346478 & $\mathrm{H}$ & 7.577235 & -2.06558 & 1.245717 \\
$\mathrm{C}$ & -6.45433 & -1.42189 & -0.52967 & & & & \\
\hline
\end{tabular}

Table S12. Electronic energies (E), zero-point energies (ZPE), enthalpies (H), and Gibbs free energies (G) (in Hartree, $\mathrm{Ha}$ ) of the compound calculated at the wb97xd, 6-31+G(d) level of theory. Relative Gibbs free energy is shown in kcal $\mathrm{mol}^{-1}$.

\begin{tabular}{cccccc}
\hline Compound & E & ZPE & H & G & \multicolumn{1}{c}{$\Delta G^{a, b}$} \\
\hline $\mathbf{X}(\boldsymbol{Z})$ & -3531.19 & -3530.794114 & -3530.766862 & -3530.855443 & 0.00 \\
$\mathbf{Y}$ & -3531.23 & -3530.828096 & -3530.801937 & -3530.885494 & -18.86 \\
\hline
\end{tabular}

${ }^{a} 1 \mathrm{Ha}=627.509391 \mathrm{kcal} \mathrm{mol}^{-1} \cdot{ }^{b}$ Relative energy Gibbs free energy between Z-enolate $\mathbf{X}$ and intermediate $\mathbf{Y}$. 


\section{Chiral HPLC Data}

1. HPLC analysis: Chiralcel OD-H (hexane $/{ }^{i} \mathrm{PrOH}, 95 / 5,0.5 \mathrm{~mL} / \mathrm{min}, 254 \mathrm{~nm}, 25^{\circ} \mathrm{C}$ ); $30.9 \mathrm{~min} ;>99 \%$ ee.

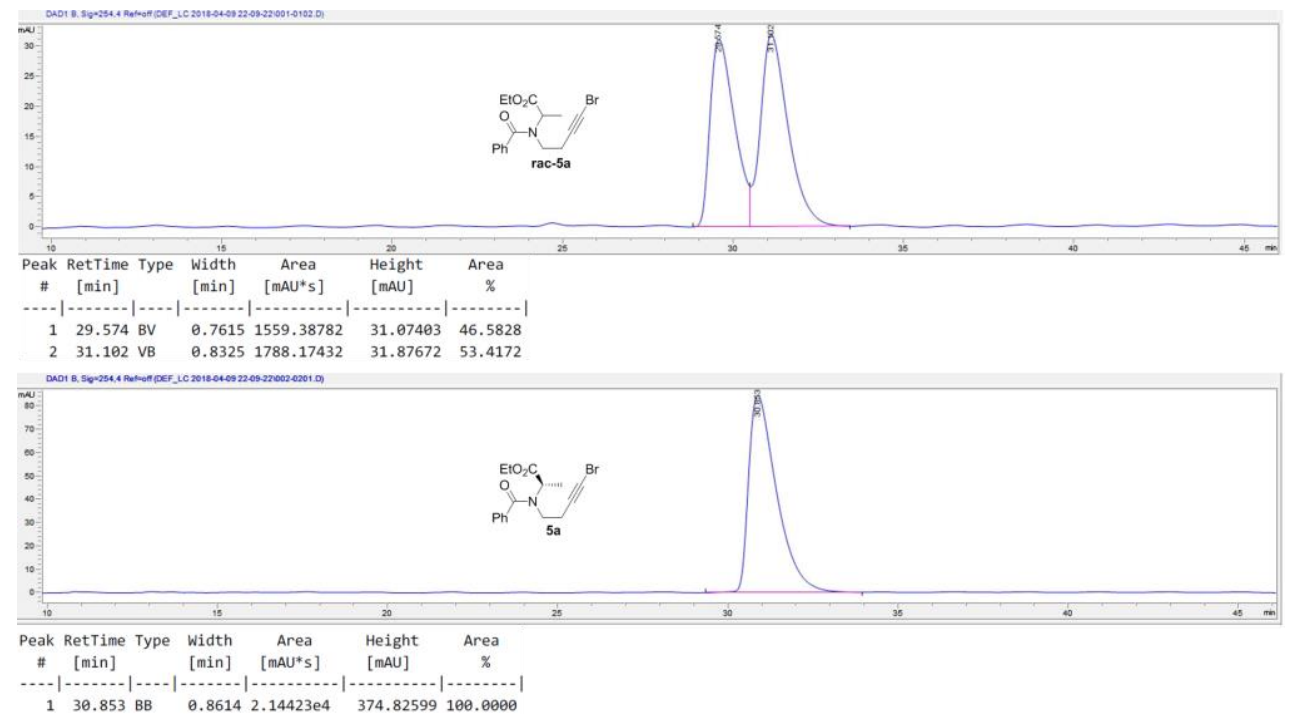

2. HPLC analysis: Chiralcel OD-H (hexane $/{ }^{i} \mathrm{PrOH}, 85 / 15,0.5 \mathrm{~mL} / \mathrm{min}, 254 \mathrm{~nm}, 25^{\circ} \mathrm{C}$ ); 19.4 (minor), 38.7 (major) min; 98\% ee.

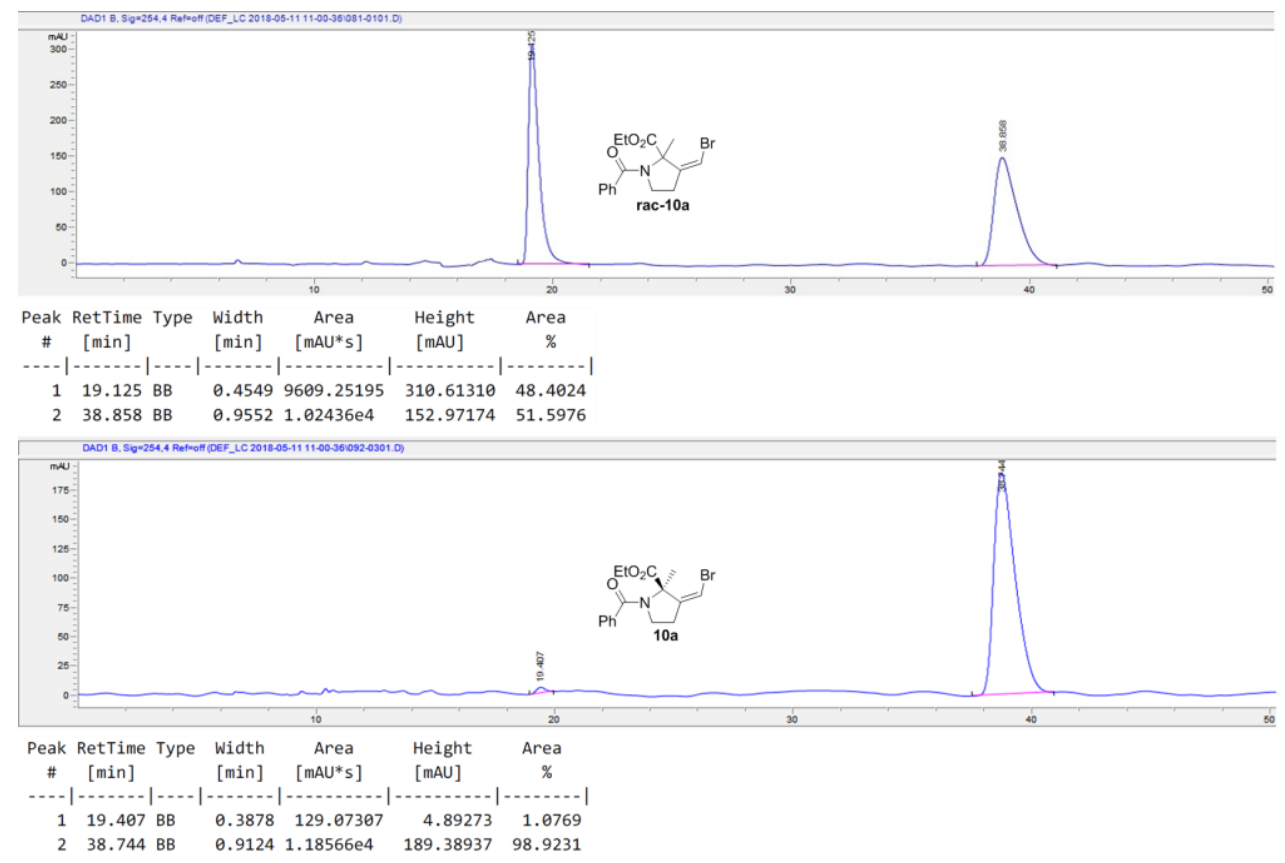

3. HPLC analysis: Chiralcel OD-H (hexane/ ${ }^{i} \mathrm{PrOH}, 85 / 15,0.5 \mathrm{~mL} / \mathrm{min}, 254 \mathrm{~nm}, 25^{\circ} \mathrm{C}$ ); 18.3 (minor), 40.0 (major) $\min ; 94 \%$ ee. 


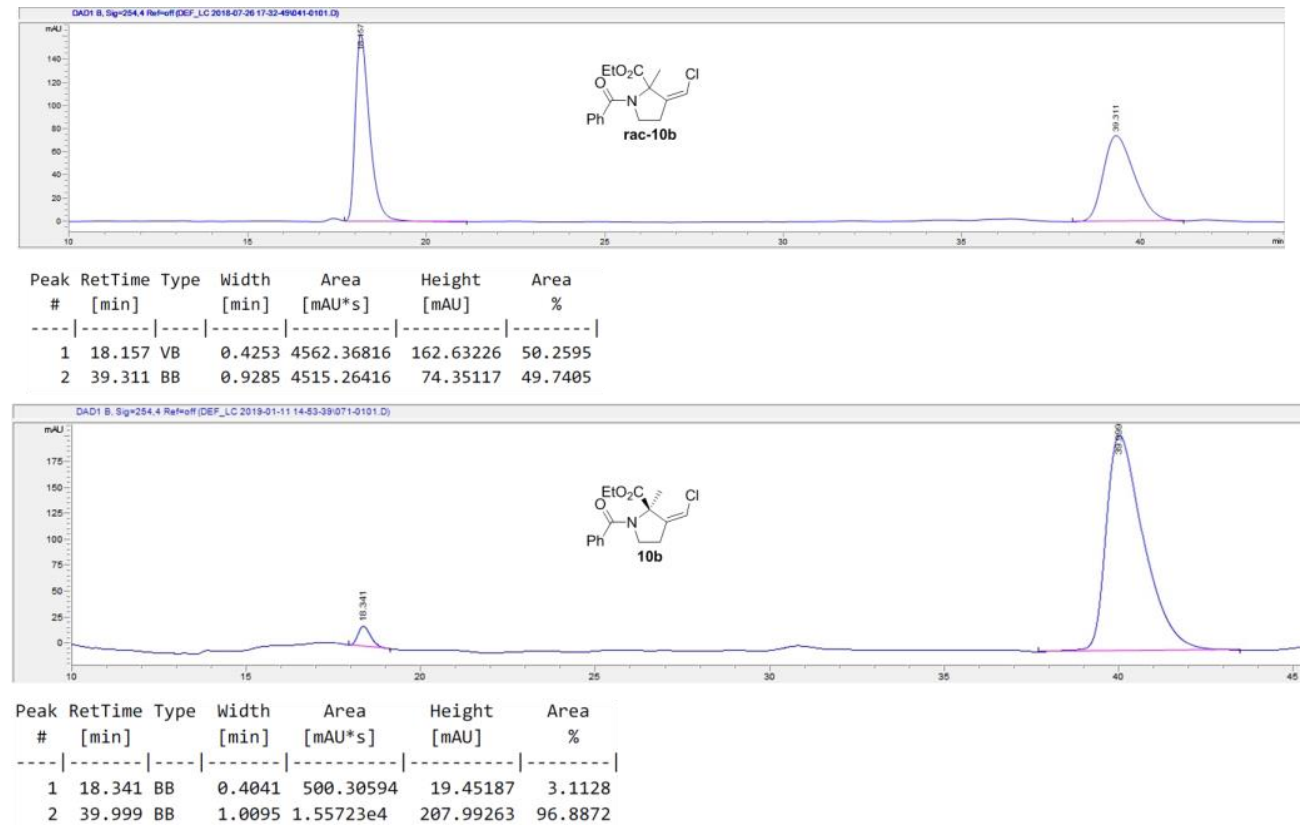

4. HPLC analysis: Chiralcel OD-H (hexane $/{ }^{i} \mathrm{PrOH}, 85 / 15,0.5 \mathrm{~mL} / \mathrm{min}, 254 \mathrm{~nm}, 25^{\circ} \mathrm{C}$ ); 19.7 (minor), 39.1 (major) $\min ; 83 \%$ ee.
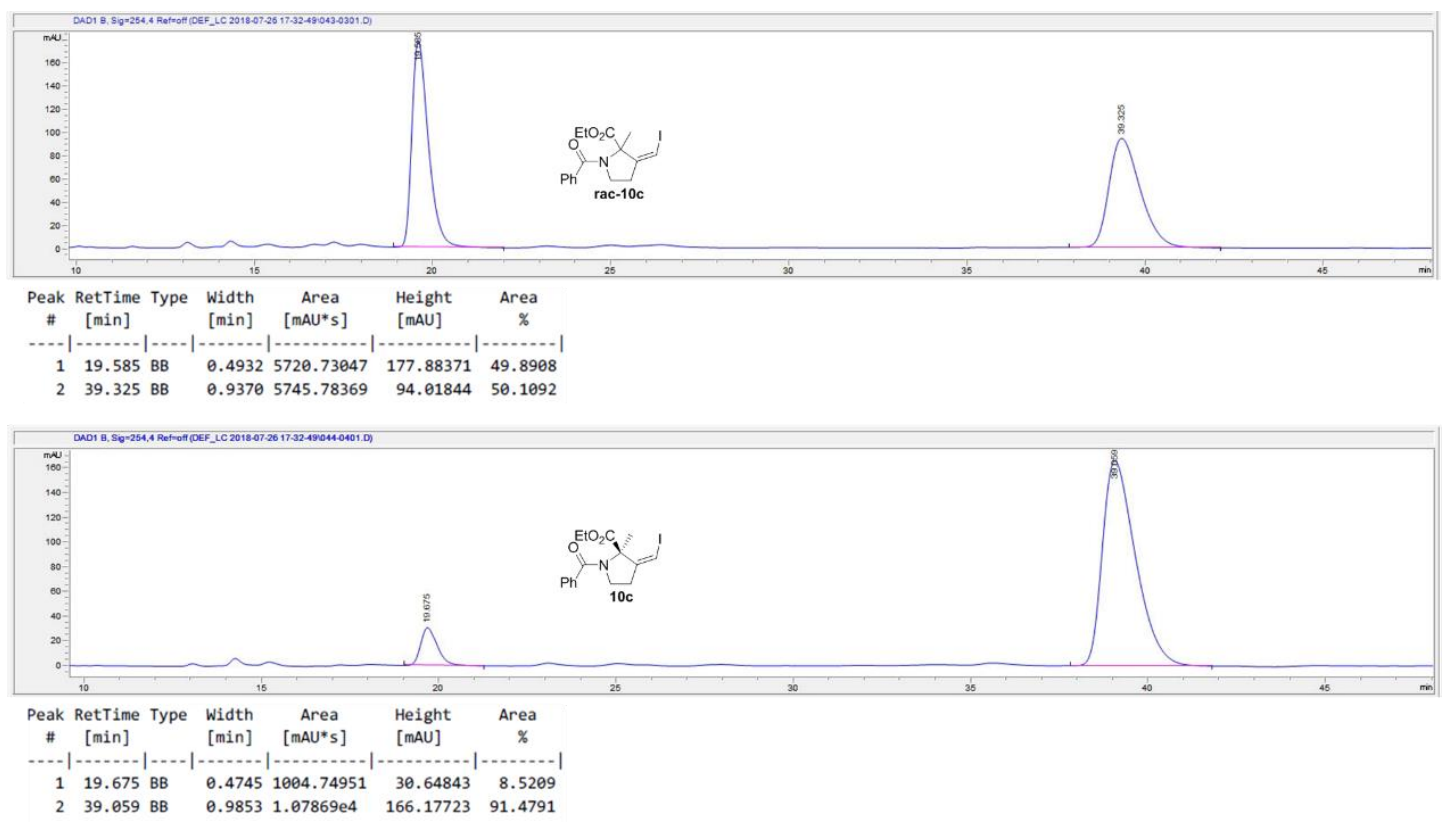

5. HPLC analysis: Chiralcel OD-H (hexane $/{ }^{i} \mathrm{PrOH}, 70 / 30,0.5 \mathrm{~mL} / \mathrm{min}, 254 \mathrm{~nm}, 25^{\circ} \mathrm{C}$ ); 14.3 (minor), 22.9 (major) min; 93\% ee. 


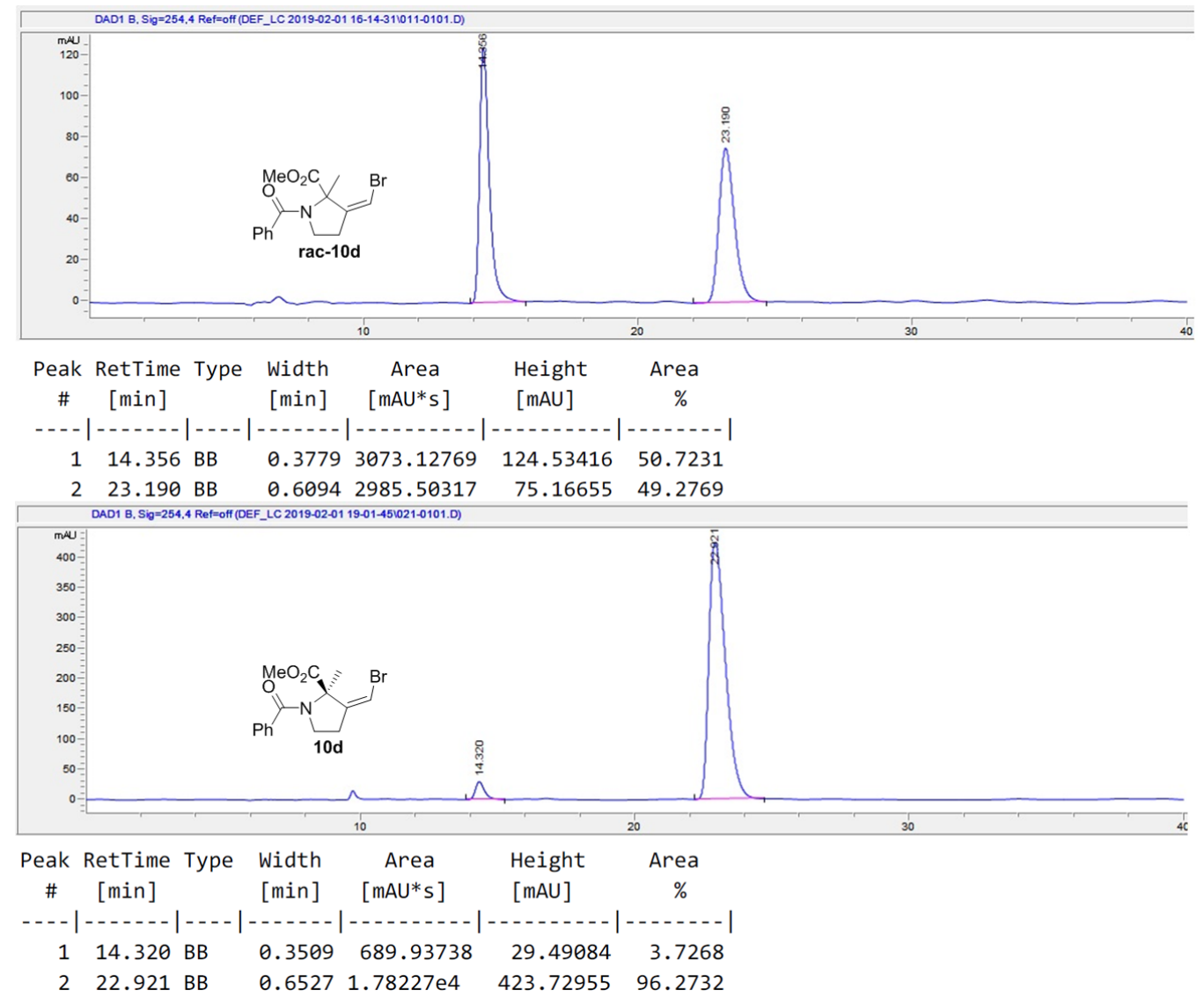

6. HPLC analysis: Chiralcel OD-H (hexane $/{ }^{i} \mathrm{PrOH}, 60 / 40,0.1 \mathrm{~mL} / \mathrm{min}, 254 \mathrm{~nm}, 25^{\circ} \mathrm{C}$ ); 47.3 (minor), 48.7 (major) $\min ; 97 \%$ ee.

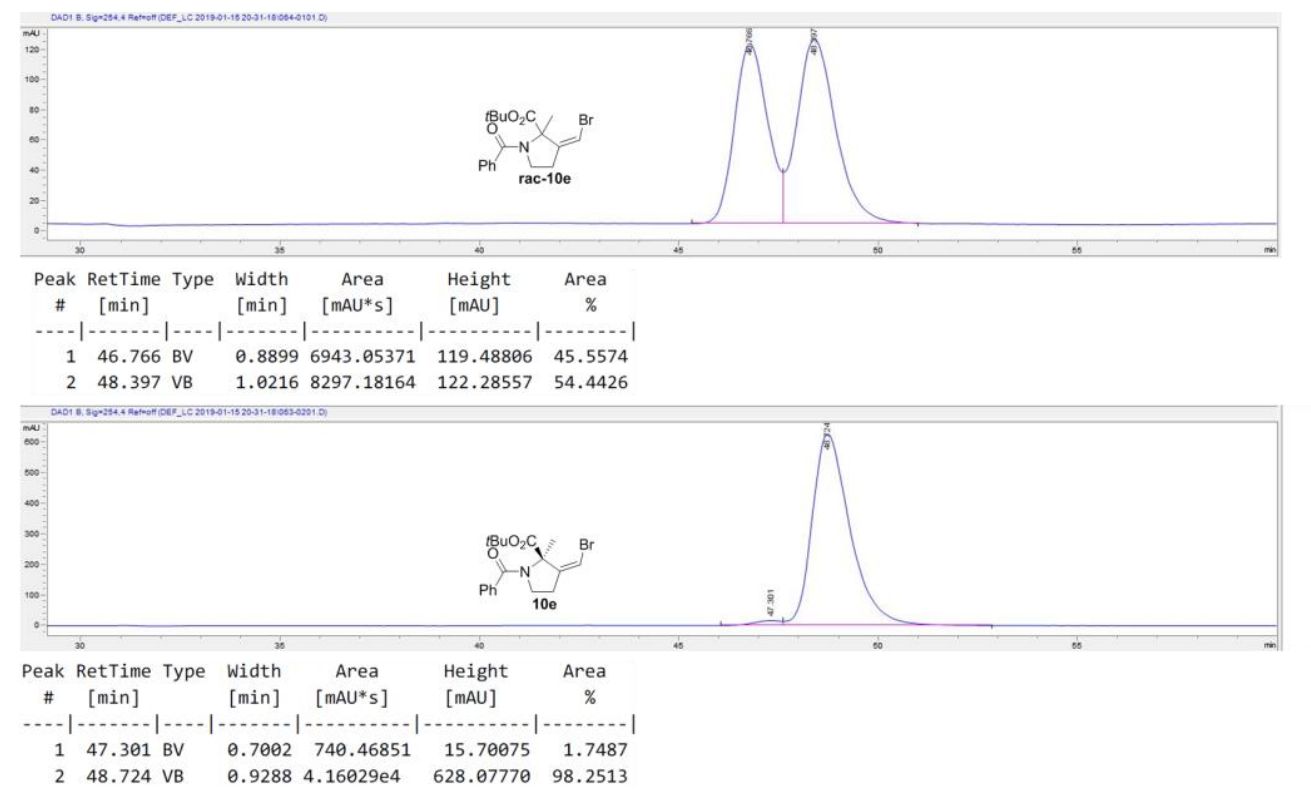


7. HPLC analysis: Because $\mathbf{1 0 f}$ can not be fully separated after trying a few chiral columns and conditions, we transform 10f to 10f' for HPLC analysis. Chiralcel OD-H (hexane/iPrOH, 90/10, 0.5 mL/min, $254 \mathrm{~nm}, 25^{\circ} \mathrm{C}$ ); 26.0 (minor), 59.4 (major) min; 98\% ee.
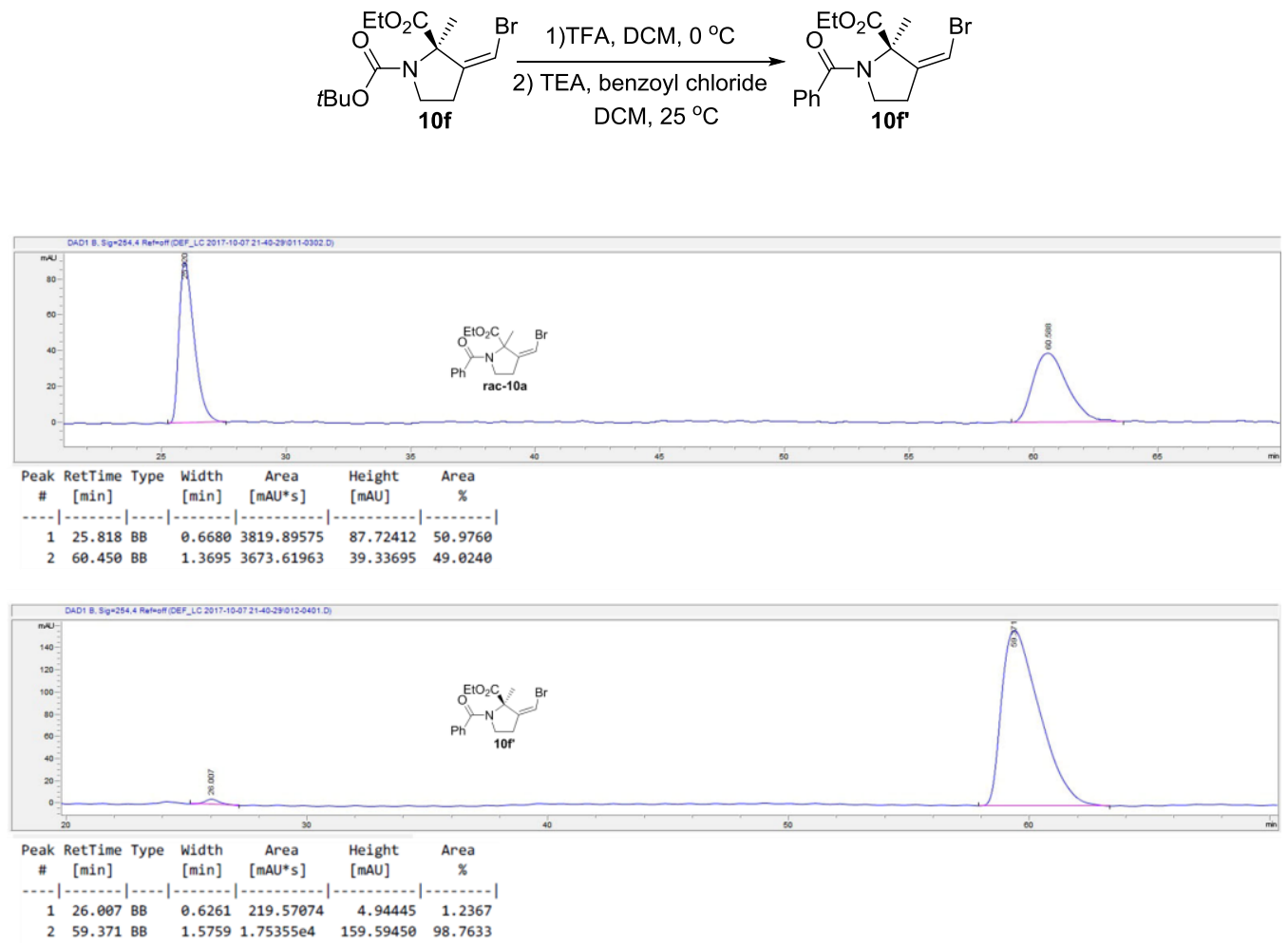

8. HPLC analysis: Chiralcel OD-H (hexane $/{ }^{i} \mathrm{PrOH}, 90 / 10,0.5 \mathrm{~mL} / \mathrm{min}, 210 \mathrm{~nm}, 25^{\circ} \mathrm{C}$ ); 17.4 (minor), 27.1 (major) $\min ; 97 \%$ ee.

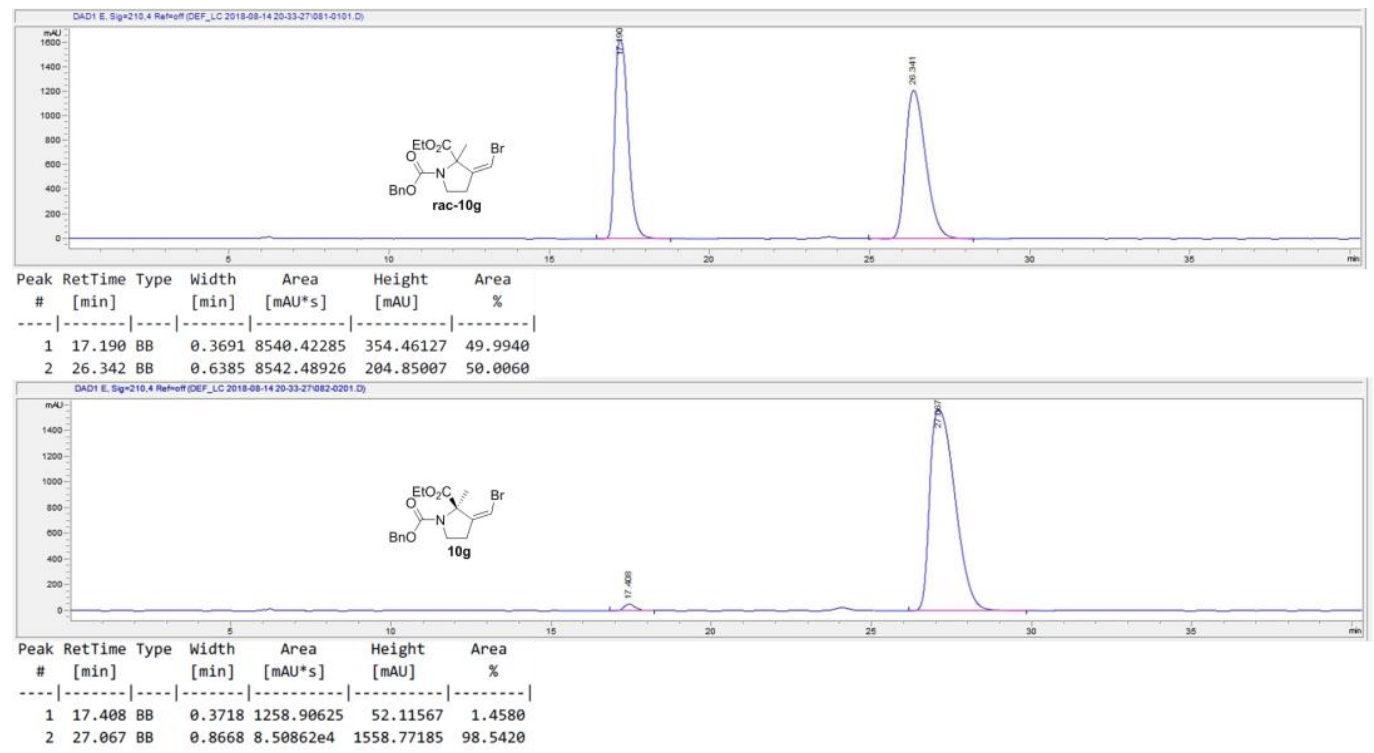


9. HPLC analysis: Because 10h can not be fully separated after trying a few chiral columns and conditions, we transform 10h to 10h' for HPLC analysis. Chiralcel OD-H (hexane $/{ }^{2} \mathrm{PrOH}, 80 / 20,0.5 \mathrm{~mL} / \mathrm{min}, 254 \mathrm{~nm}, 25{ }^{\circ} \mathrm{C}$ ); 11.4 (minor), 52.3 (major) min; 99\% ee.
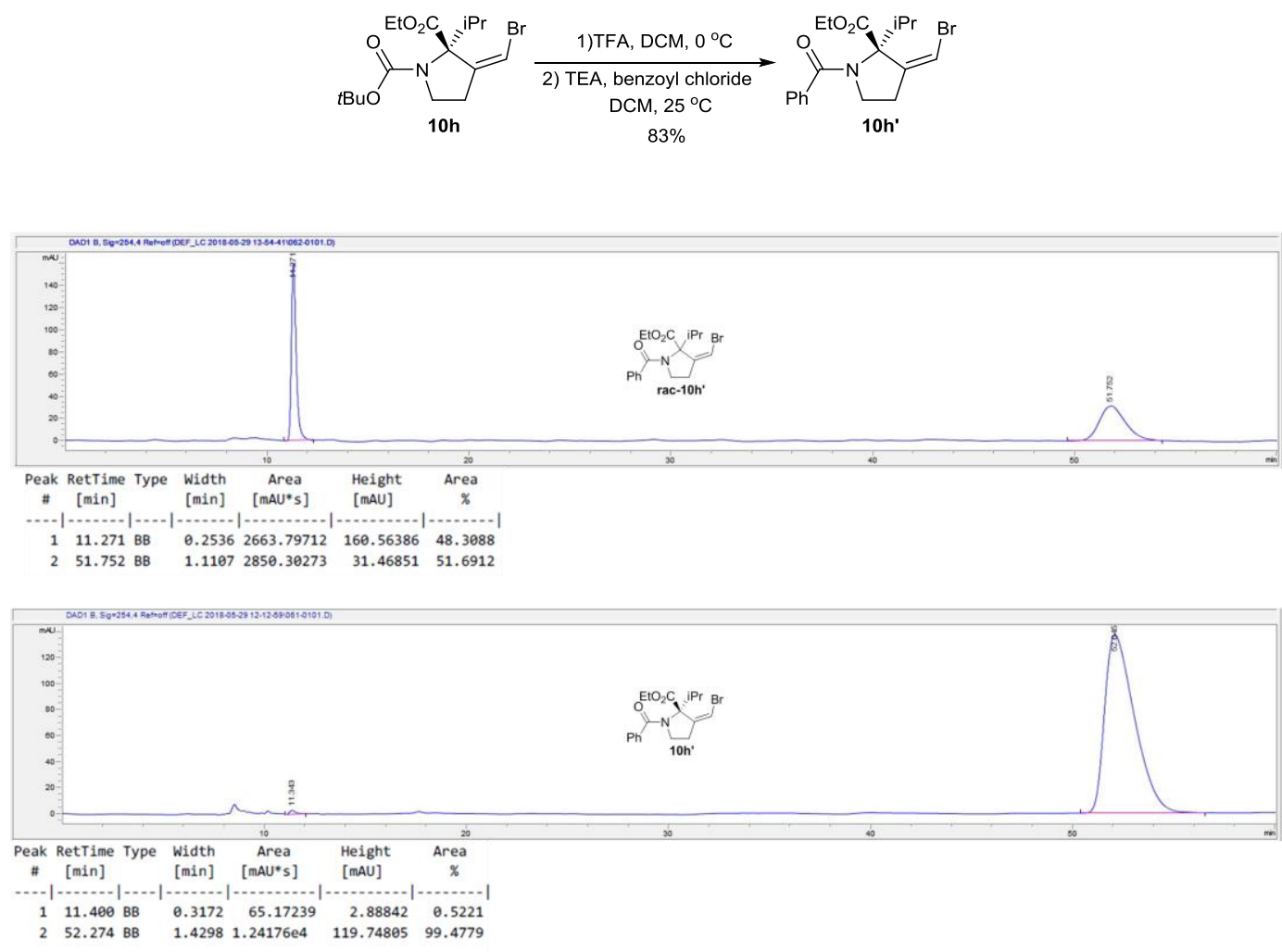

10. HPLC analysis: Chiralpak AD-3R $\left(\mathrm{H}_{2} \mathrm{O} / \mathrm{ACN}, 45 / 55,0.2 \mathrm{~mL} / \mathrm{min}, 210 \mathrm{~nm}, 25^{\circ} \mathrm{C}\right) ; 38.7$ (minor), 41.9 (major) $\min ; 96 \%$ ee.
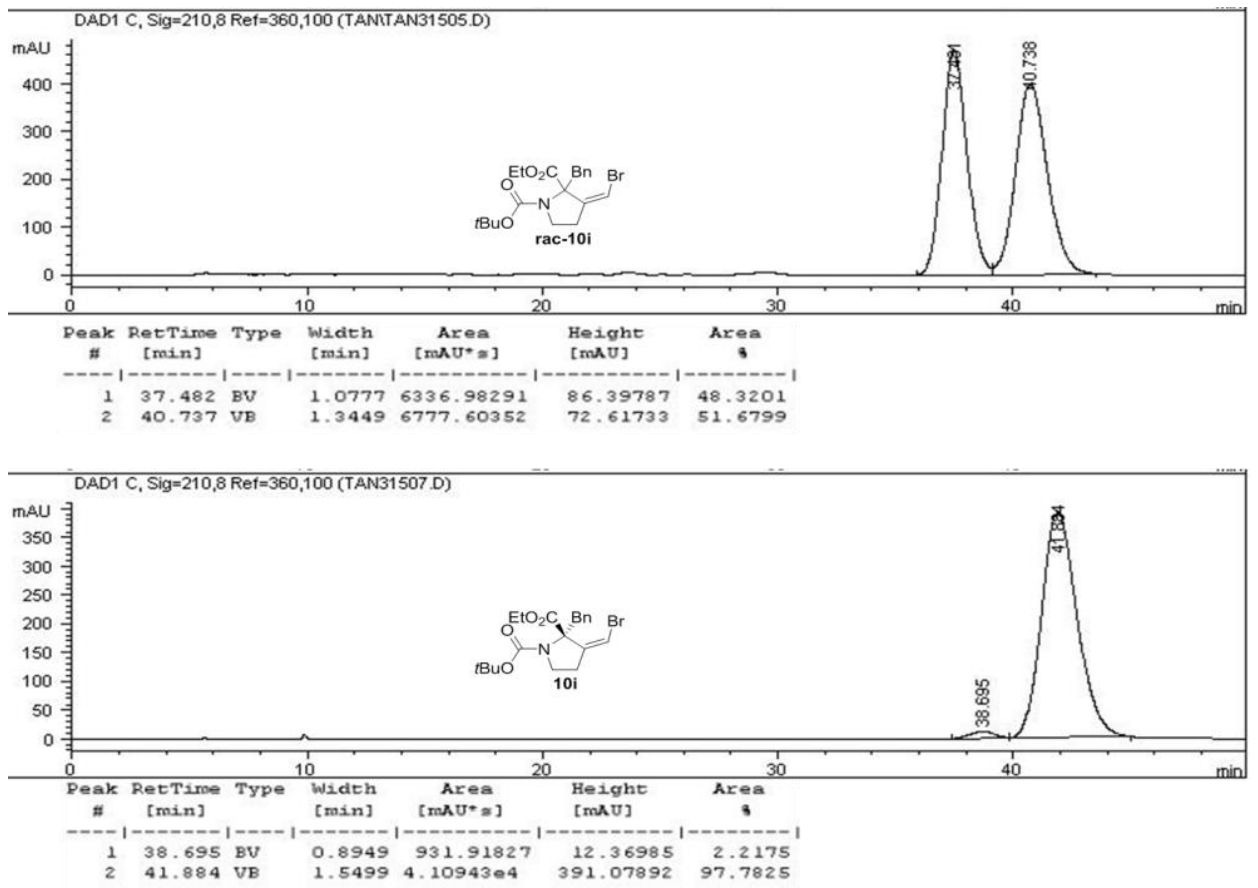
11. HPLC analysis: Chiralcel OD-H (hexane/ $\mathrm{PrOH}, 85 / 15,0.5 \mathrm{~mL} / \mathrm{min}, 225 \mathrm{~nm}, 25^{\circ} \mathrm{C}$ ); 9.7 (minor), 11.0 (major) $\min ; 90 \%$ ee.

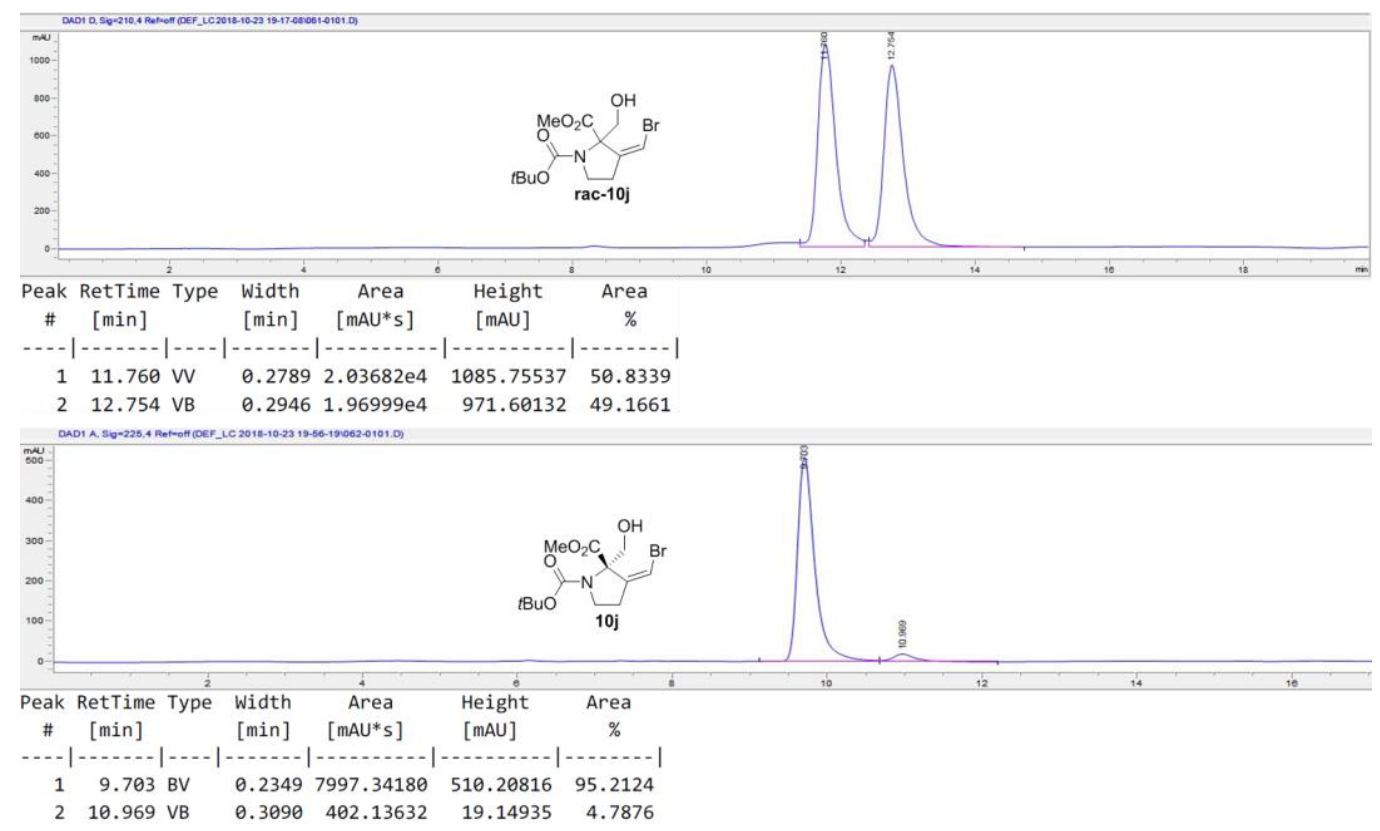

12. HPLC analysis: Chiralcel OD-H (hexane $/{ }^{i} \mathrm{PrOH}, 95 / 5,0.2 \mathrm{~mL} / \mathrm{min}, 225 \mathrm{~nm}, 25^{\circ} \mathrm{C}$ ); 19.0 (minor), 20.1 (major) $\min$; 98\% ee.

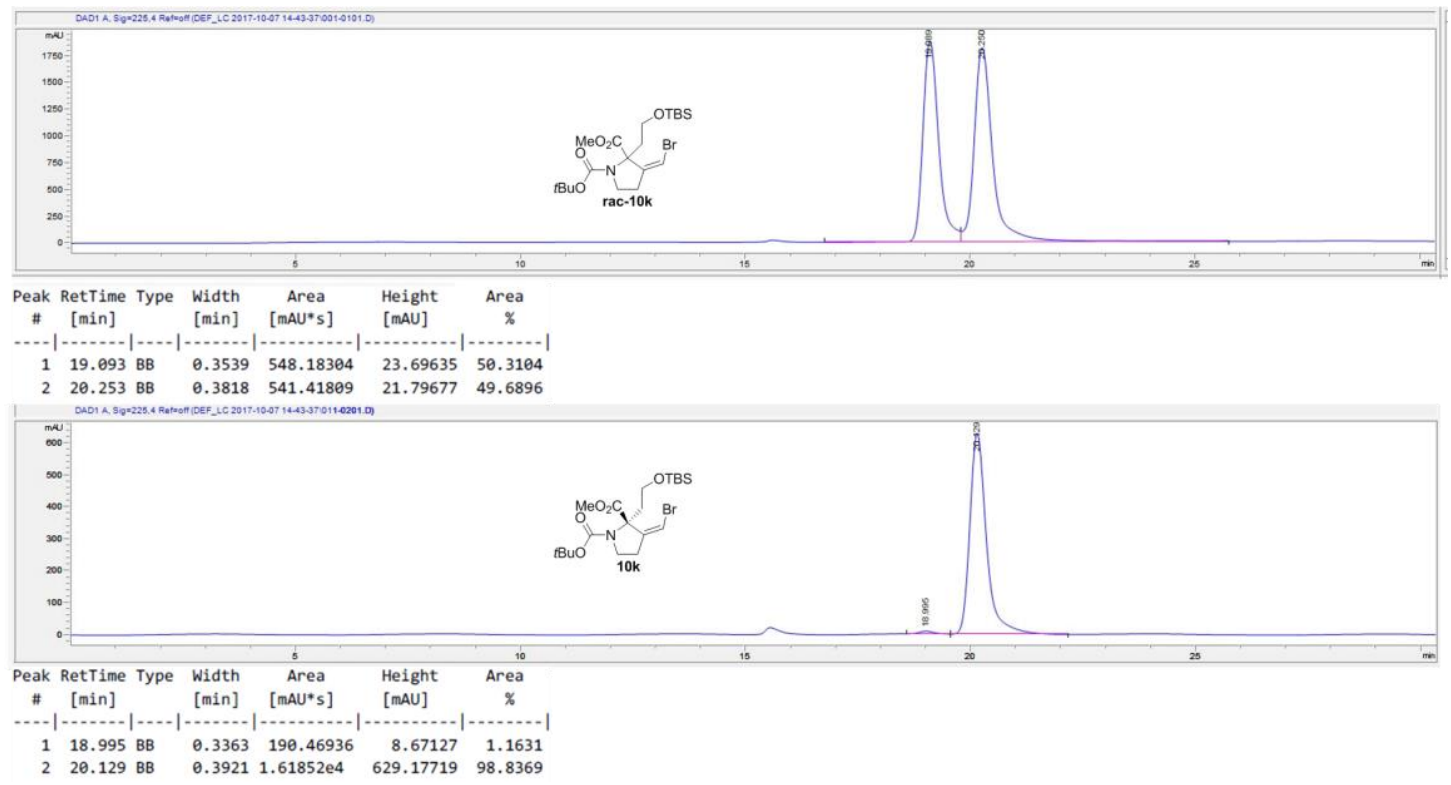

13. HPLC analysis: Chiralpak AD-H (hexane/ $/ \mathrm{PrOH}, 80 / 20,0.3 \mathrm{~mL} / \mathrm{min}, 210 \mathrm{~nm}, 25^{\circ} \mathrm{C}$ ); 16.8 (minor), 18.7 (major) $\min ; 99 \%$ ee. 


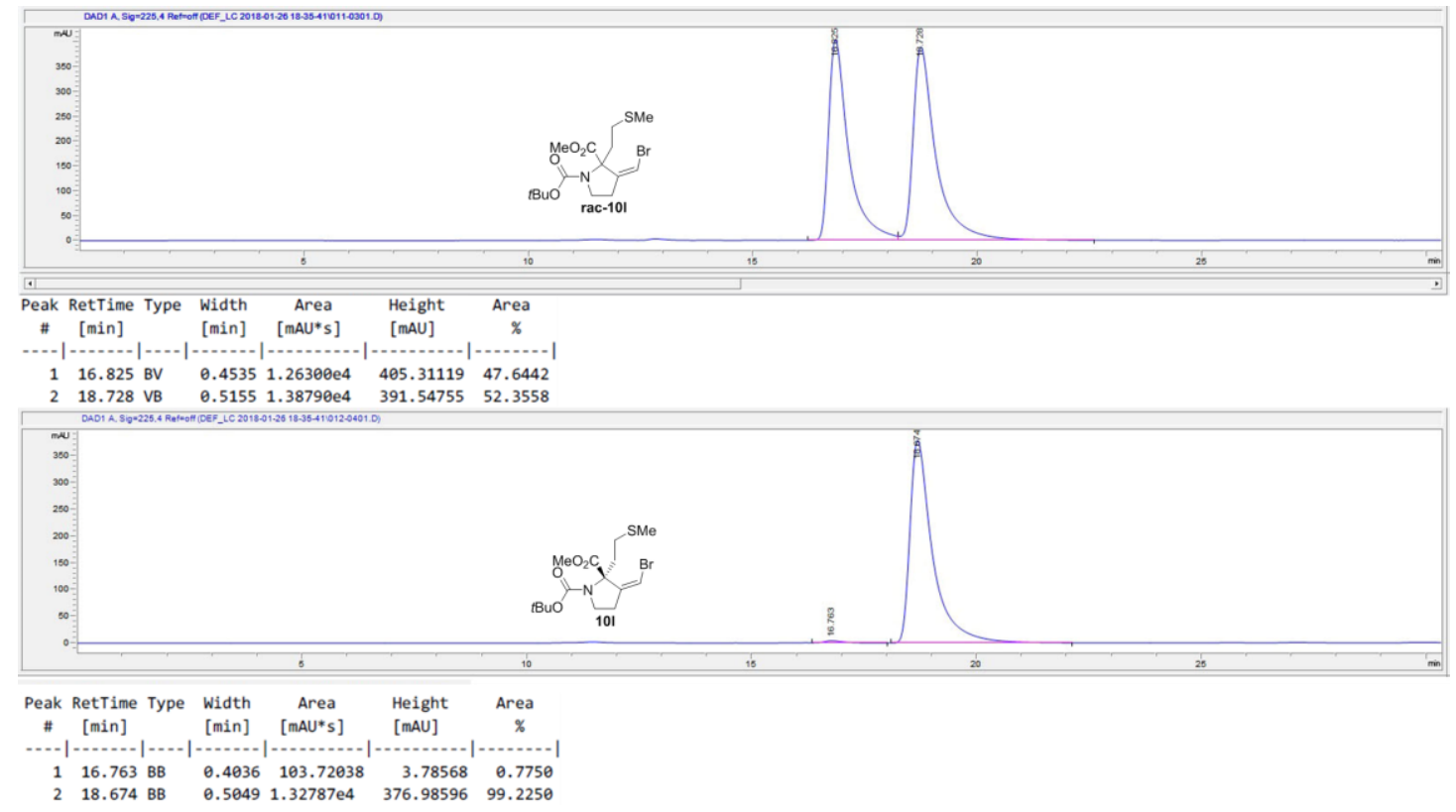

14. HPLC analysis: Because $\mathbf{1 0 m}$ can not be fully separated after trying a few chiral columns and conditions, we transform 10m to 10m' for HPLC analysis. Chiralcel OD-H (hexane $/{ }^{i} \mathrm{PrOH}, 60 / 40,0.5 \mathrm{~mL} / \mathrm{min}, 254 \mathrm{~nm}, 25{ }^{\circ} \mathrm{C}$ ); 25.4 (minor), 14.5 (major) min; 96\% ee.
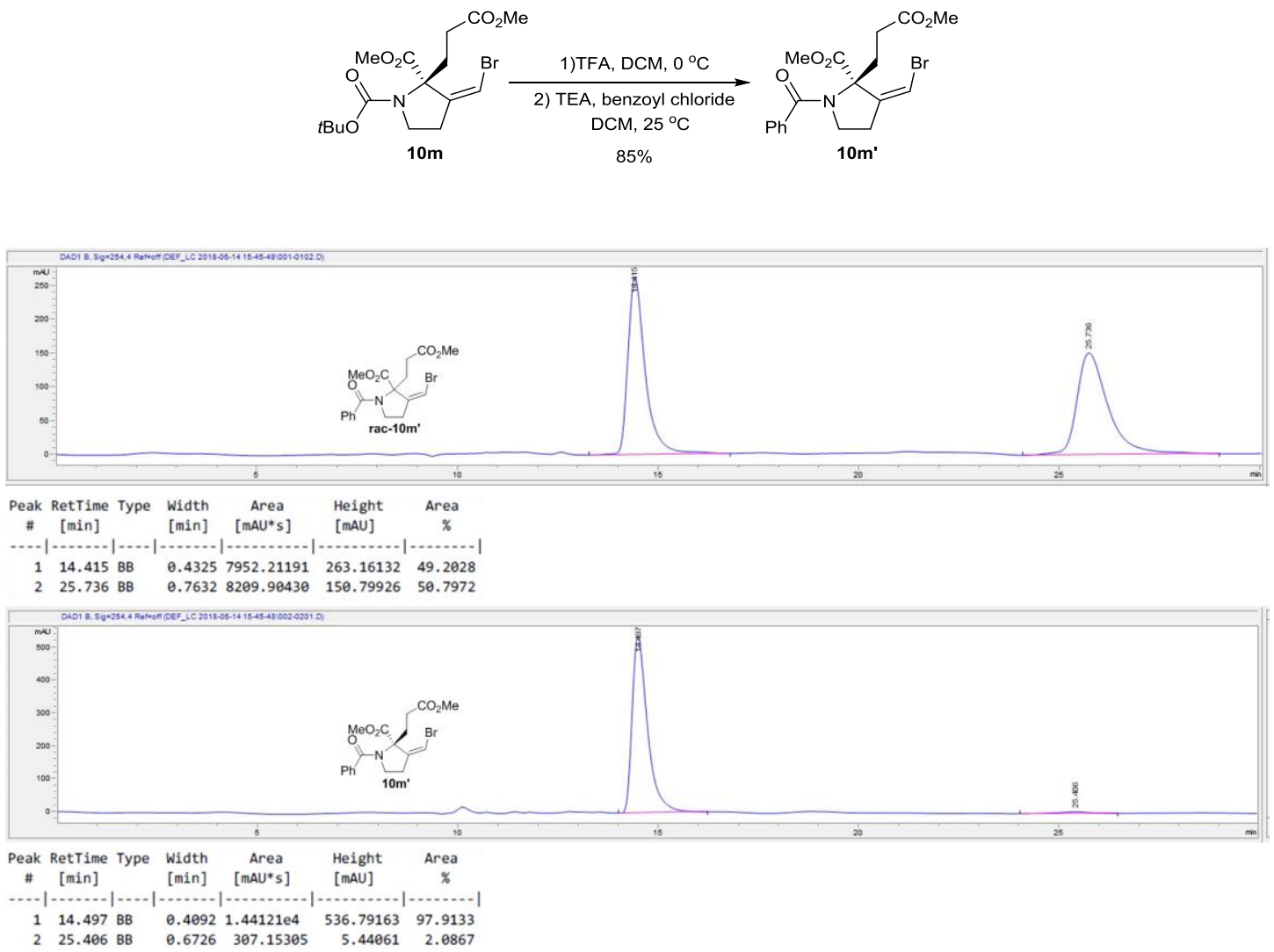
15. HPLC analysis: Chiralcel OD-H (hexane $/{ }^{i} \mathrm{PrOH}, 85 / 15,0.5 \mathrm{~mL} / \mathrm{min}, 254 \mathrm{~nm}, 25^{\circ} \mathrm{C}$ ); 23.4 (minor), 28.3 (major) $\min ; 92 \%$ ee.

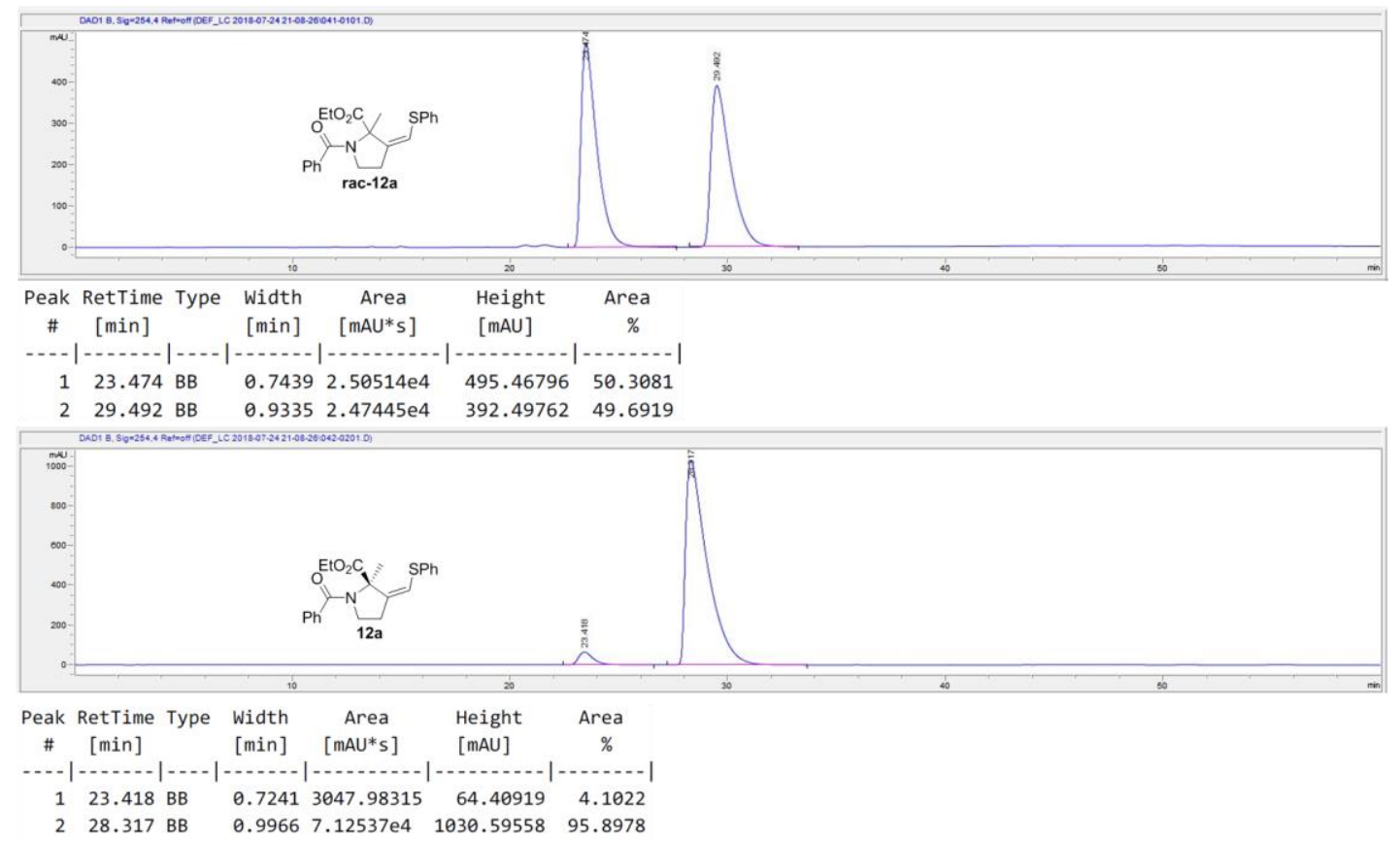

16. HPLC analysis: Chiralcel OD-H (hexane $/{ }^{i} \mathrm{PrOH}, 85 / 15,0.5 \mathrm{~mL} / \mathrm{min}, 254 \mathrm{~nm}, 25^{\circ} \mathrm{C}$ ); 21.5 (minor), 26.4 (major) $\min ; 92 \%$ ee.

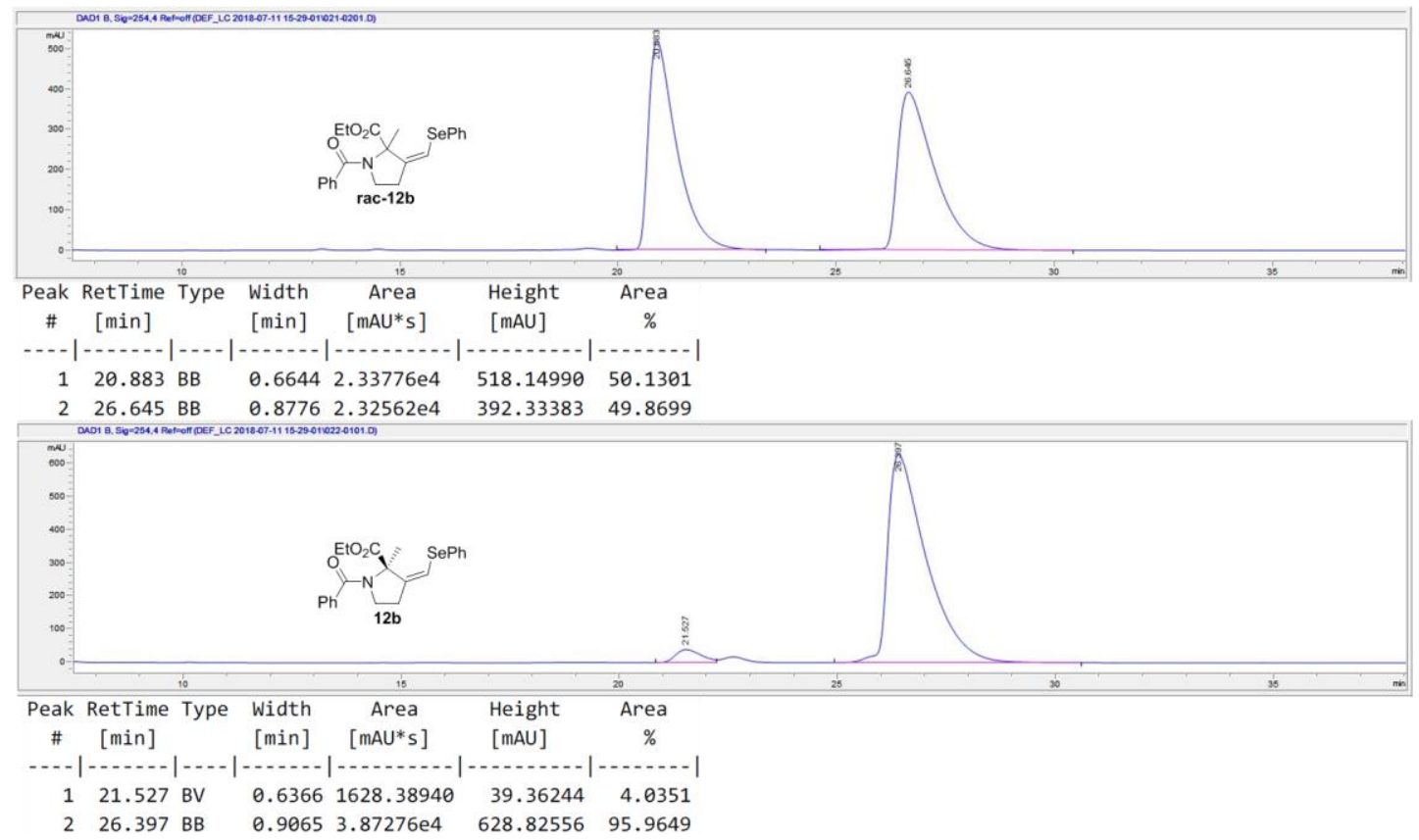

17. HPLC analysis: Because 12c can not be fully separated after trying a few chiral columns and conditions, we transform 12c to 12c' for HPLC analysis. Chiralcel OD-H (hexane $/{ }^{i} \mathrm{PrOH}, 70 / 30,0.5 \mathrm{~mL} / \mathrm{min}, 254 \mathrm{~nm}, 25{ }^{\circ} \mathrm{C}$ ); 12.4 (minor), 27.0 (major) $\min$; 93\% ee. 

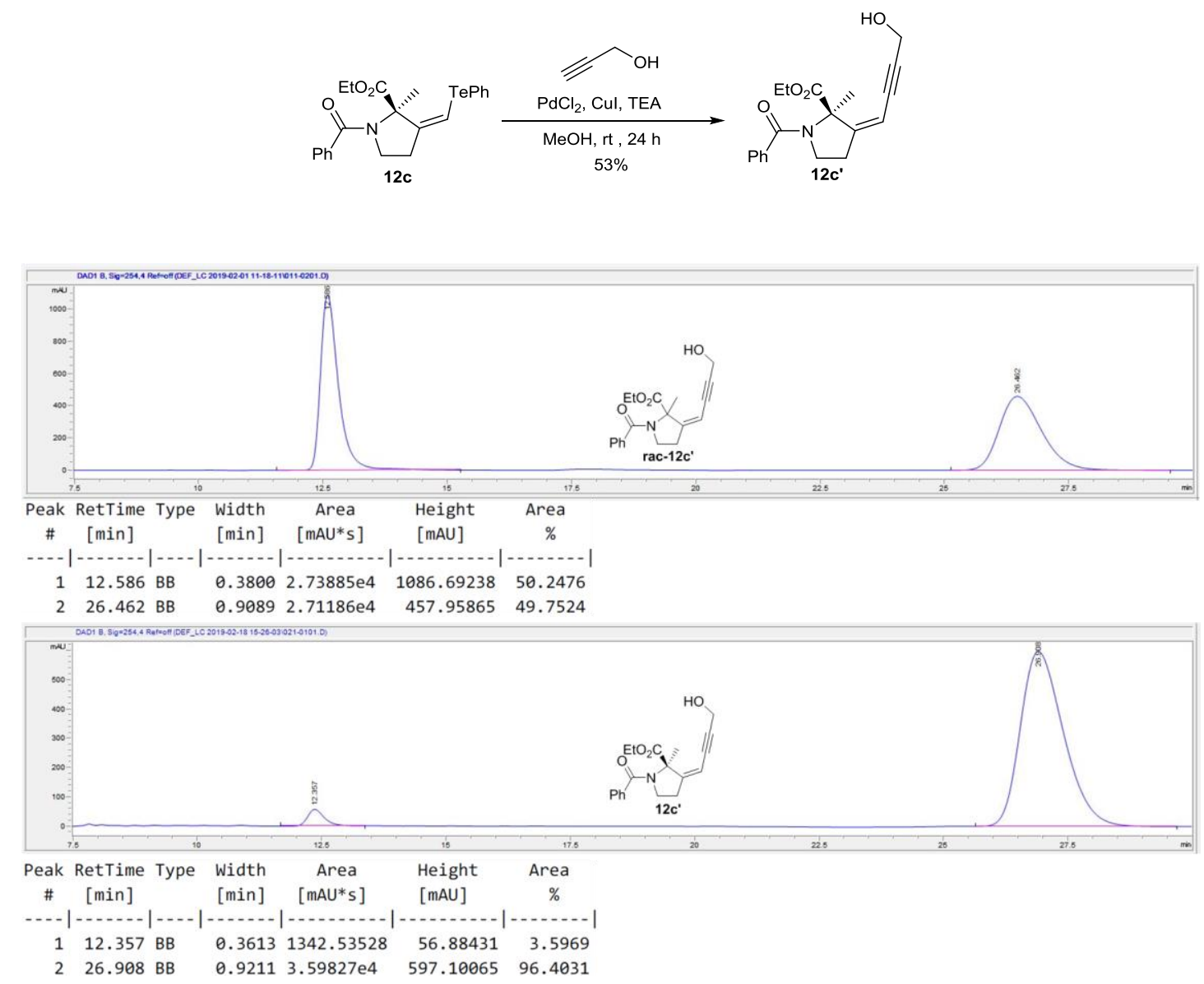

18. HPLC analysis: Chiralcel OD-H (hexane $/{ }^{i} \mathrm{PrOH}, 80 / 20,0.3 \mathrm{~mL} / \mathrm{min}, 254 \mathrm{~nm}, 25^{\circ} \mathrm{C}$ ); 15.4 (minor), 14.2 (major) $\min ; 96 \%$ ee.

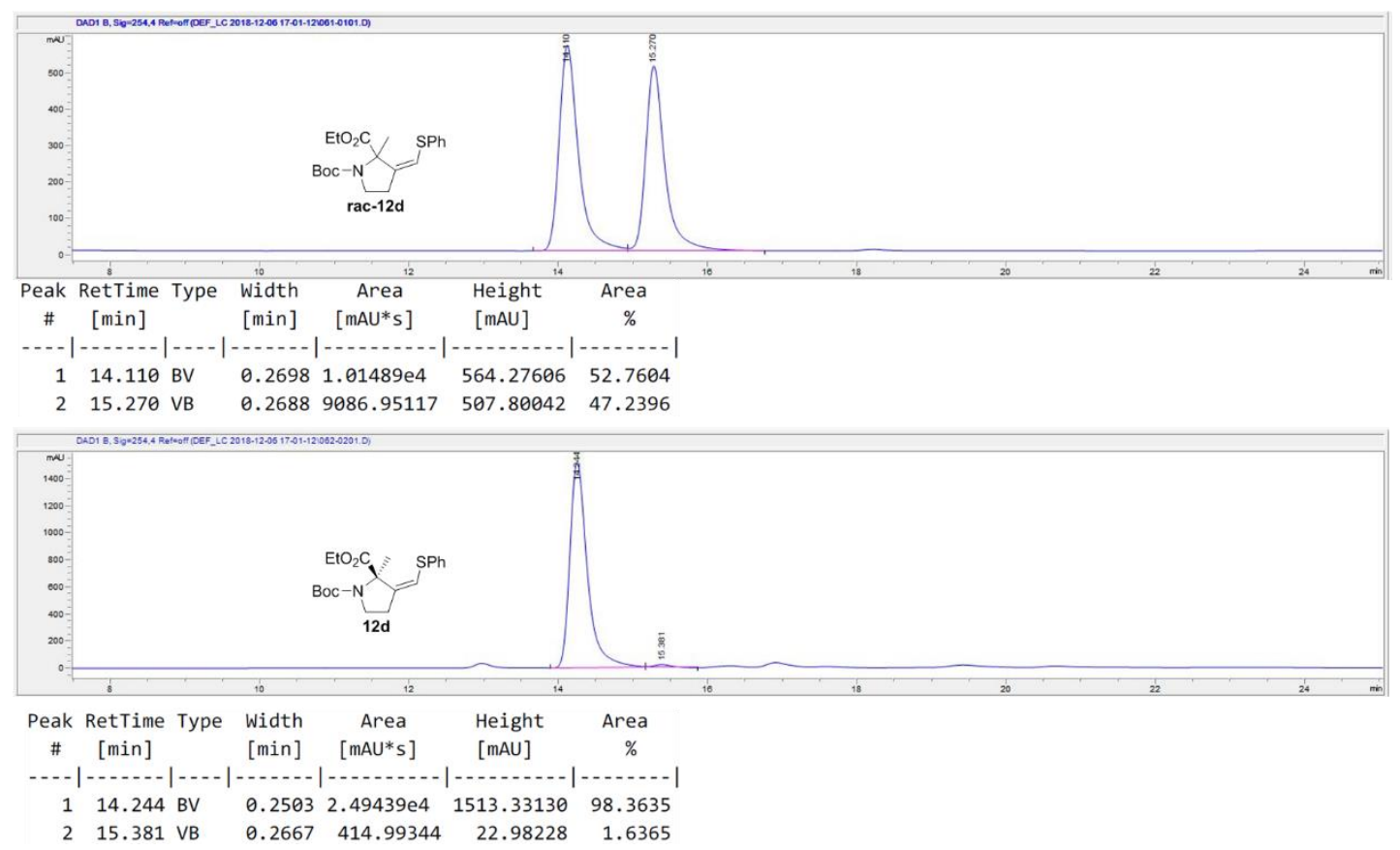


19. HPLC analysis: Chiralcel OD-H (hexane/ $/ \mathrm{PrOH}, 98 / 2,0.2 \mathrm{~mL} / \mathrm{min}, 254 \mathrm{~nm}, 25^{\circ} \mathrm{C}$ ); 30.1 (minor), 31.1 (major) $\min ; 98 \%$ ee.

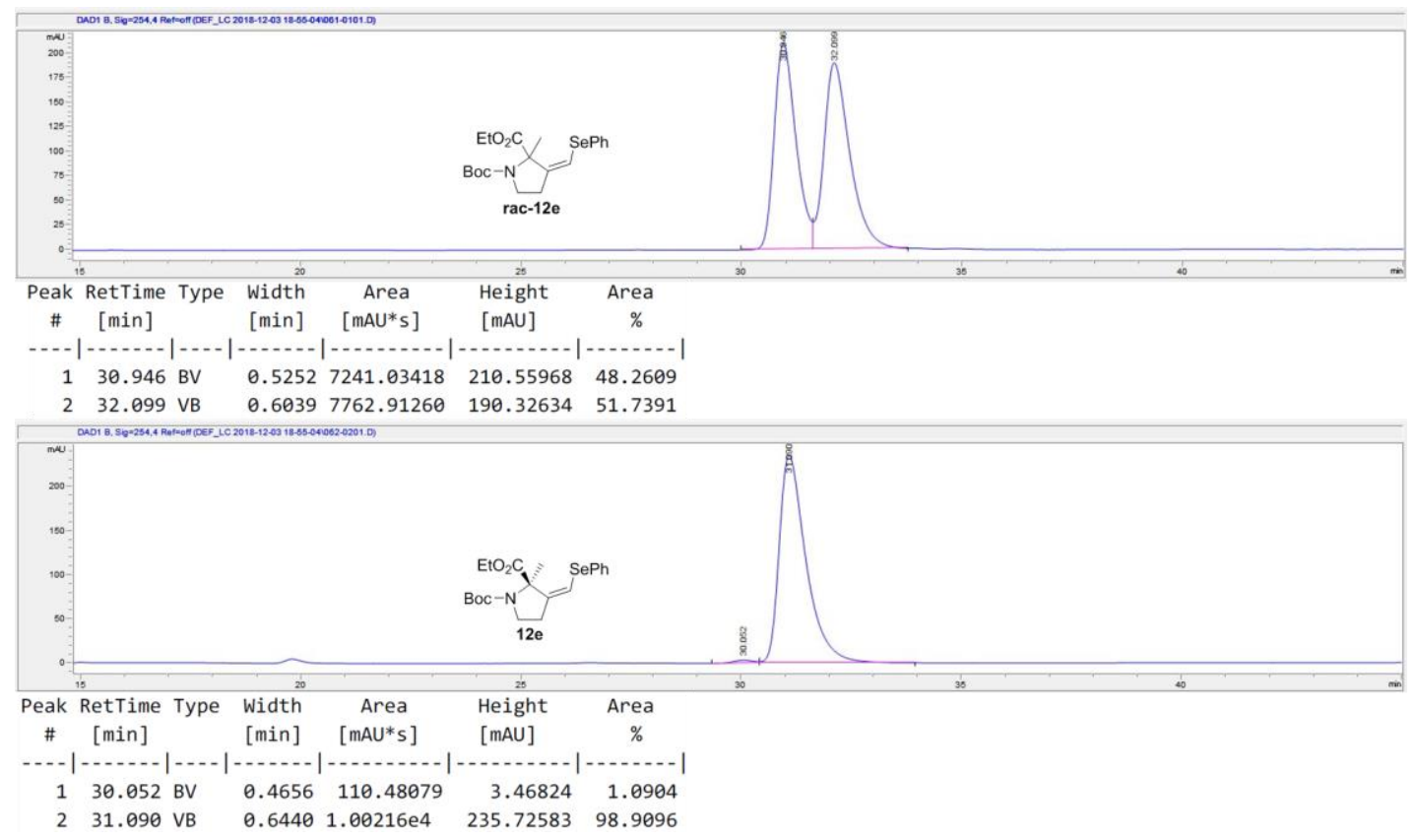

20. HPLC analysis: Because 12f can not be fully separated after trying a few chiral columns and conditions, we transform 12f to 12f' for HPLC analysis. Chiralcel OD-H (hexane/ ${ }^{i} \mathrm{PrOH}, 80 / 20,0.5 \mathrm{~mL} / \mathrm{min}, 254 \mathrm{~nm}, 25^{\circ} \mathrm{C}$ ); 9.6 (minor), 10.1 (major) min; 94\% ee.

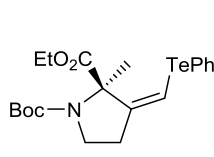

$12 \mathrm{f}$

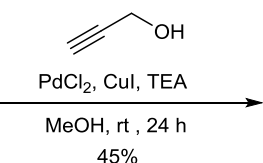

$45 \%$
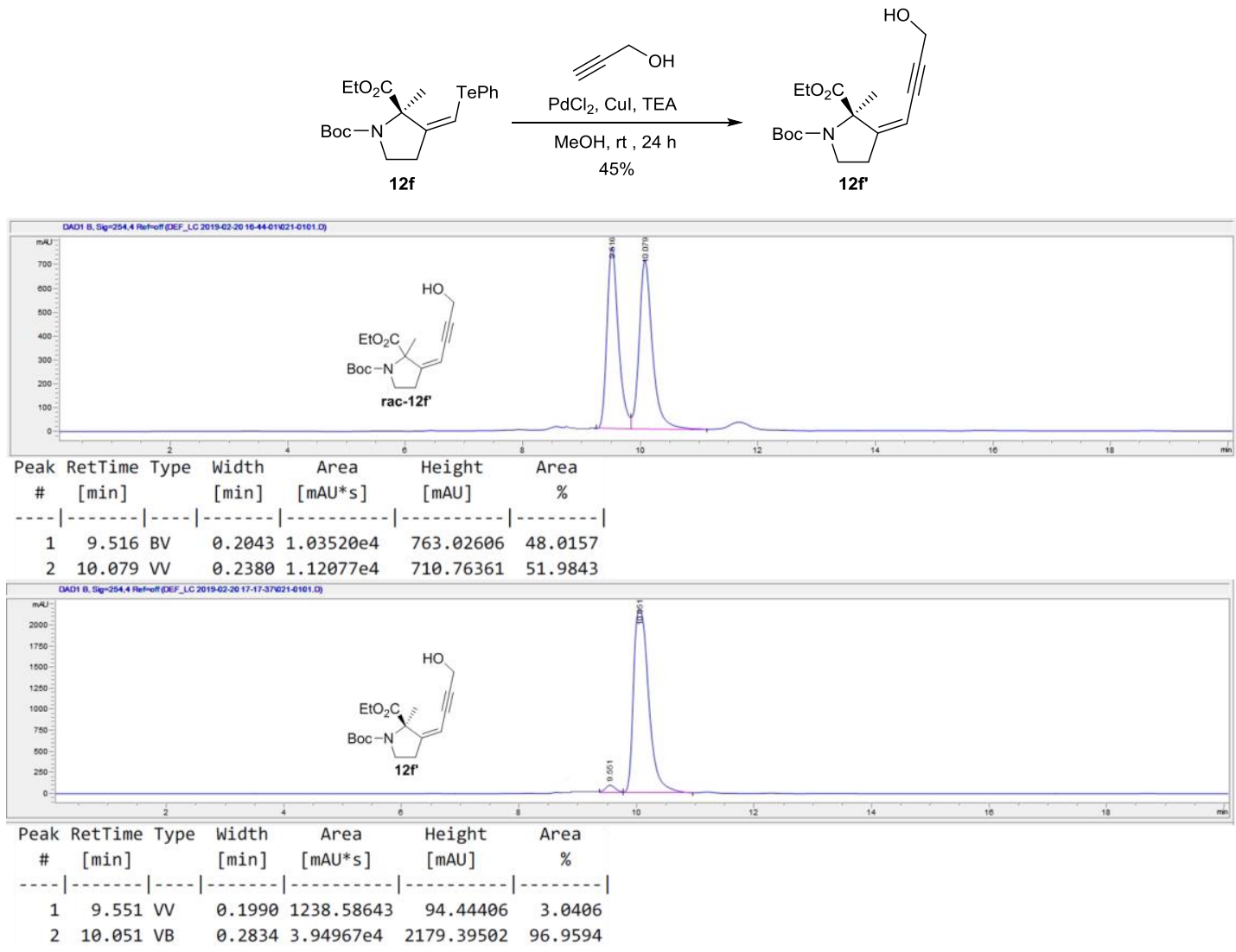


\section{4. ${ }^{1} \mathrm{H}$ NMR and ${ }^{13} \mathrm{C}$ NMR Spectra}
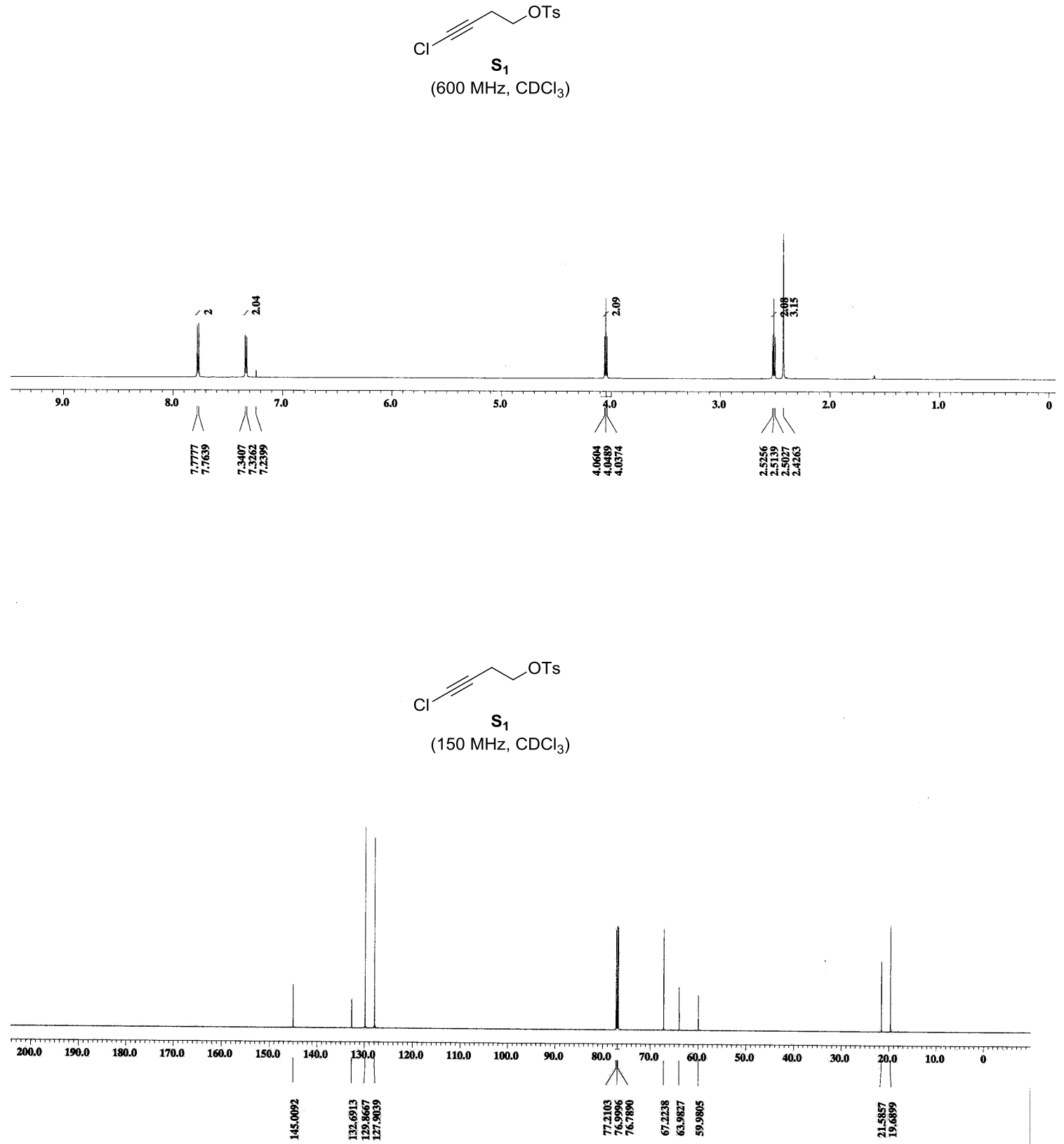


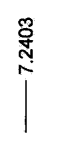
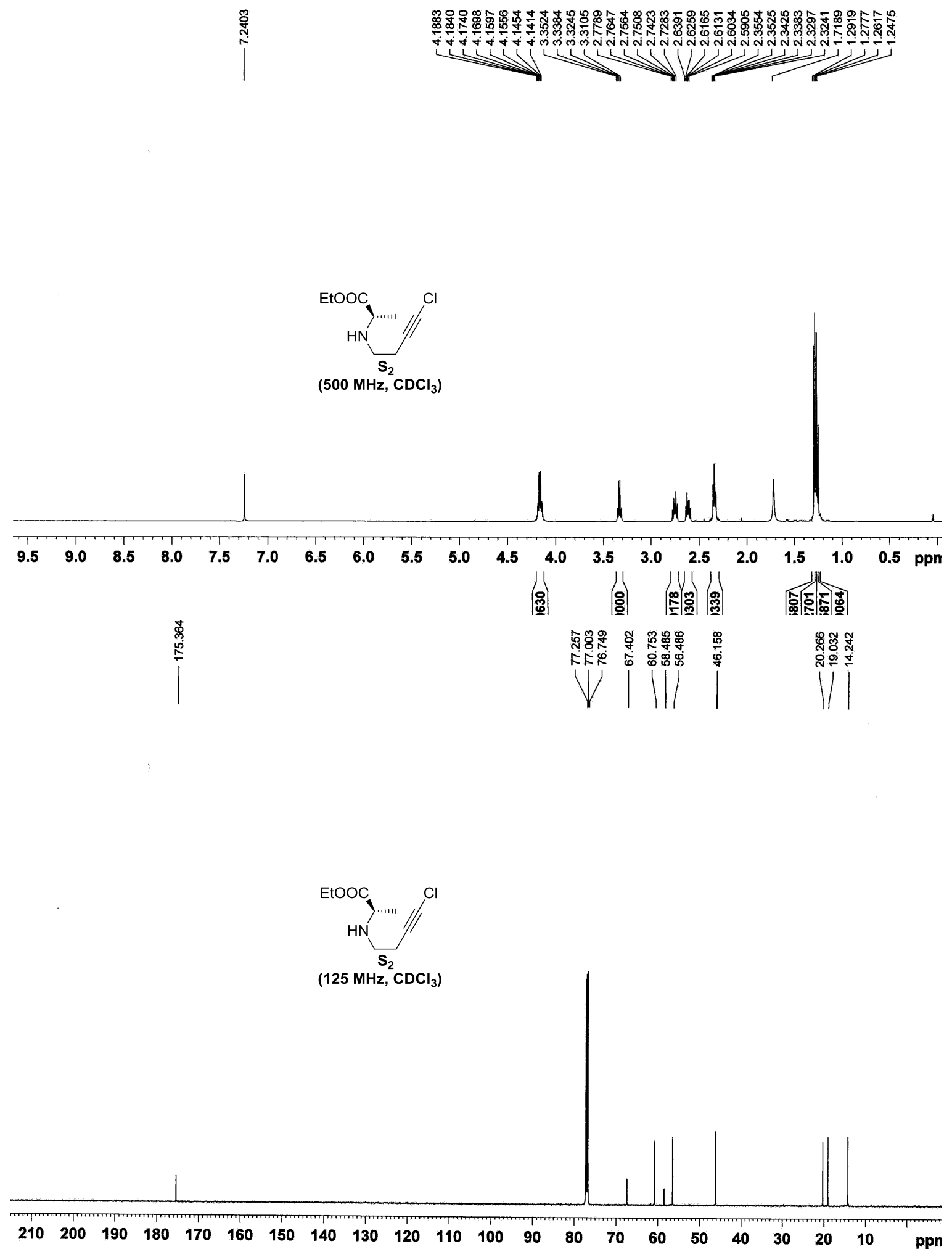

S22 


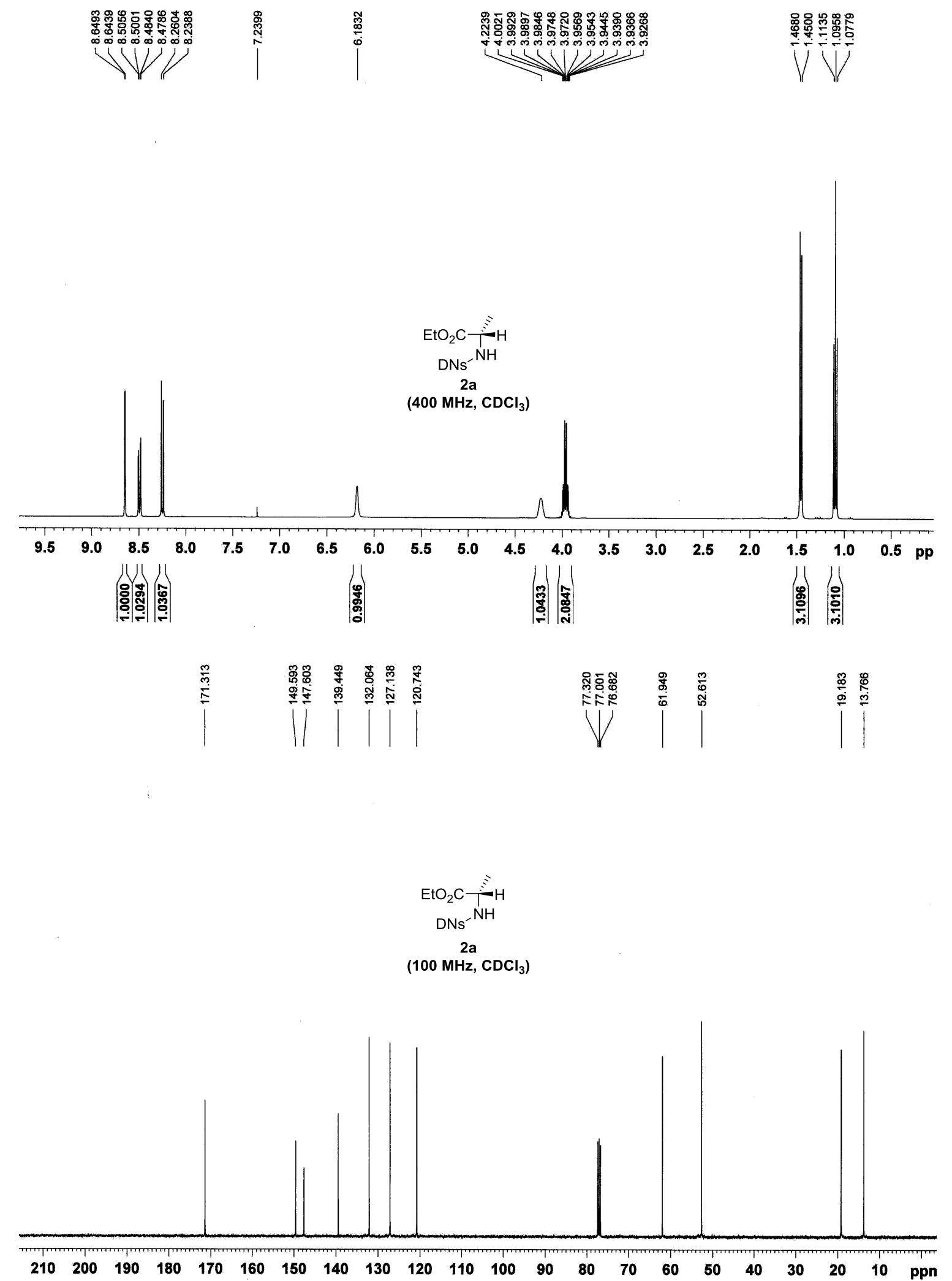




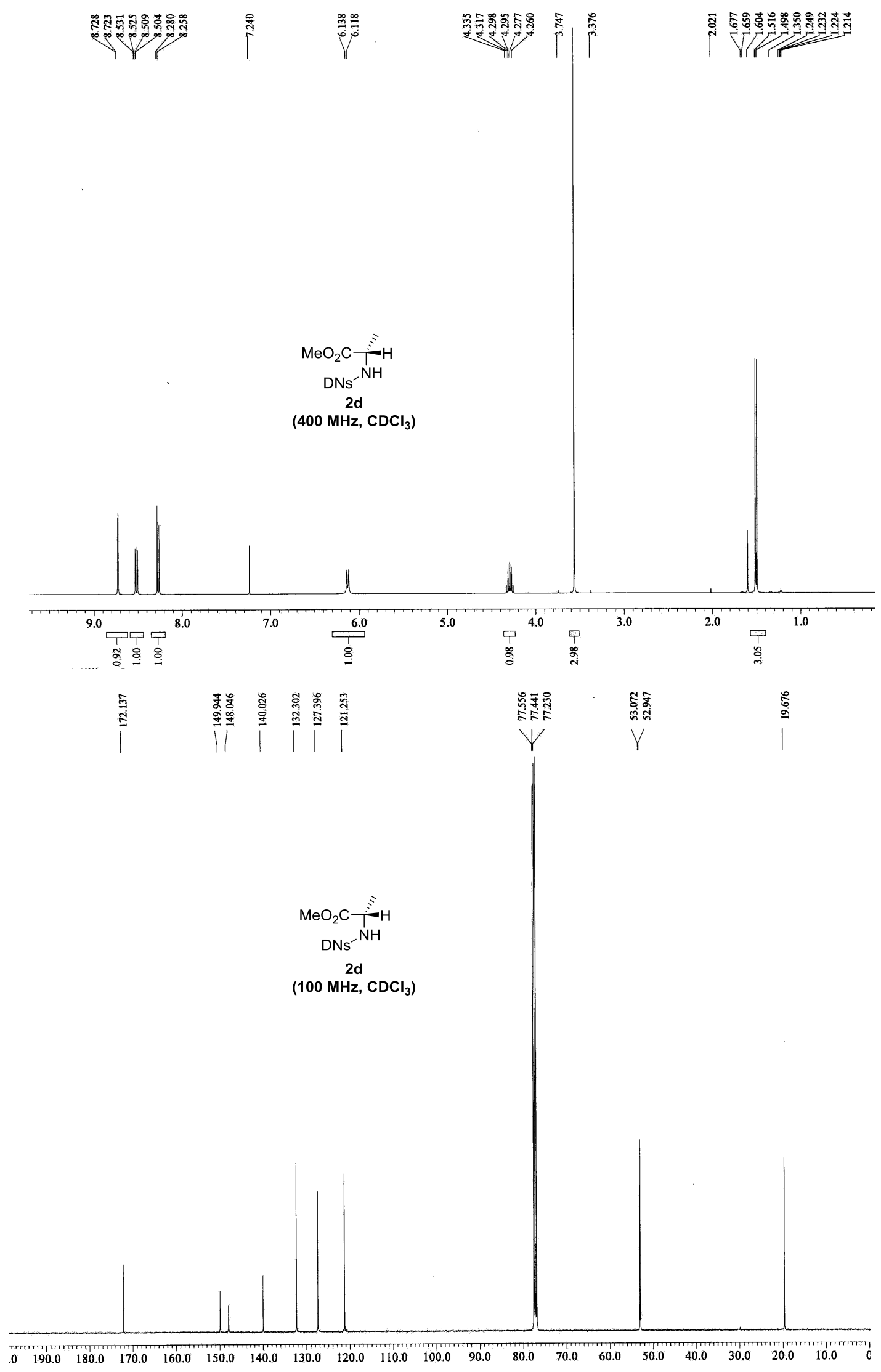



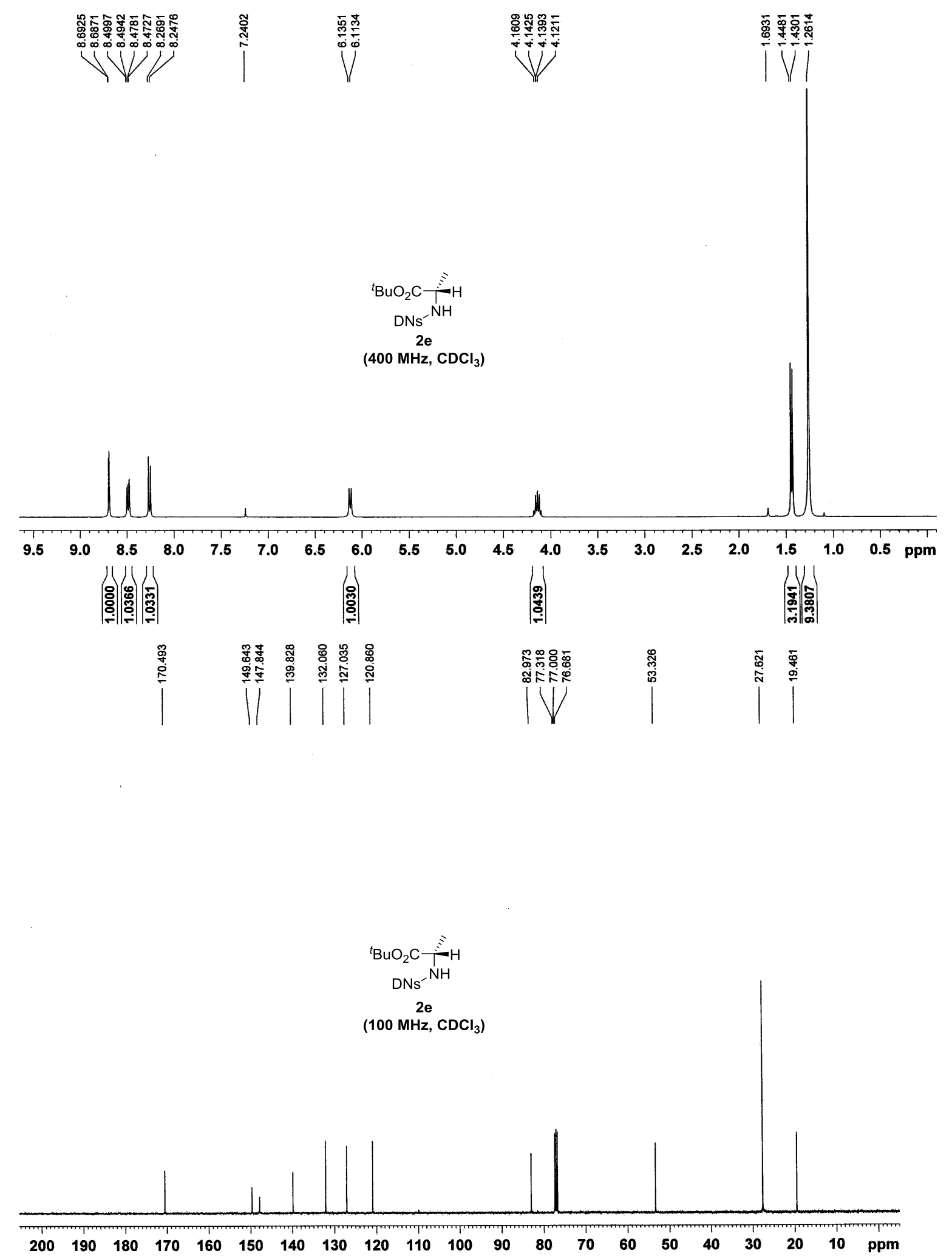


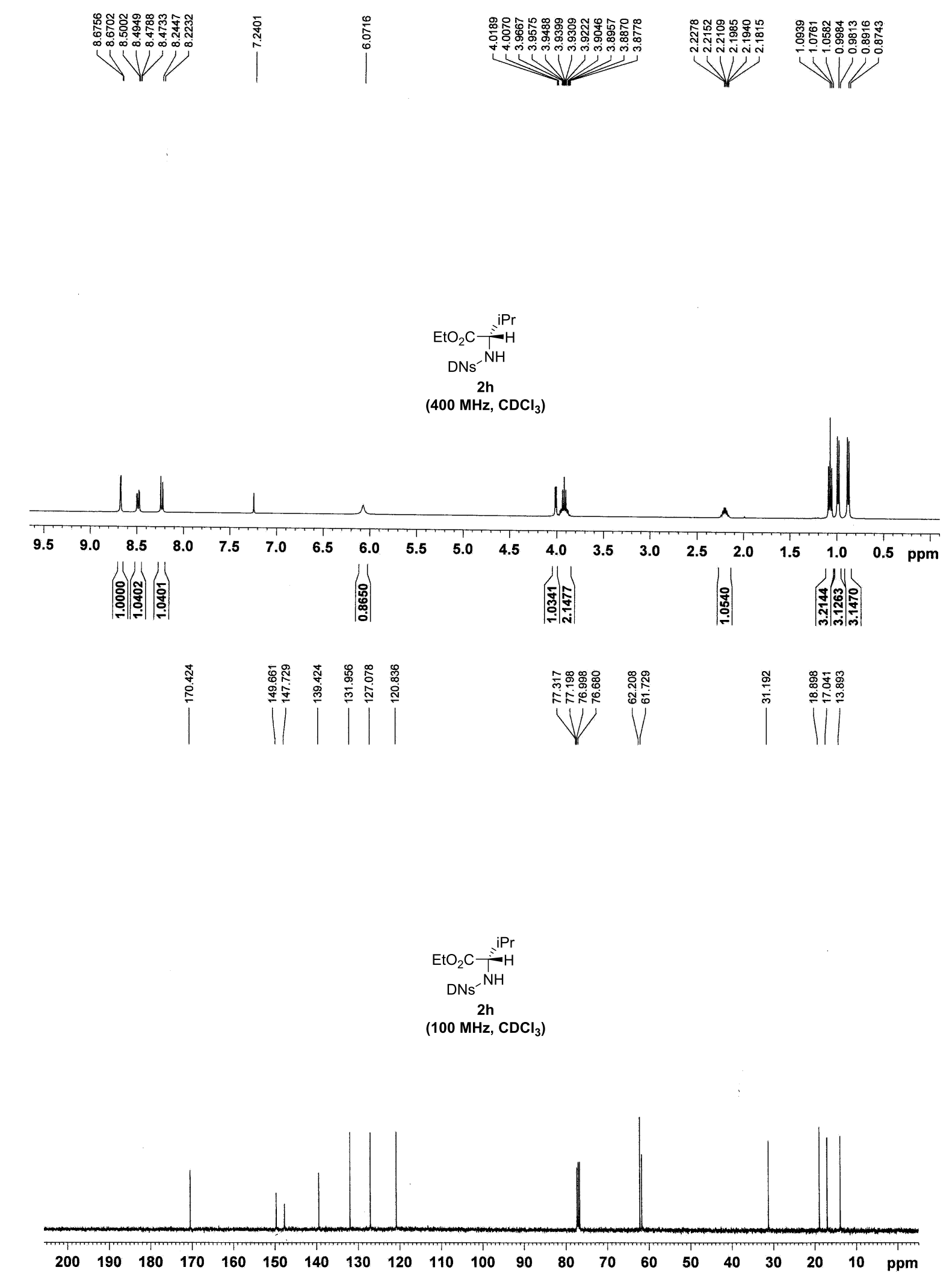




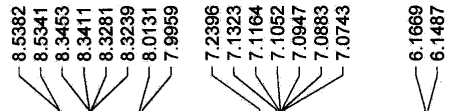
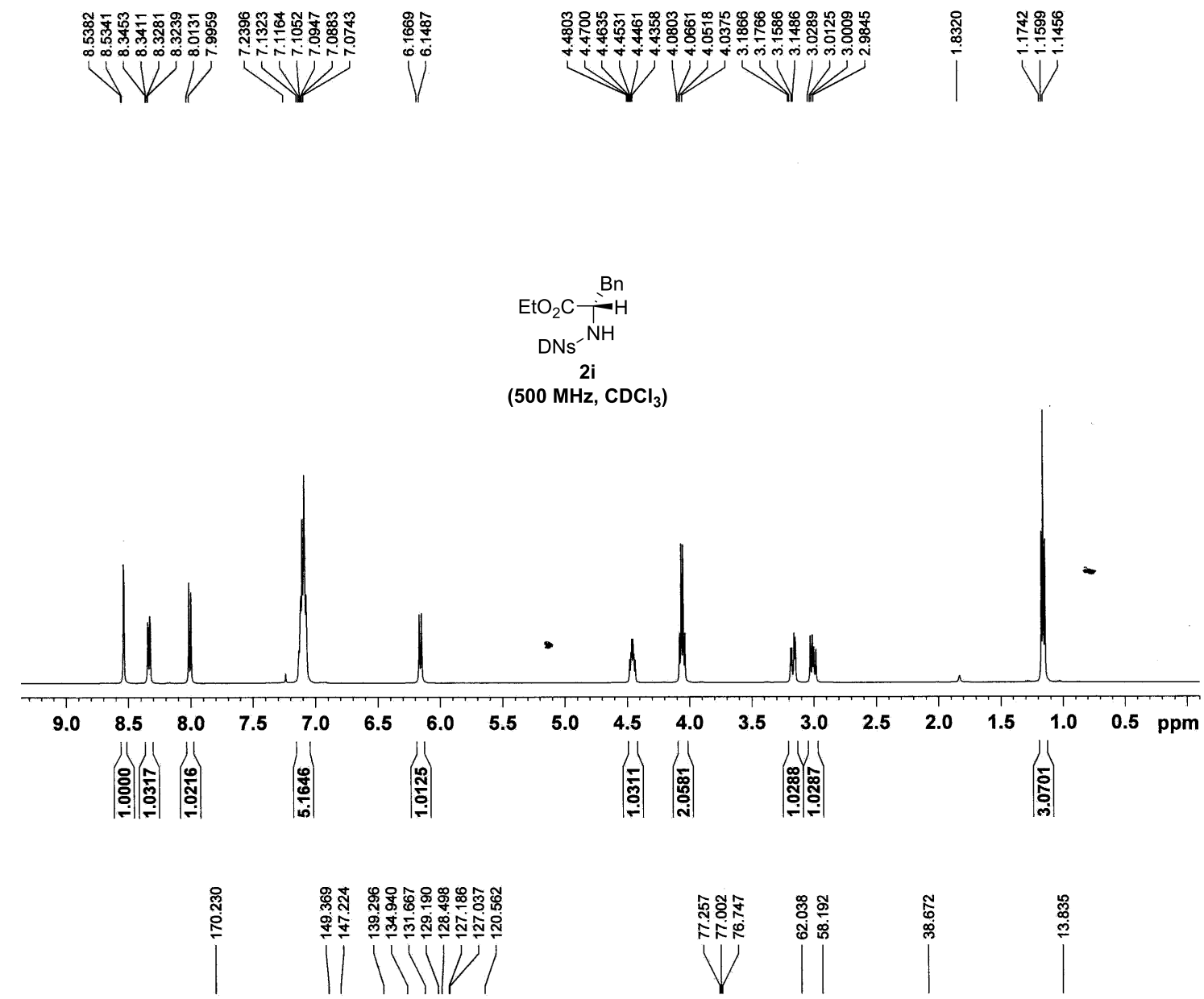

$$
\mathrm{DNs}_{2}-\overbrace{-}^{\mathrm{NH}} \mathrm{HH}
$$

(125 MHz, $\mathrm{CDCl}_{3}$ )

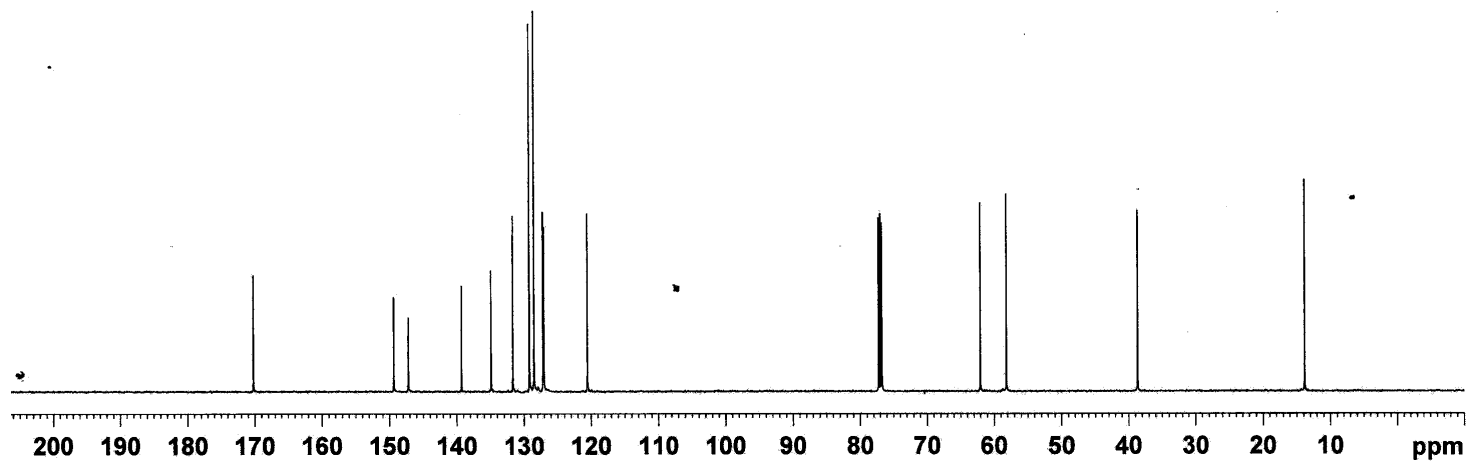



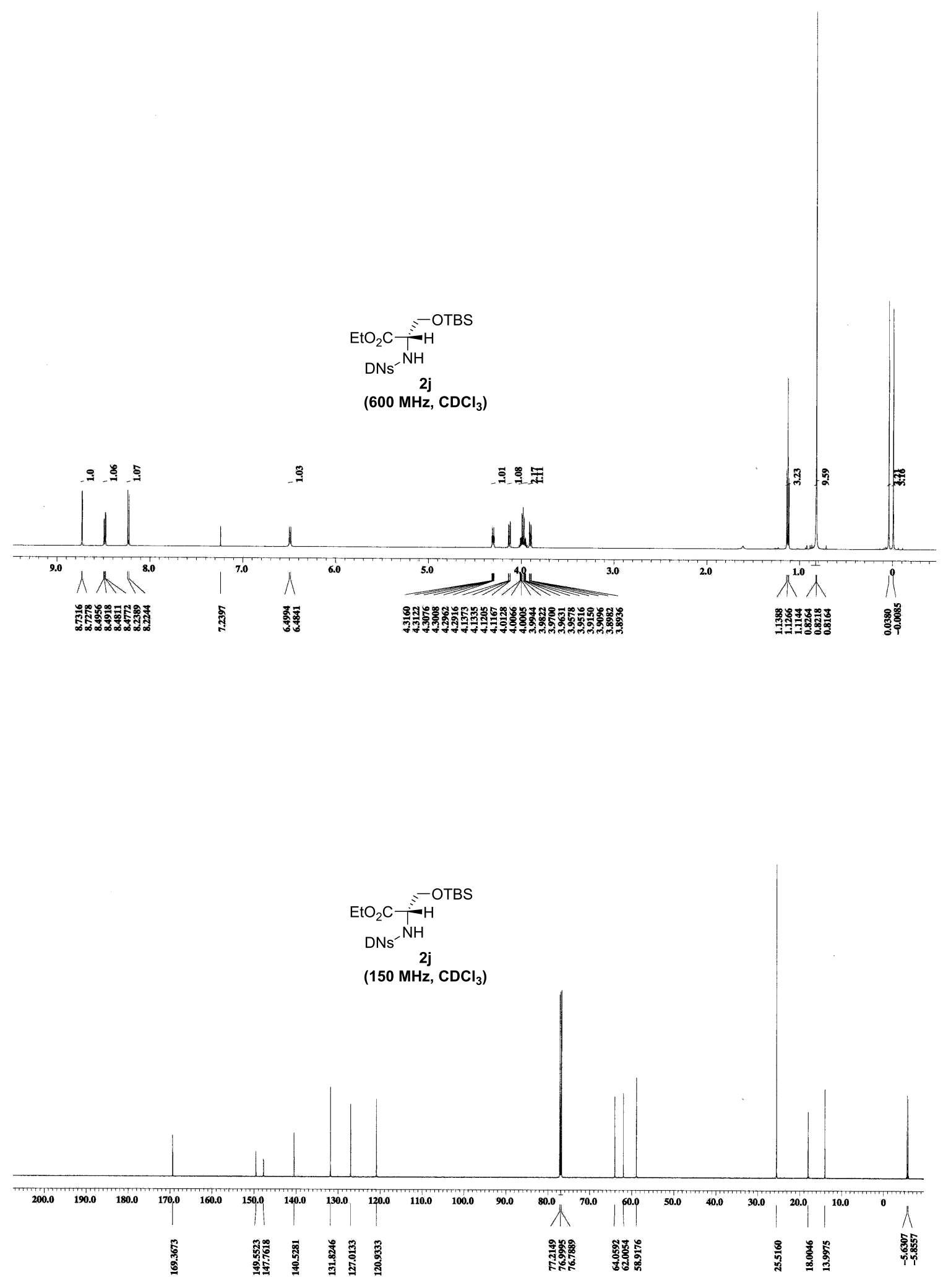


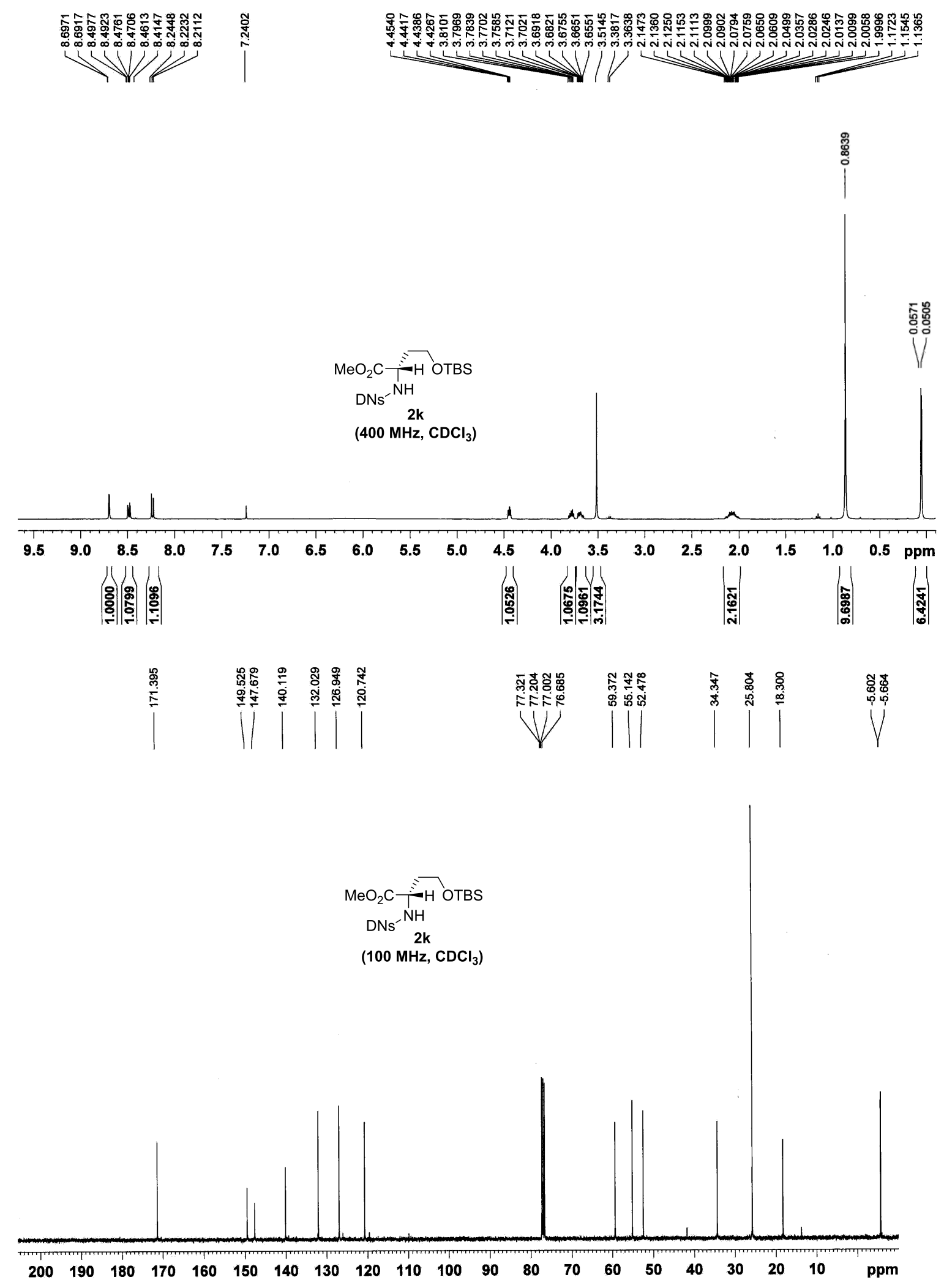




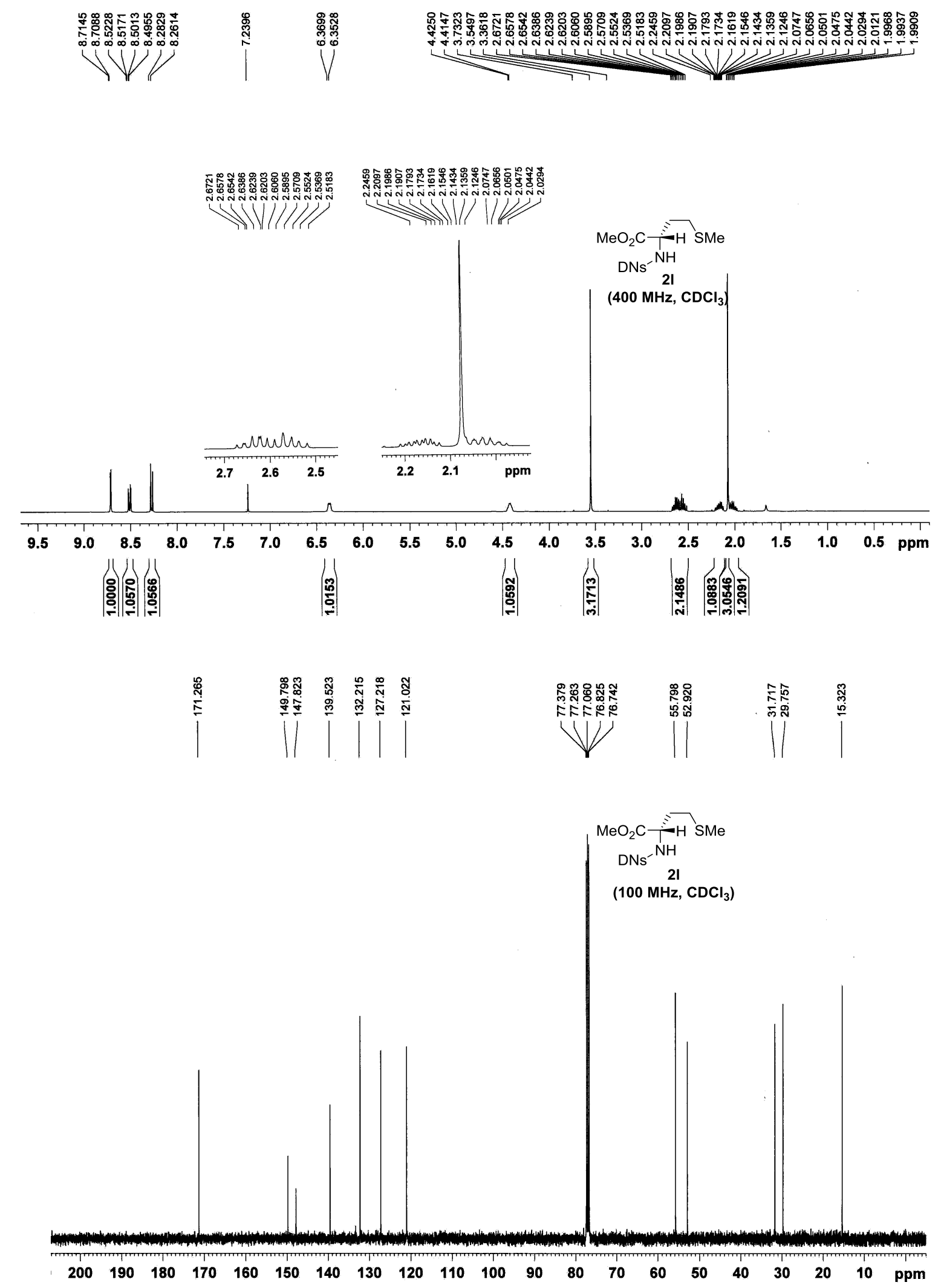



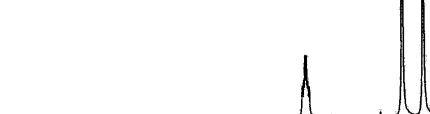

\begin{tabular}{llll|}
$\mathbf{9 . 0}$ & $\mathbf{8 . 5}$ & $\mathbf{8 . 0}$ & $\mathbf{7 . 5}$
\end{tabular}

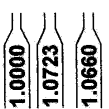
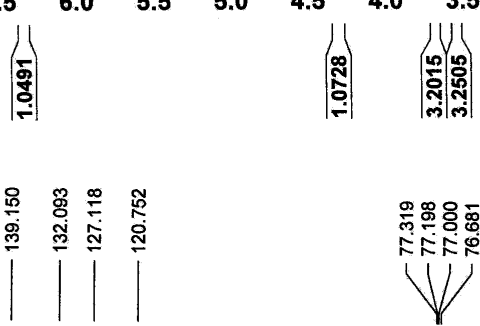

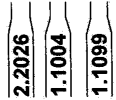
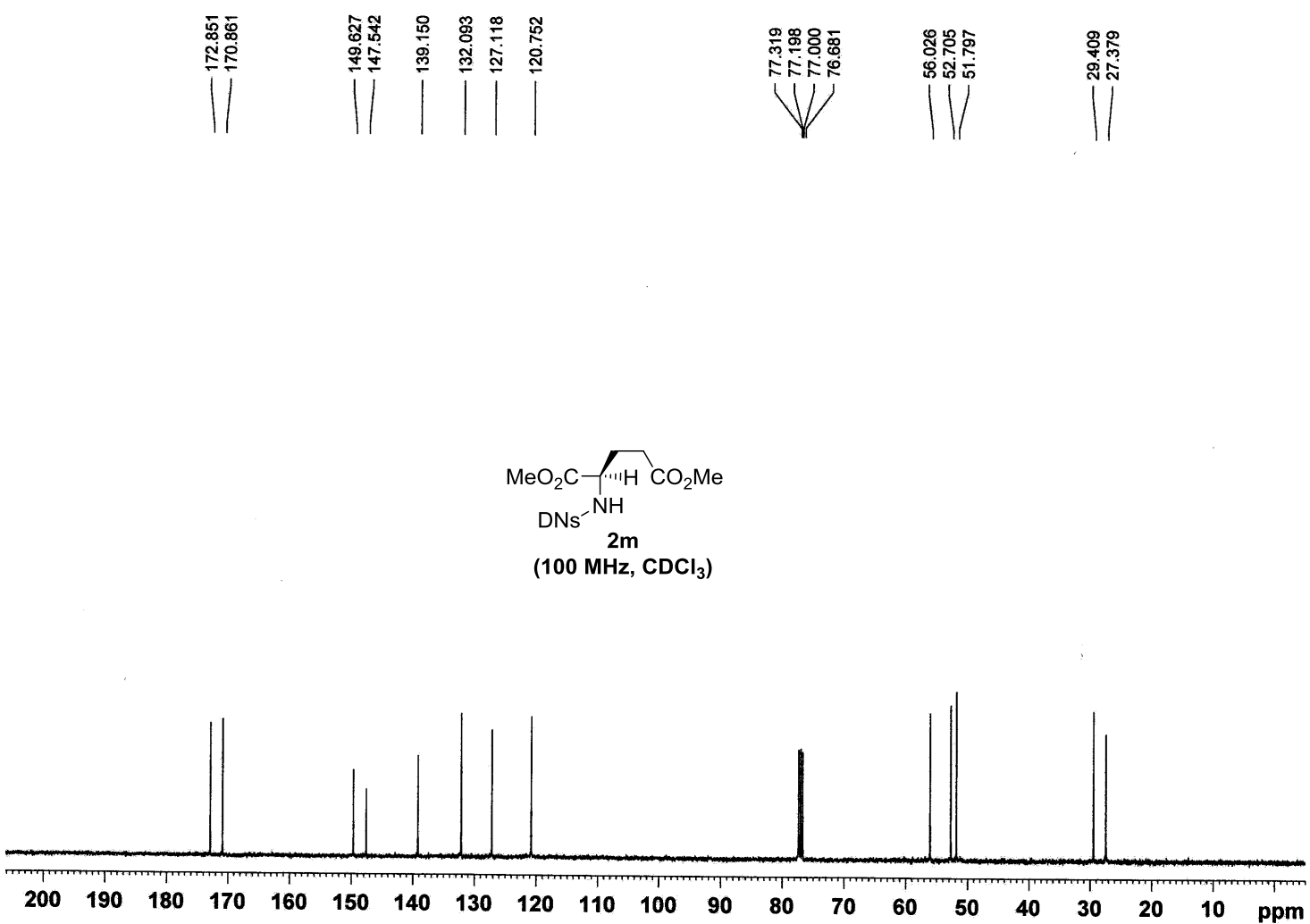

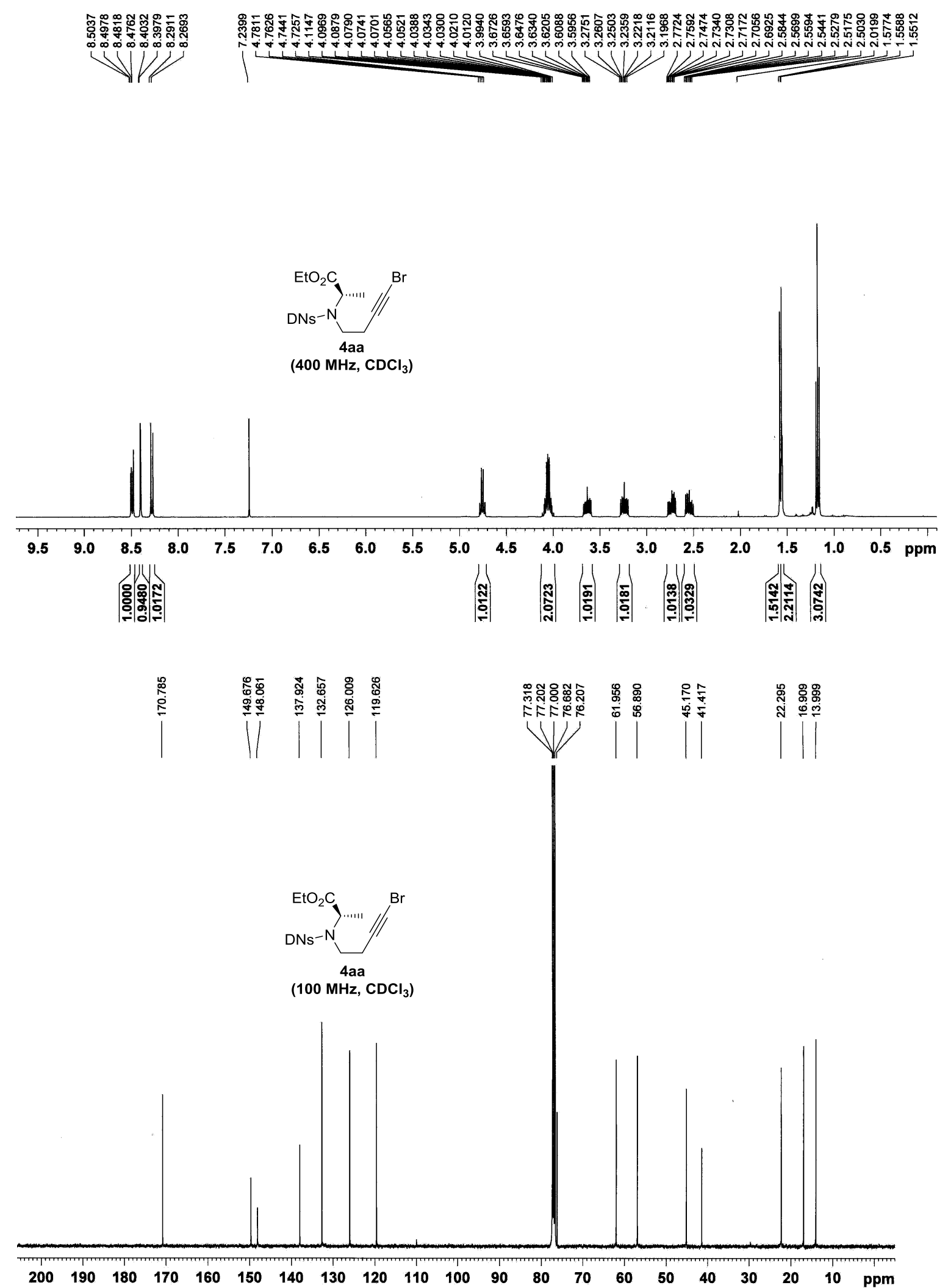


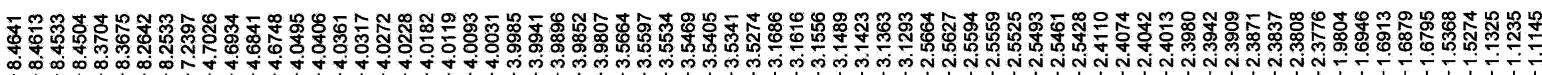
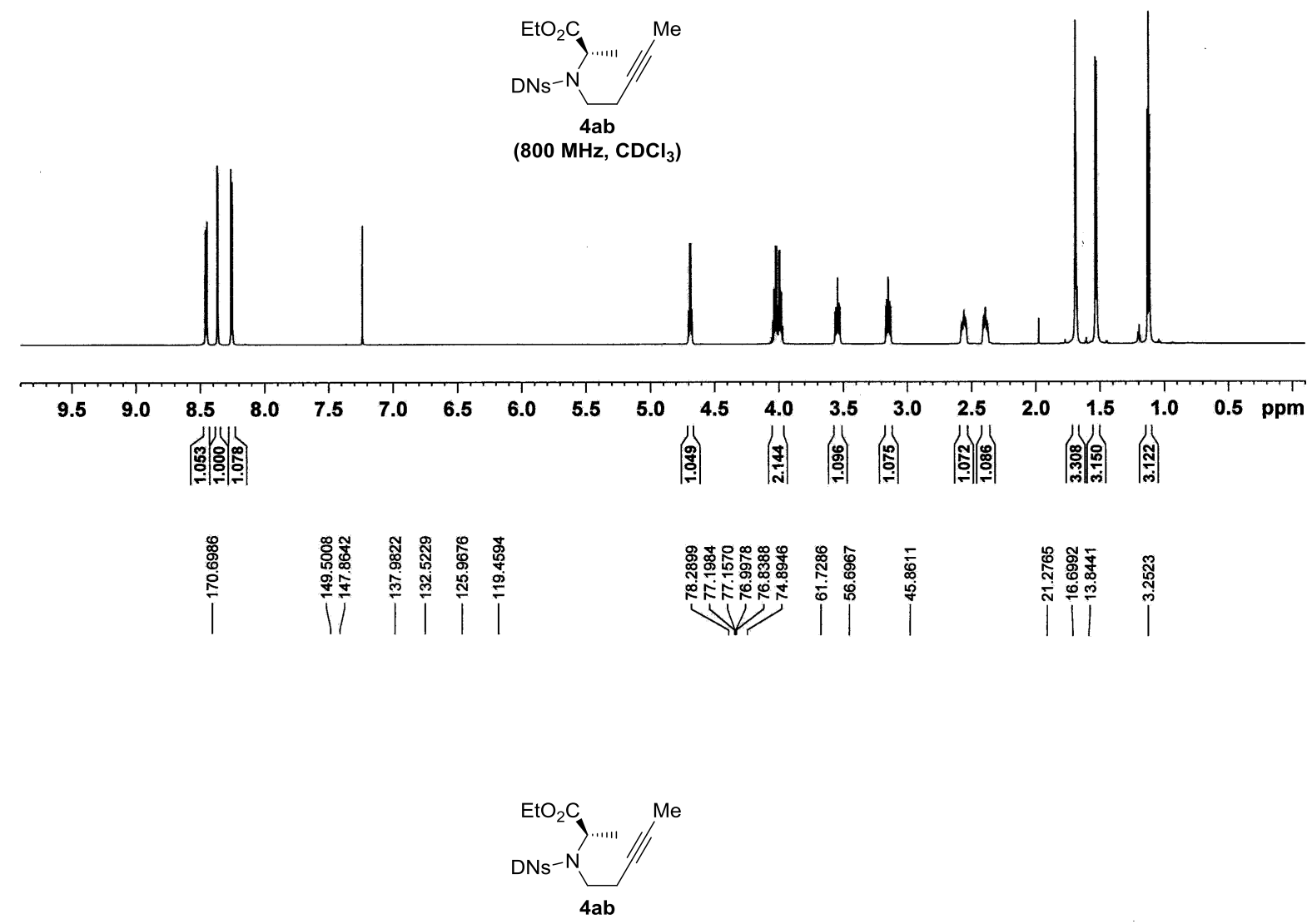

(200 MHz, $\mathrm{CDCl}_{3}$ )

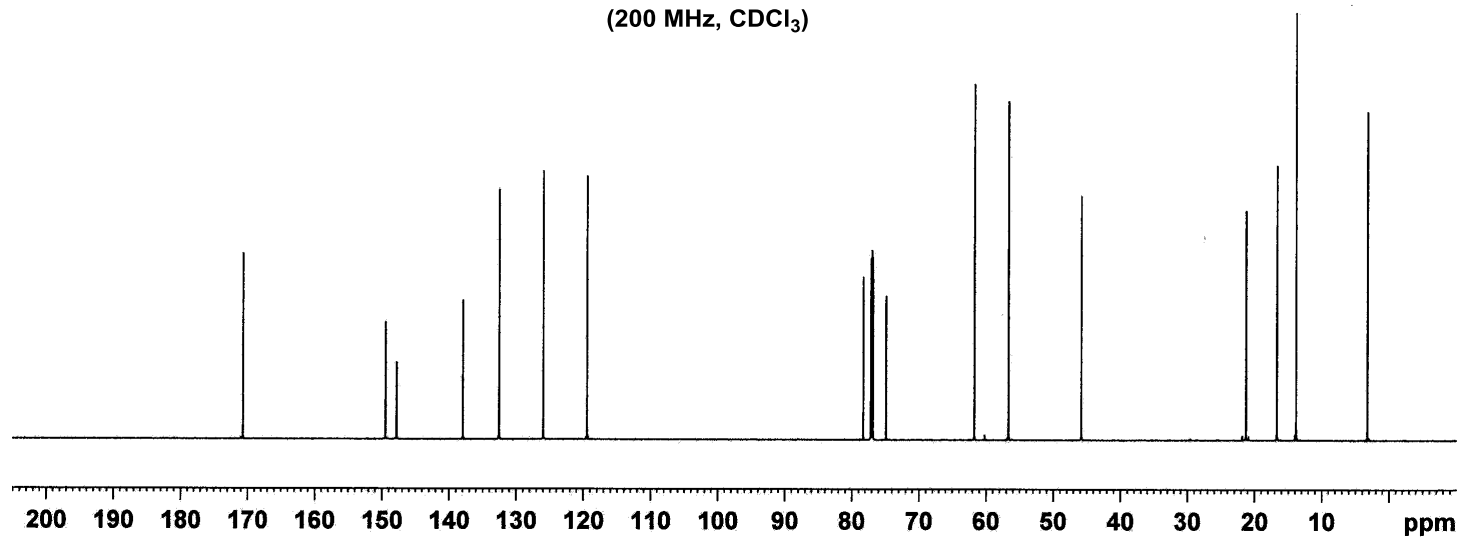




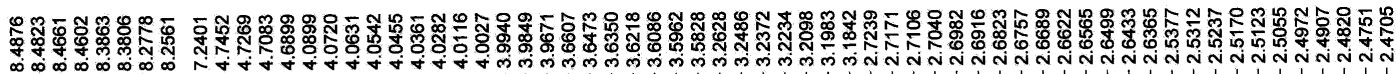
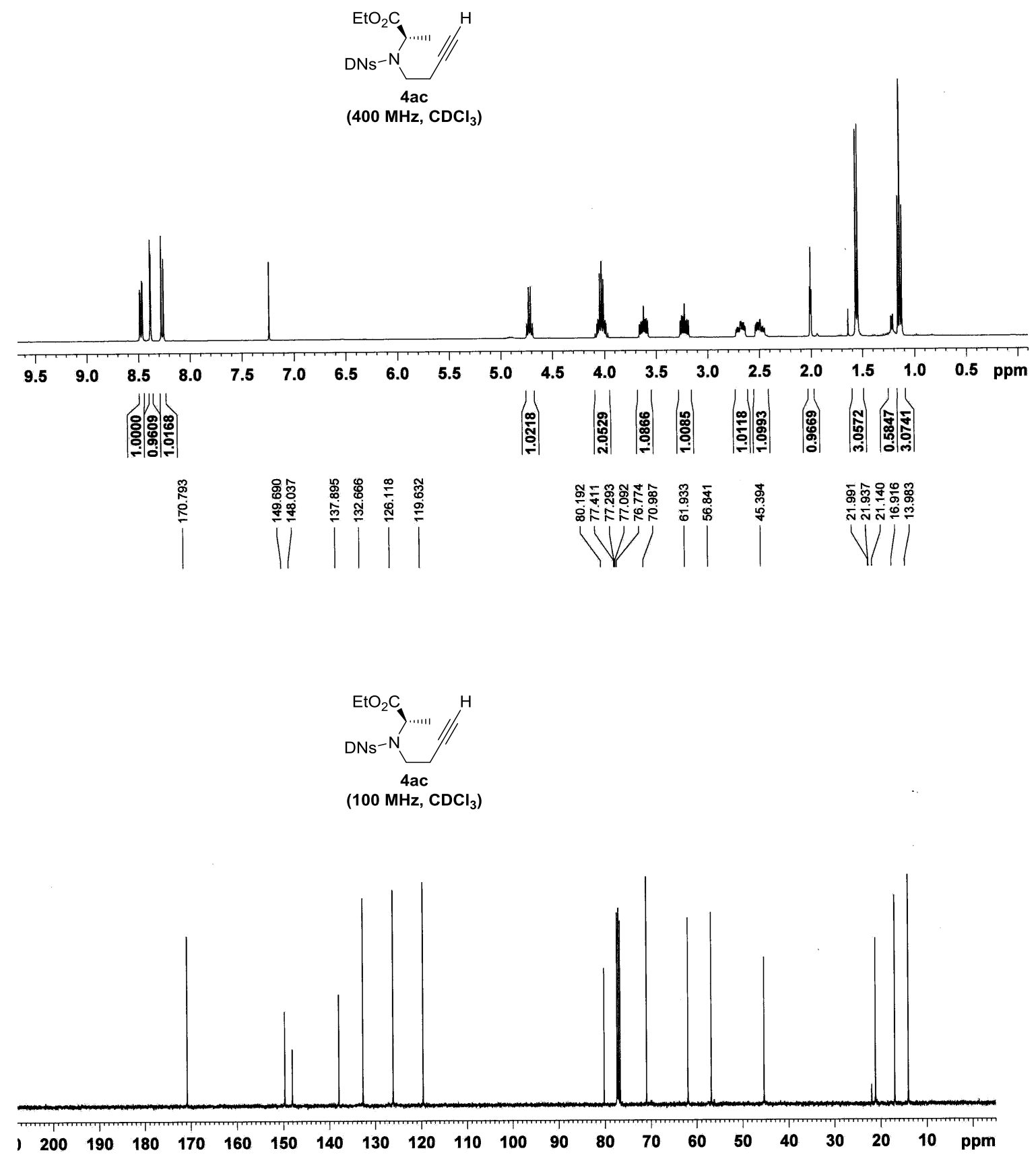


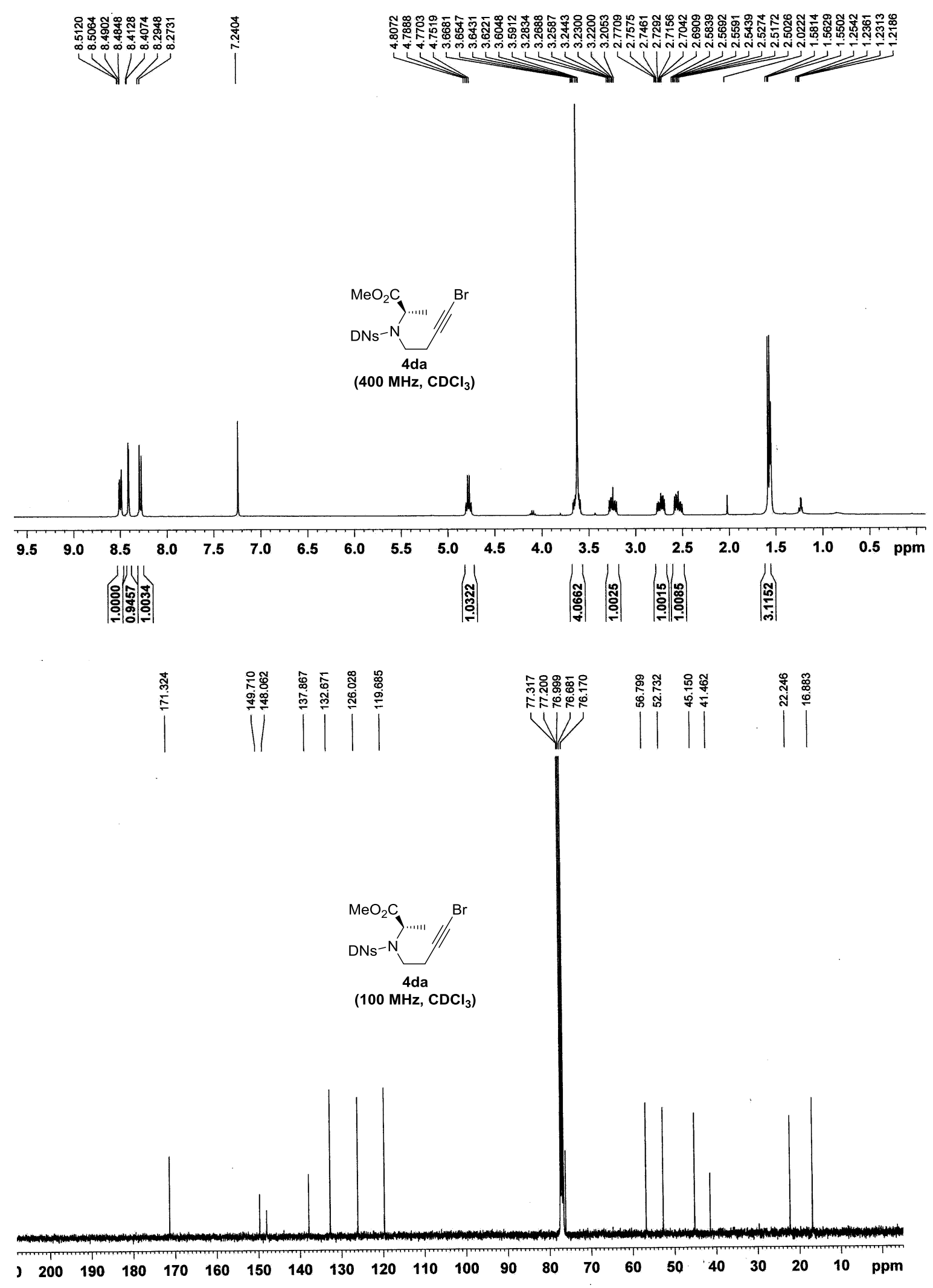




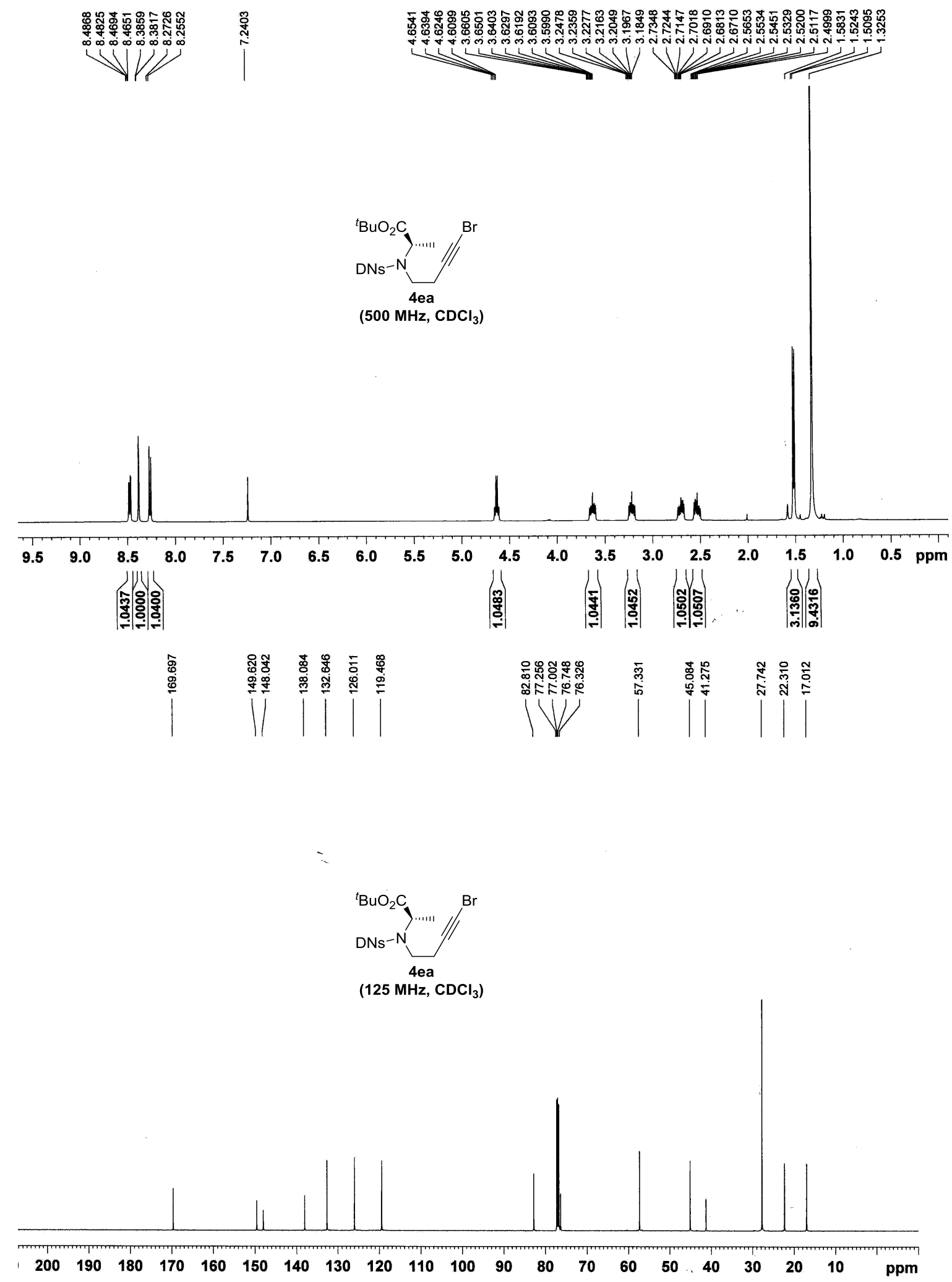




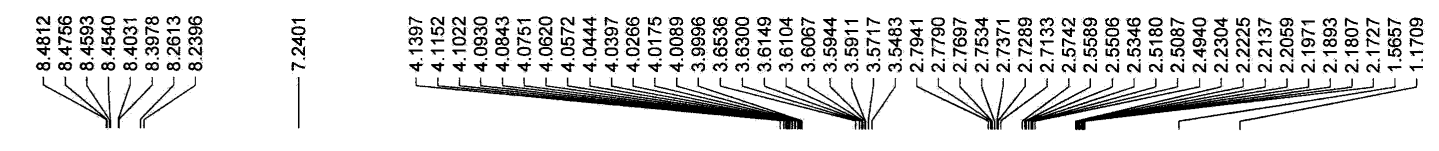

$\left(\mathbf{4 0 0} \mathbf{~} \mathbf{4 h z}, \mathrm{CDCl}_{3}\right)$
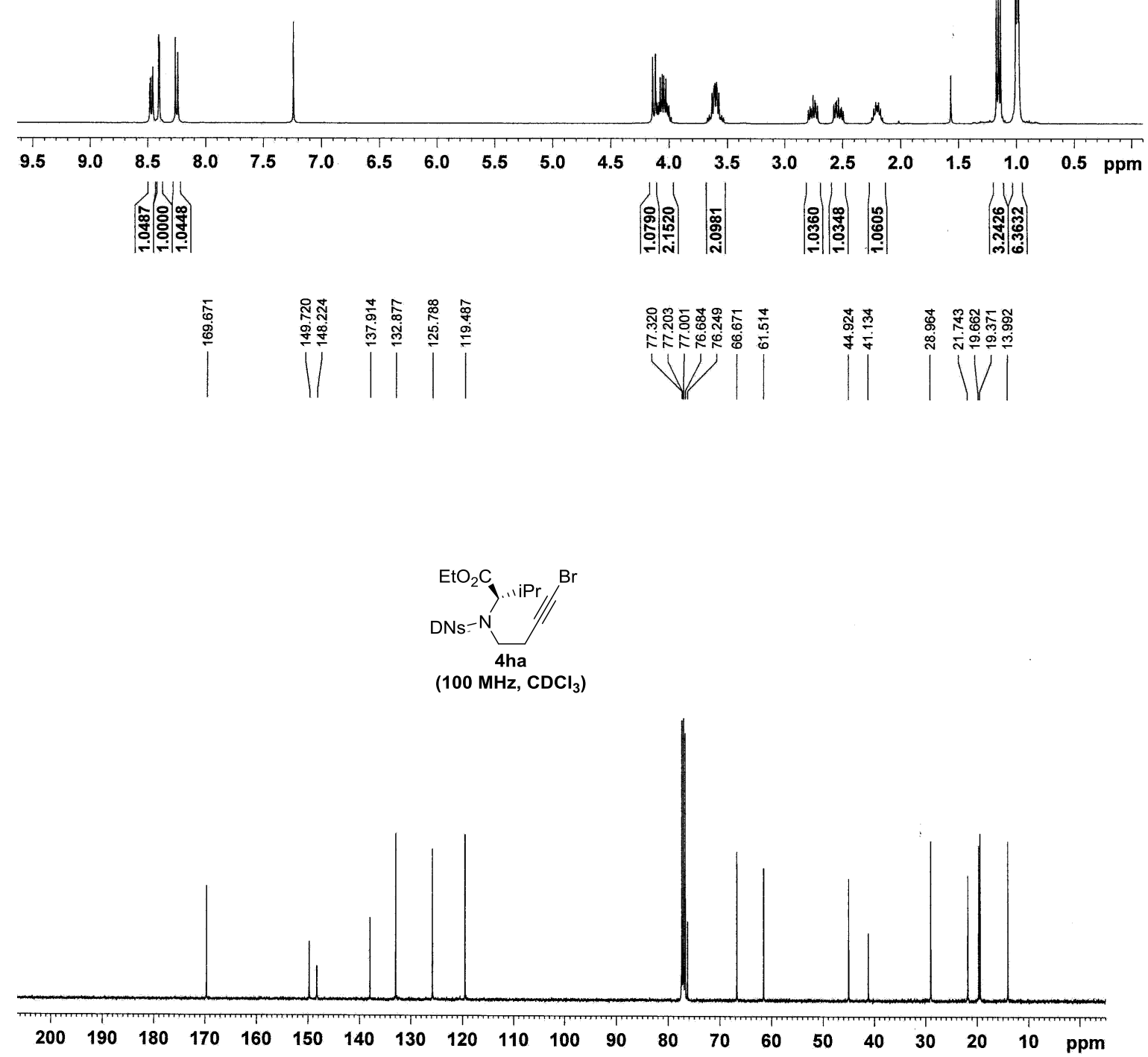


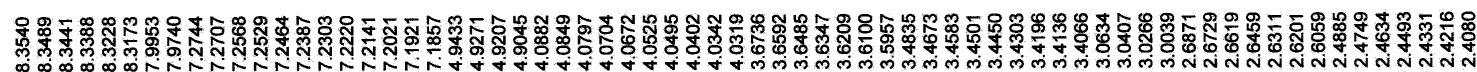
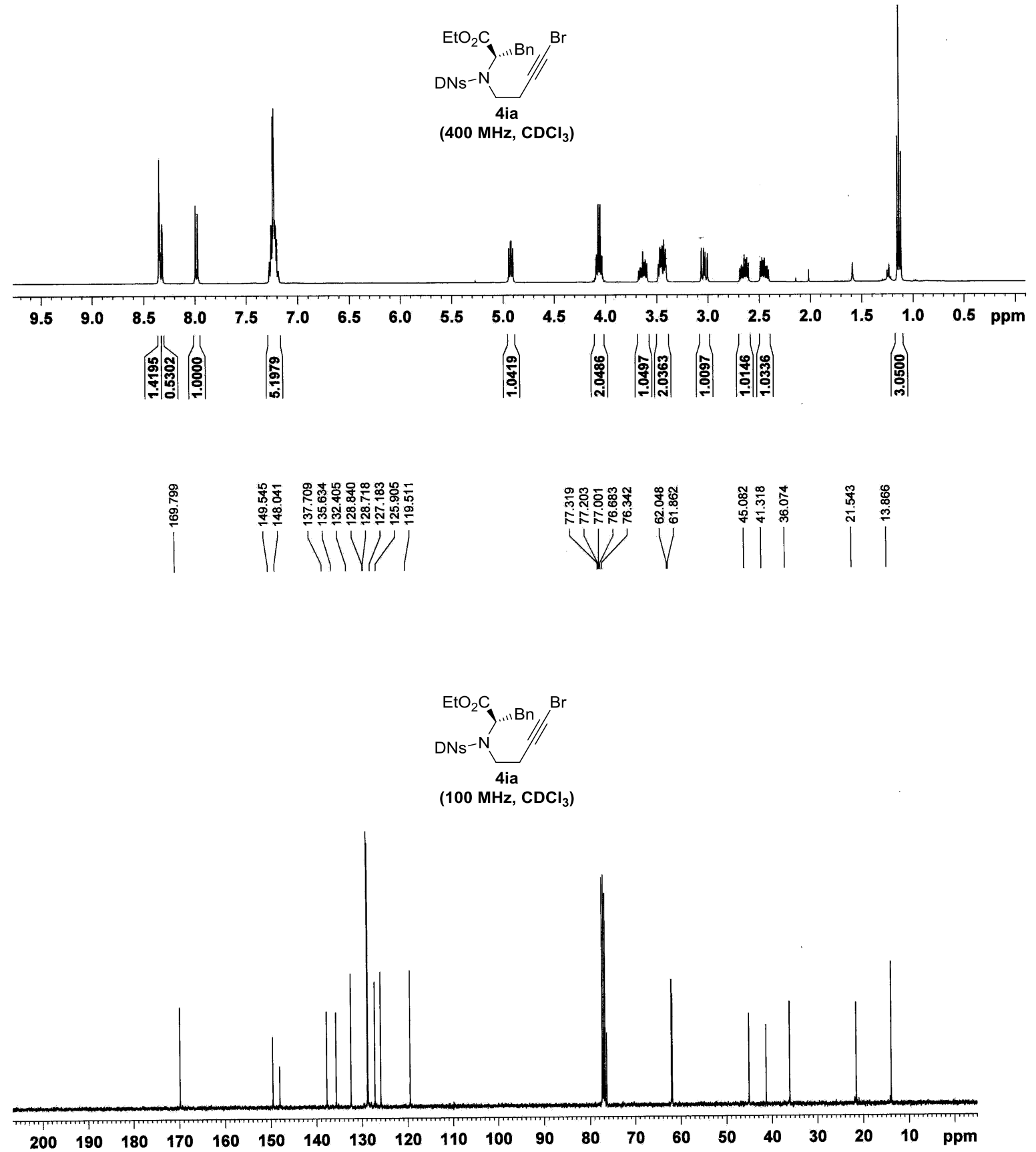


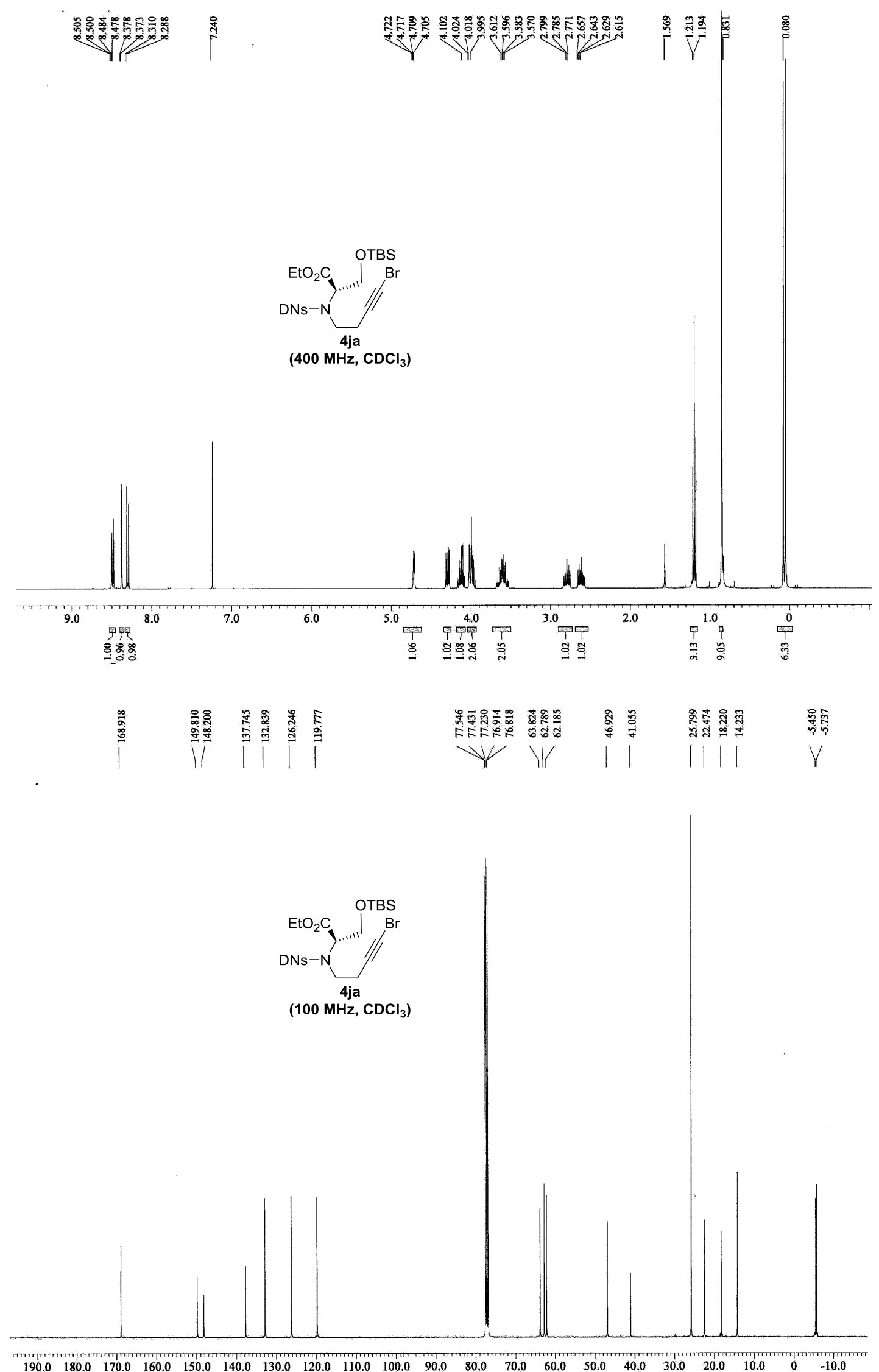




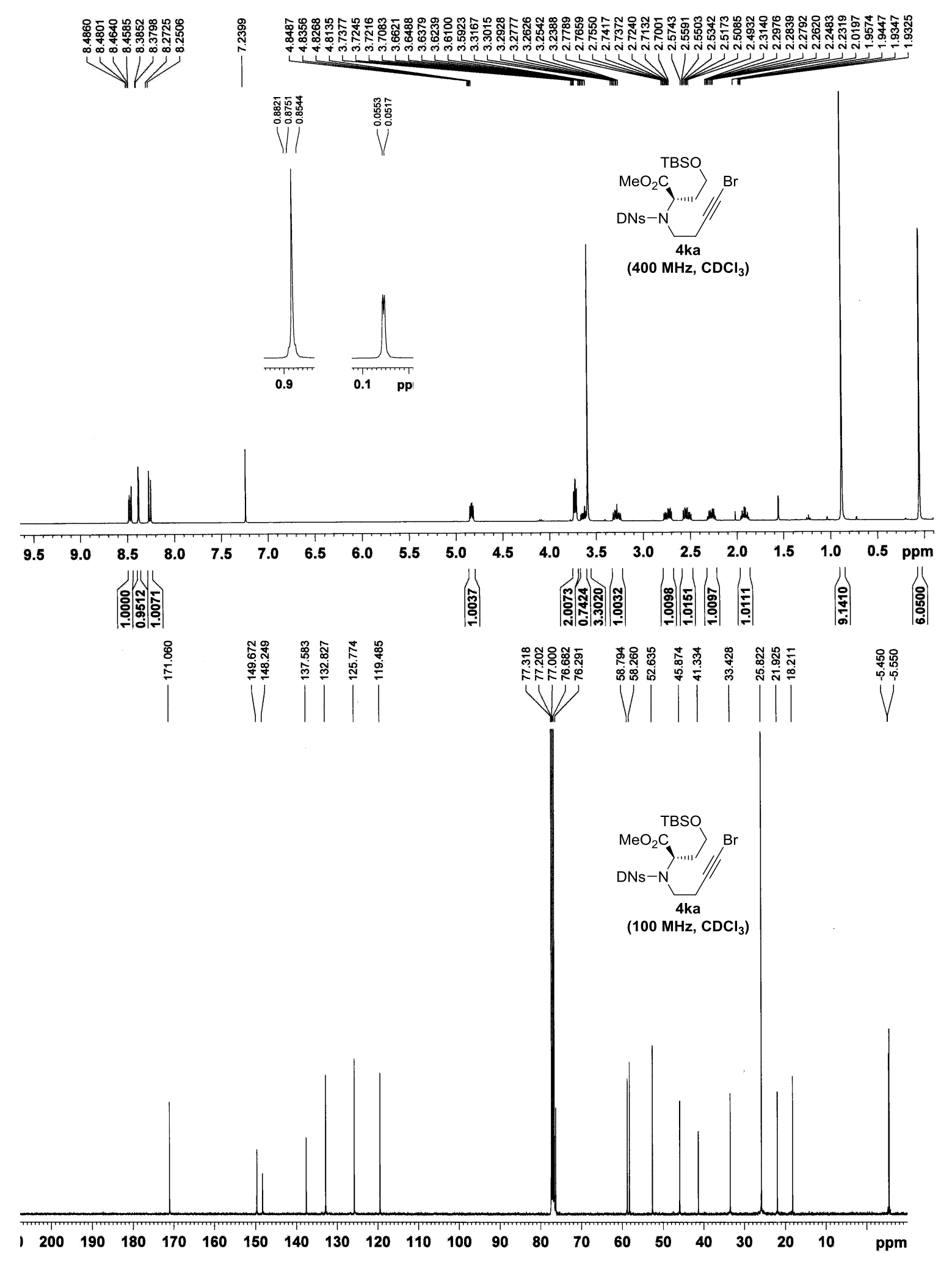




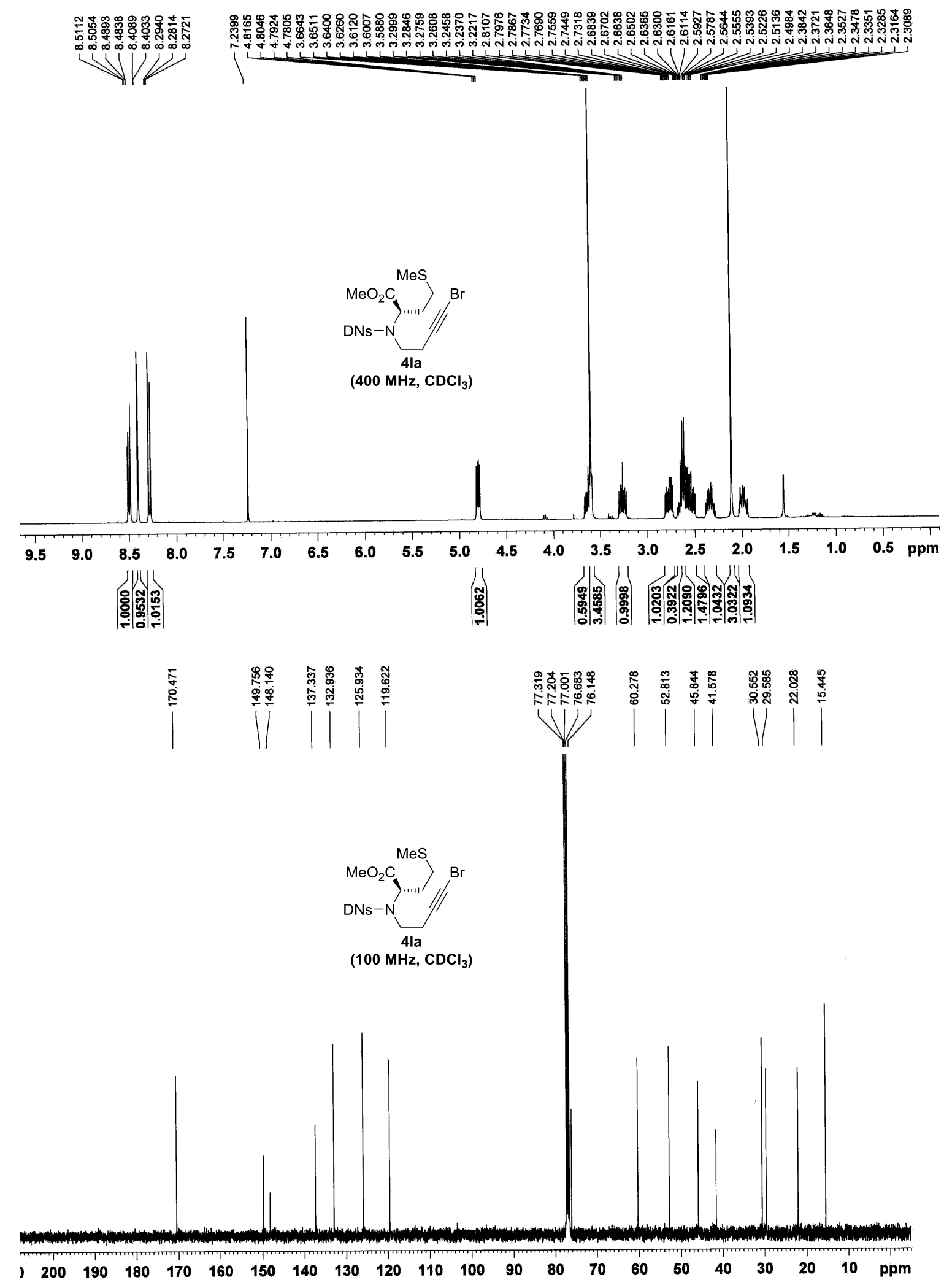




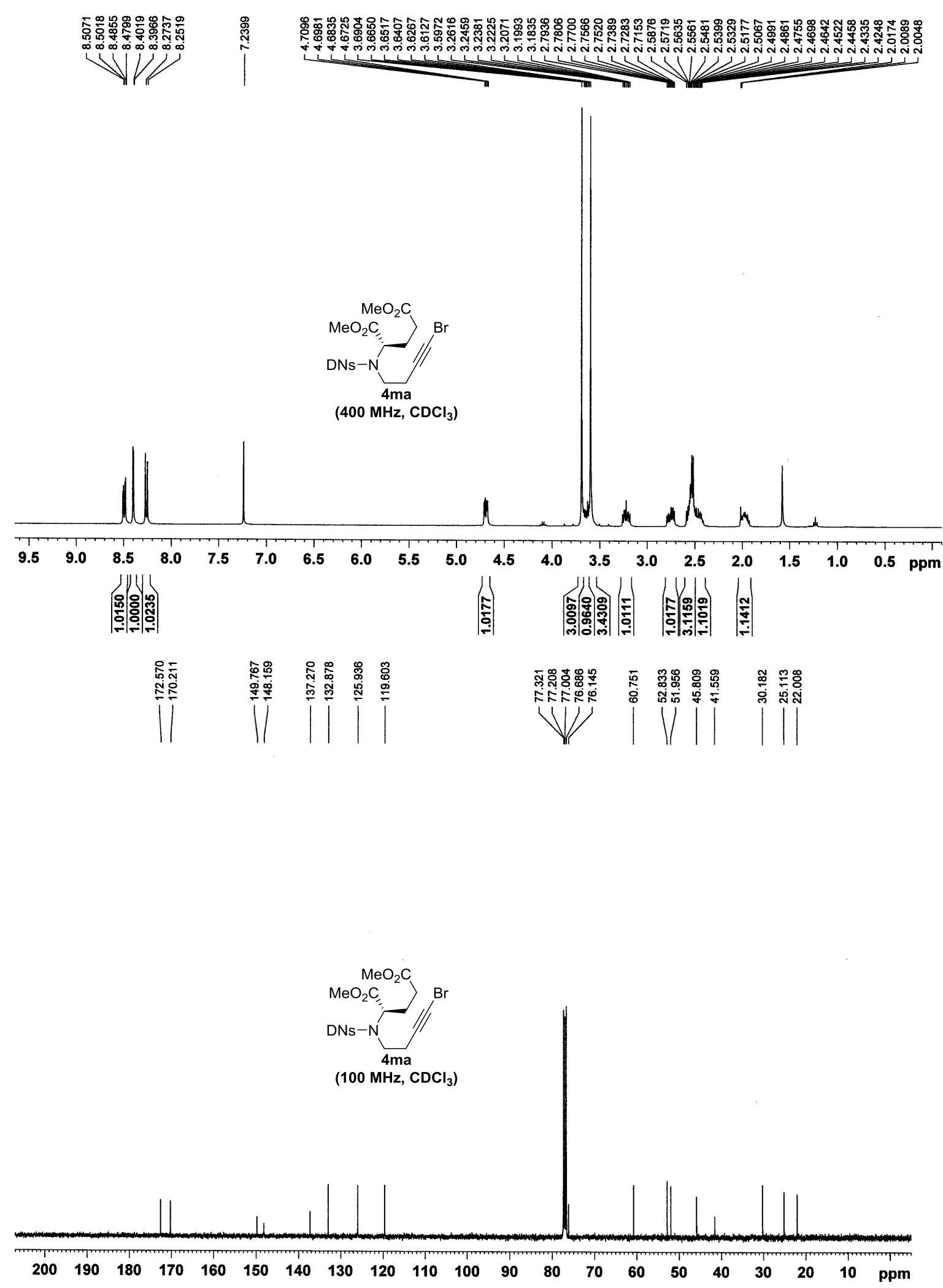




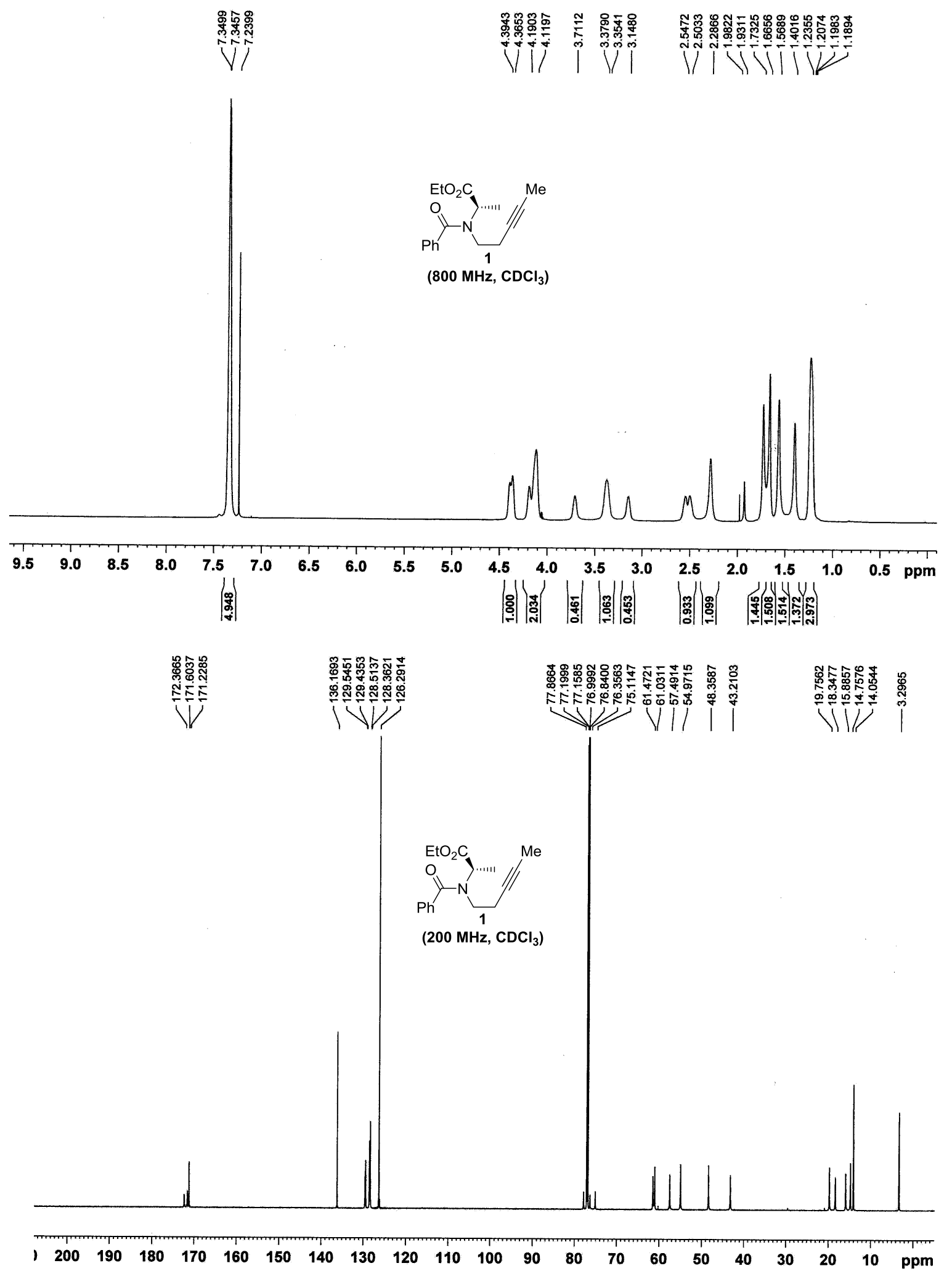



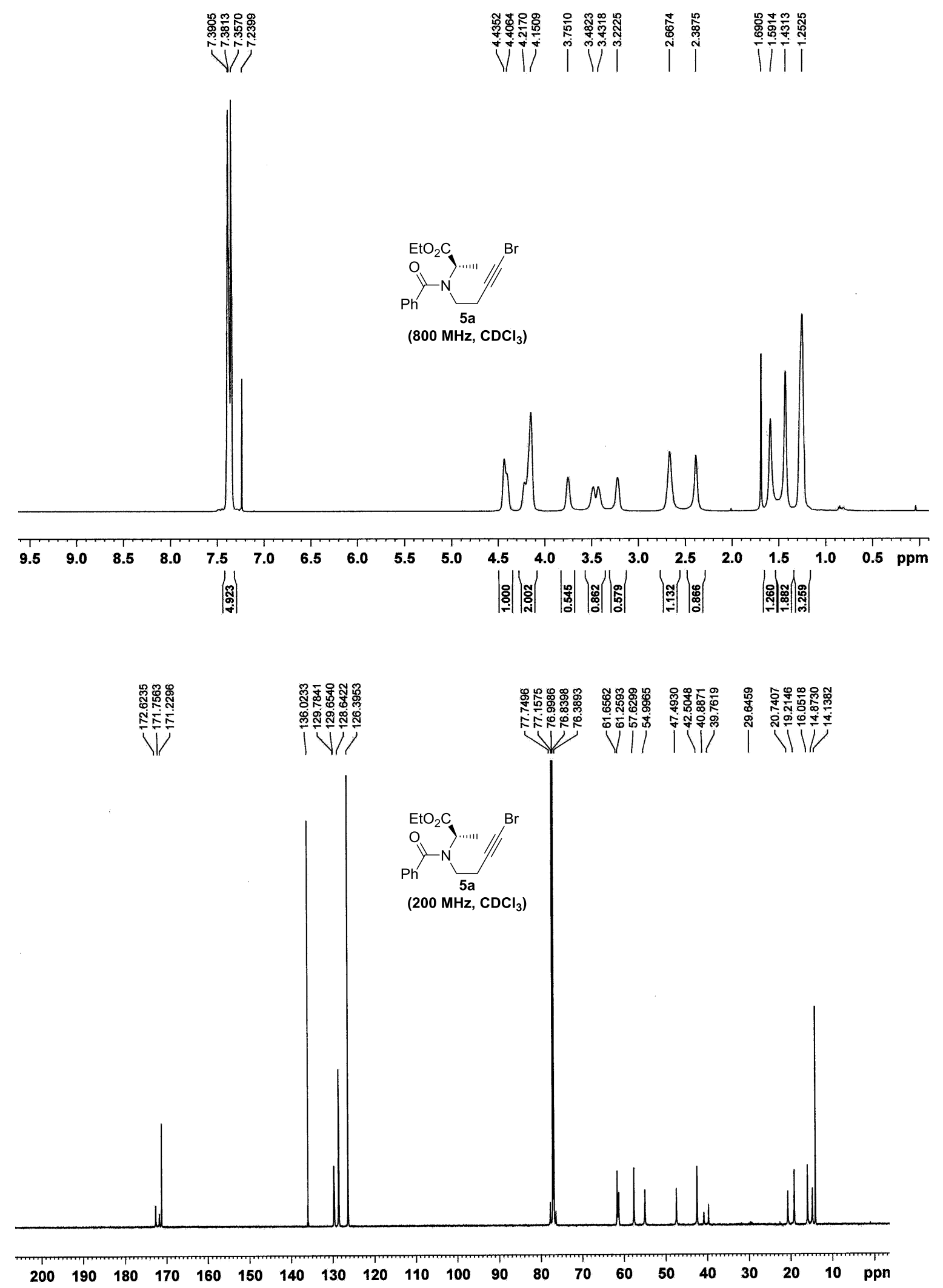


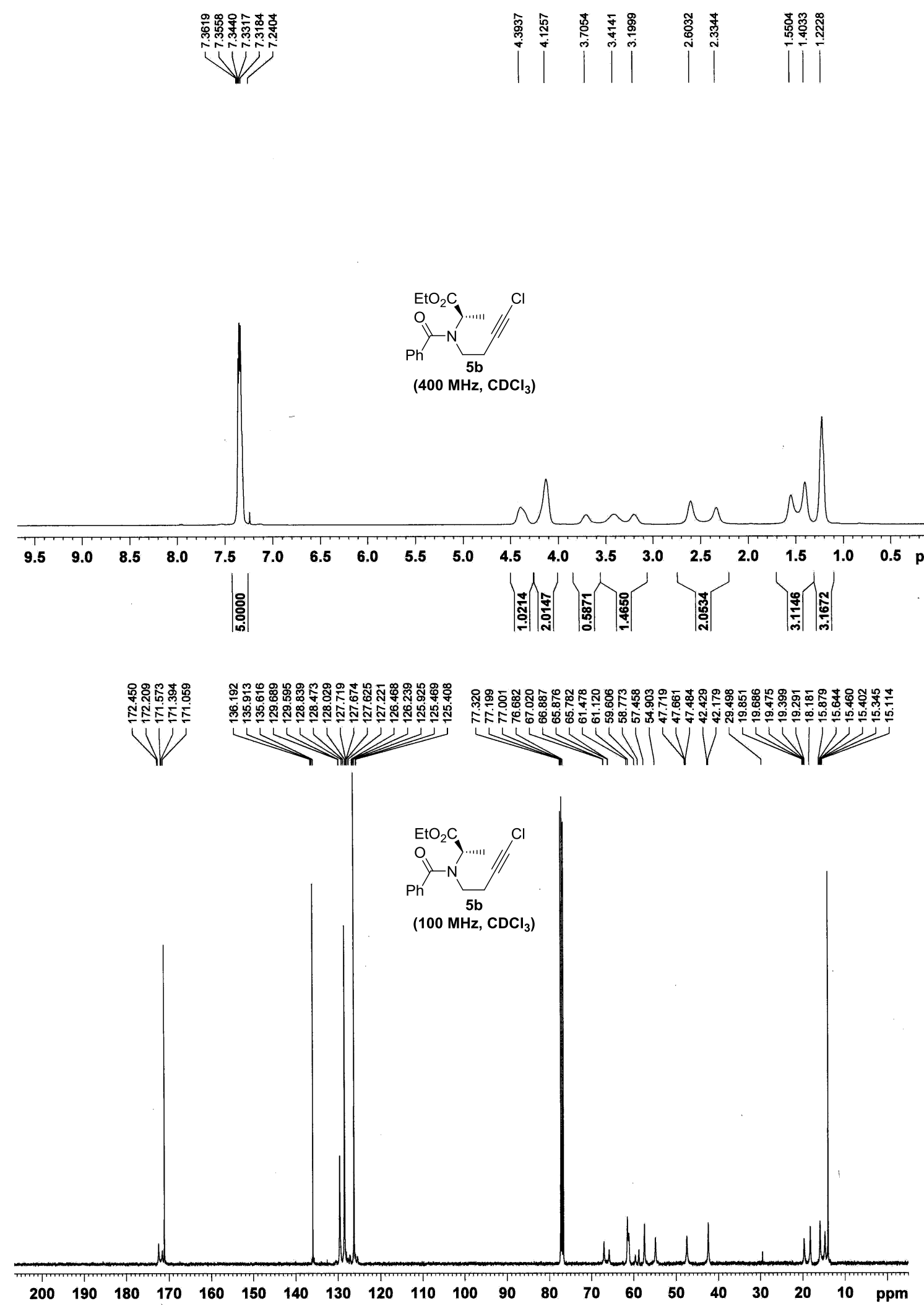



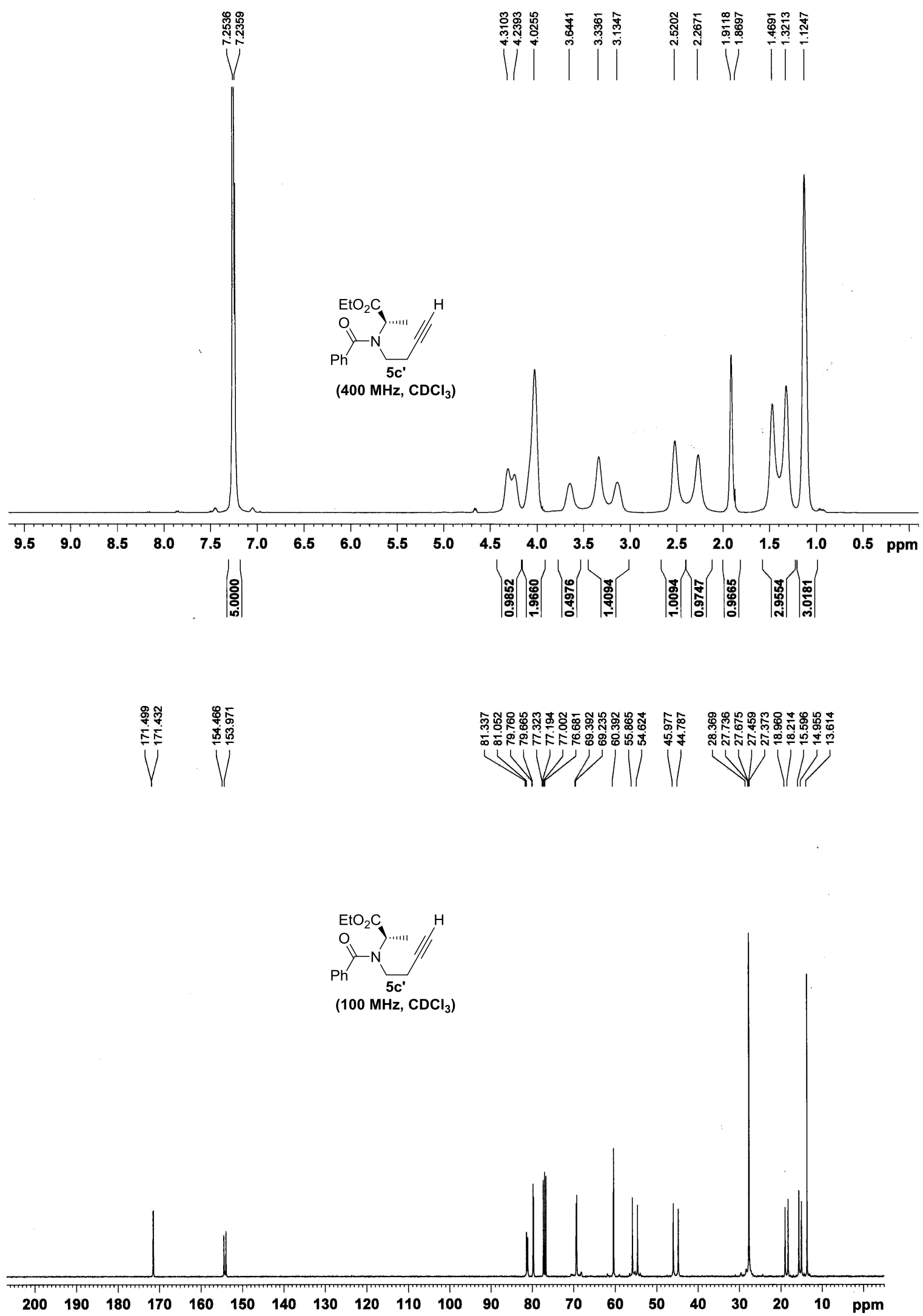

象

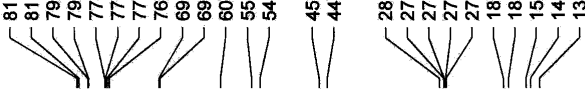




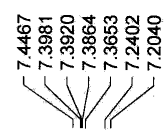

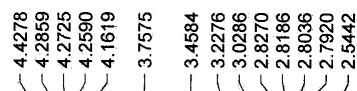

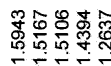

WI 1W1

ivi

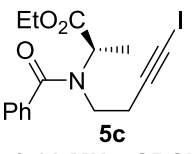

(500 MHz, $\mathrm{CDCl}_{3}$ )

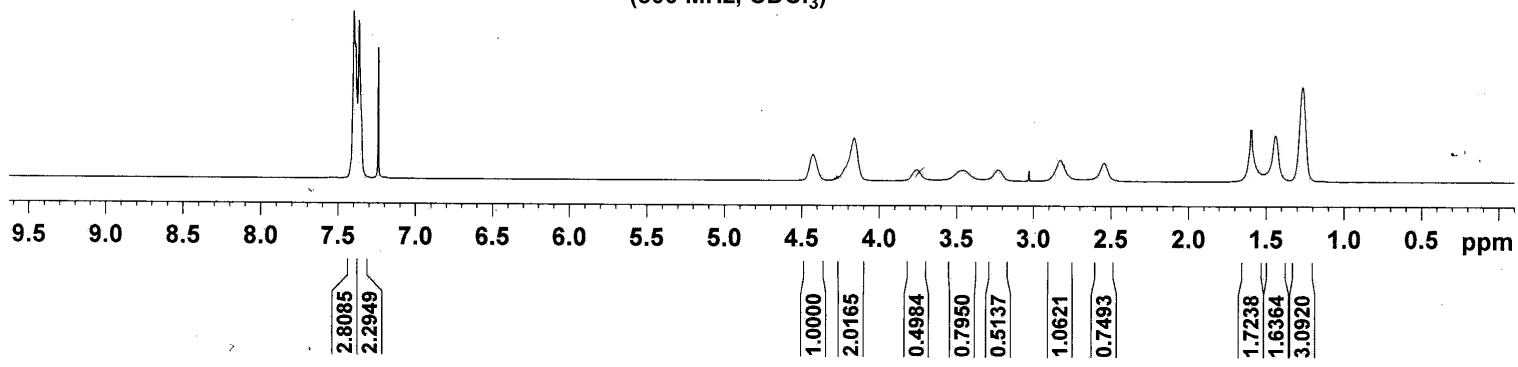

\begin{tabular}{|c|c|c|c|c|c|c|}
\hline 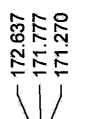 & 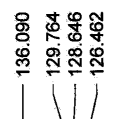 & कृष & 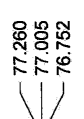 & 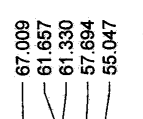 & 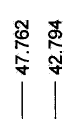 & | \\
\hline
\end{tabular}

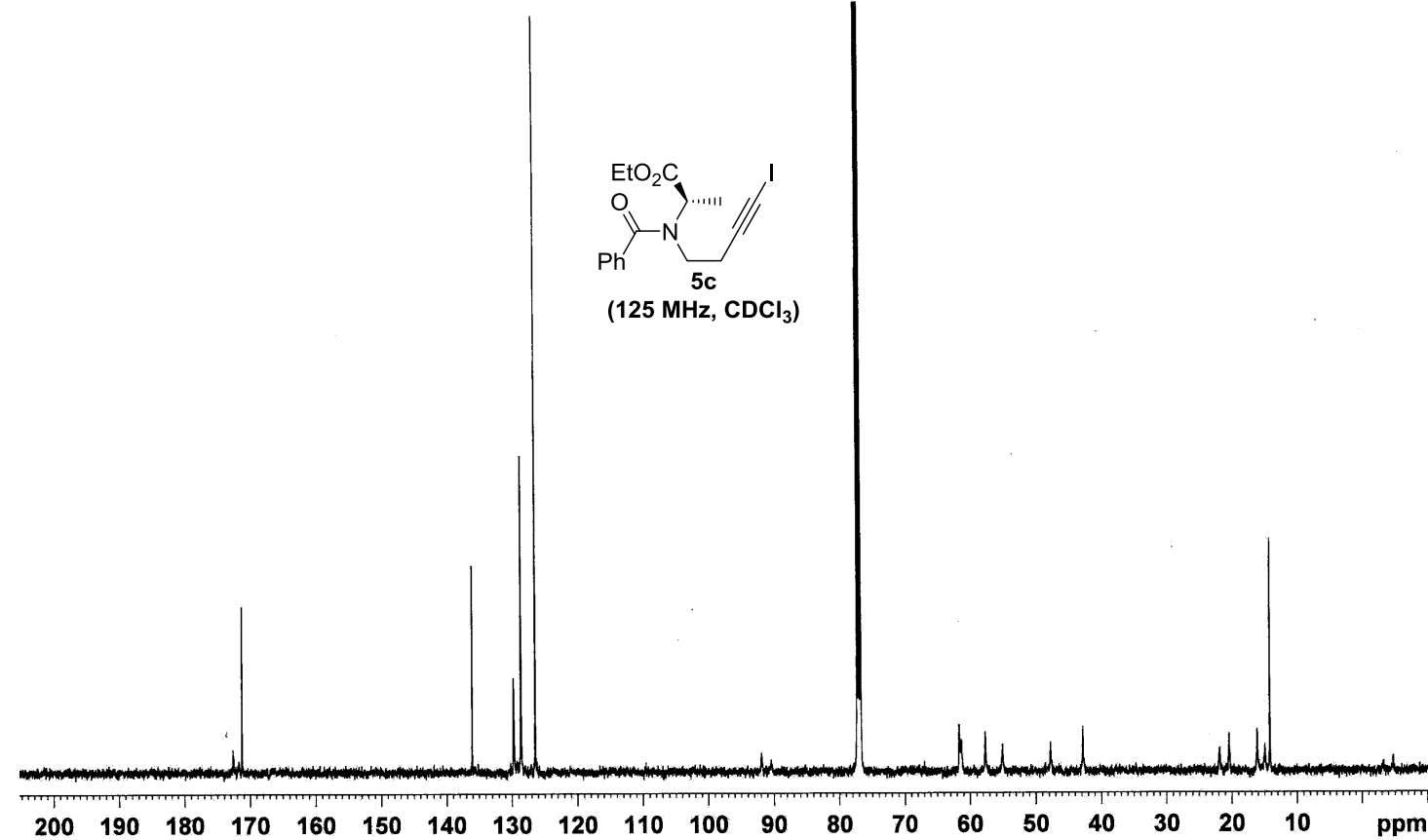




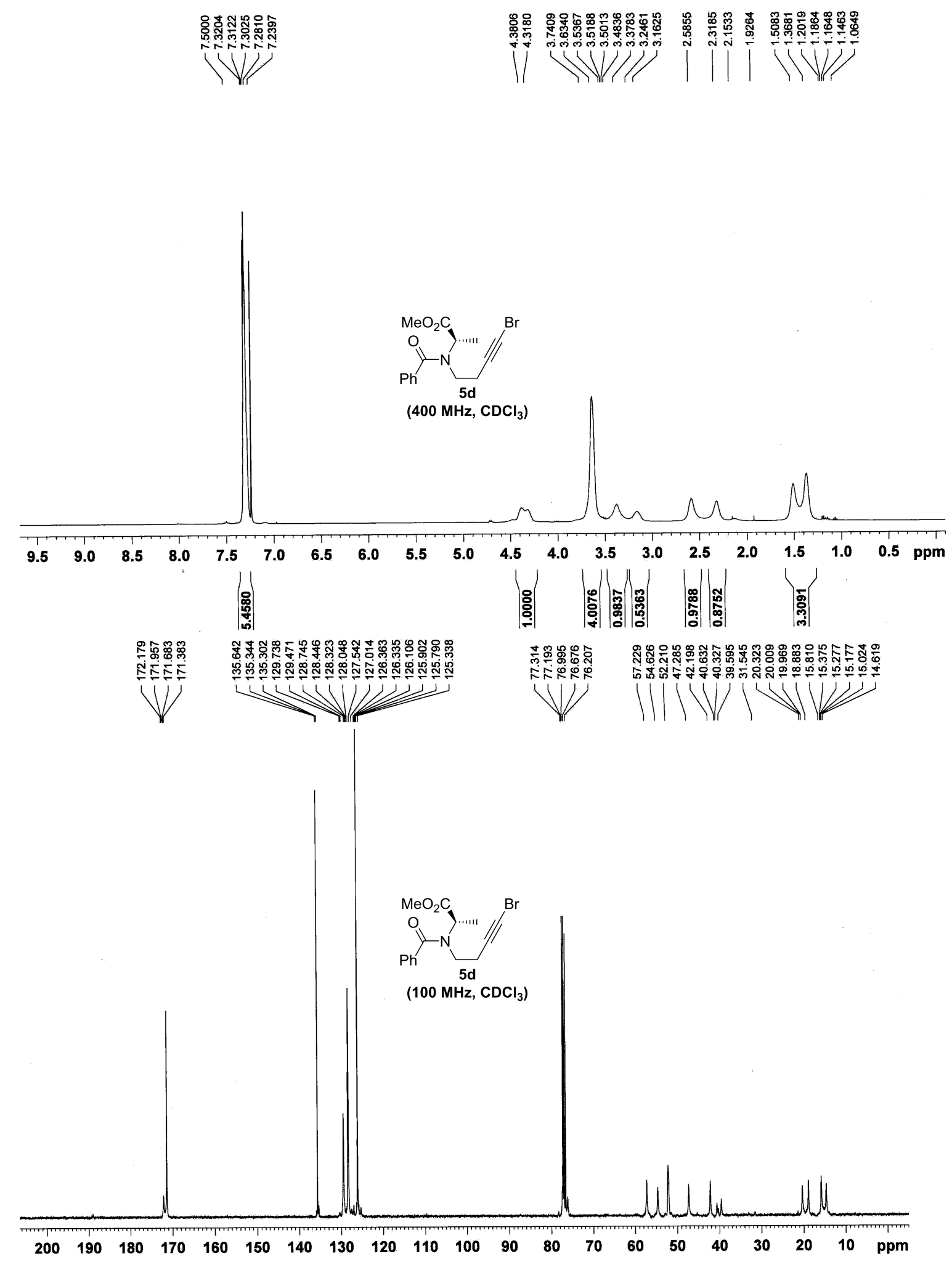




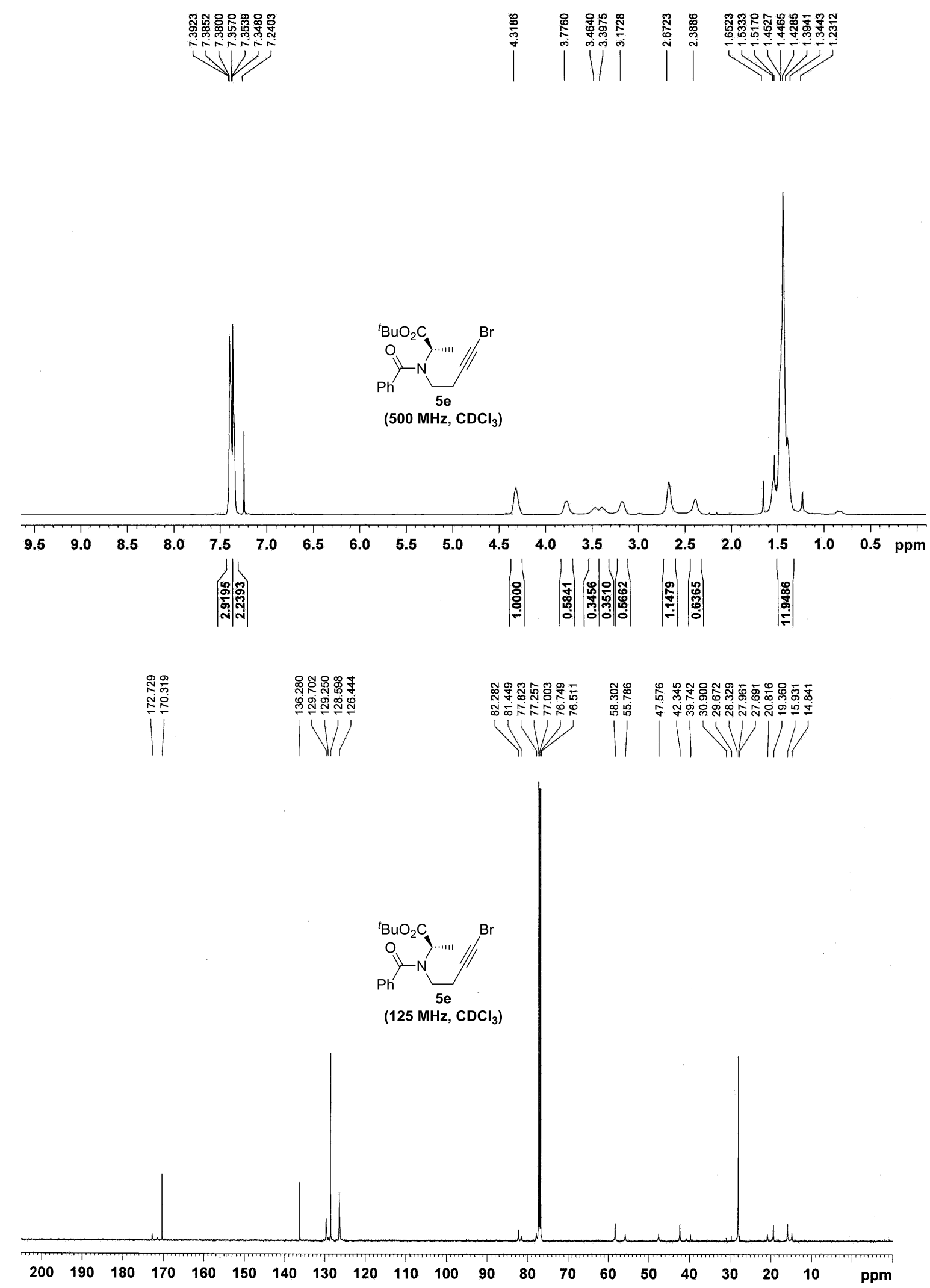




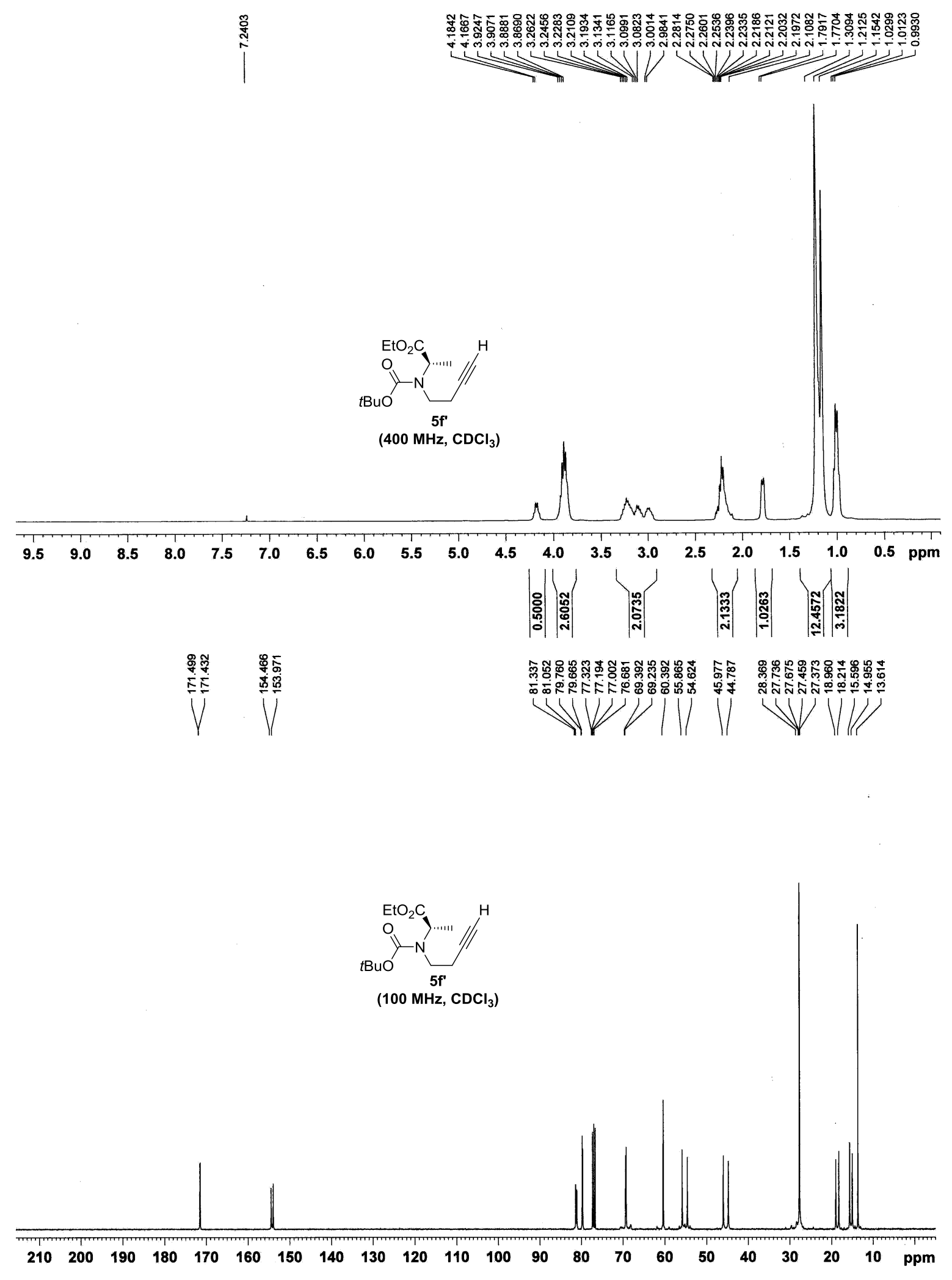



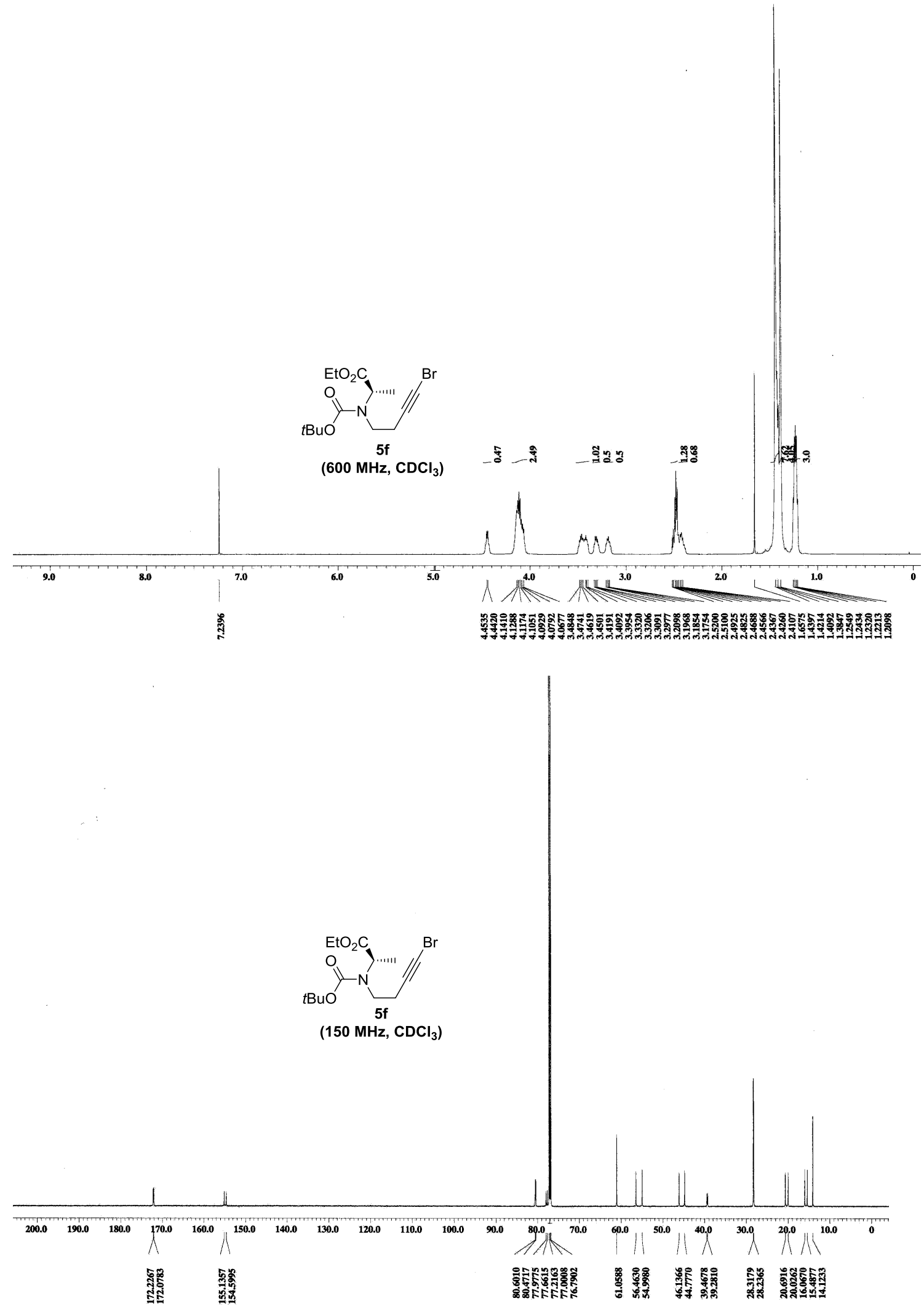

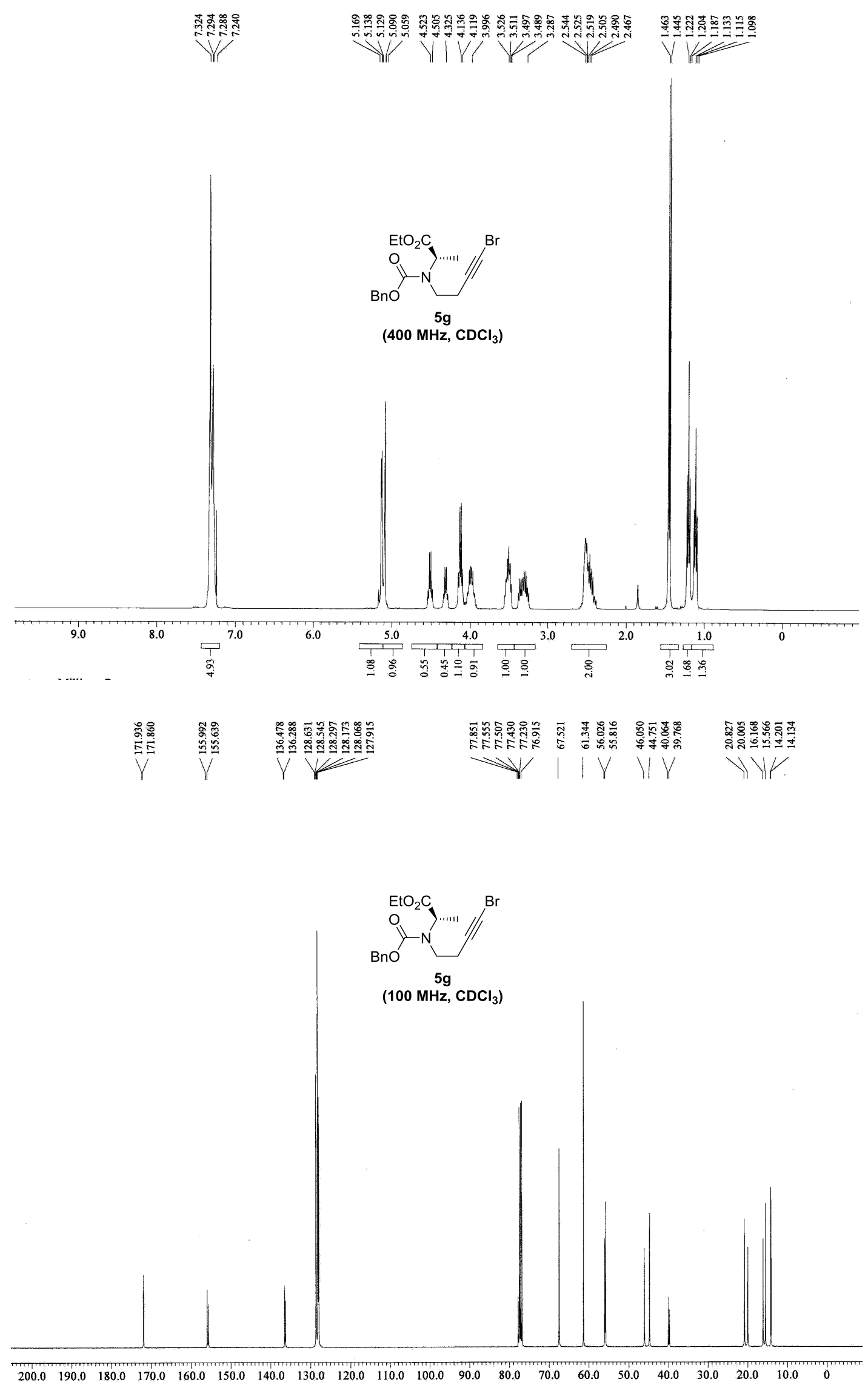


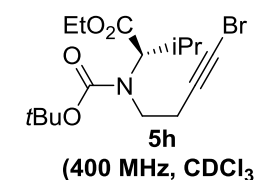

(400 $\mathrm{MHz}, \mathrm{CDCl}_{3}$ )
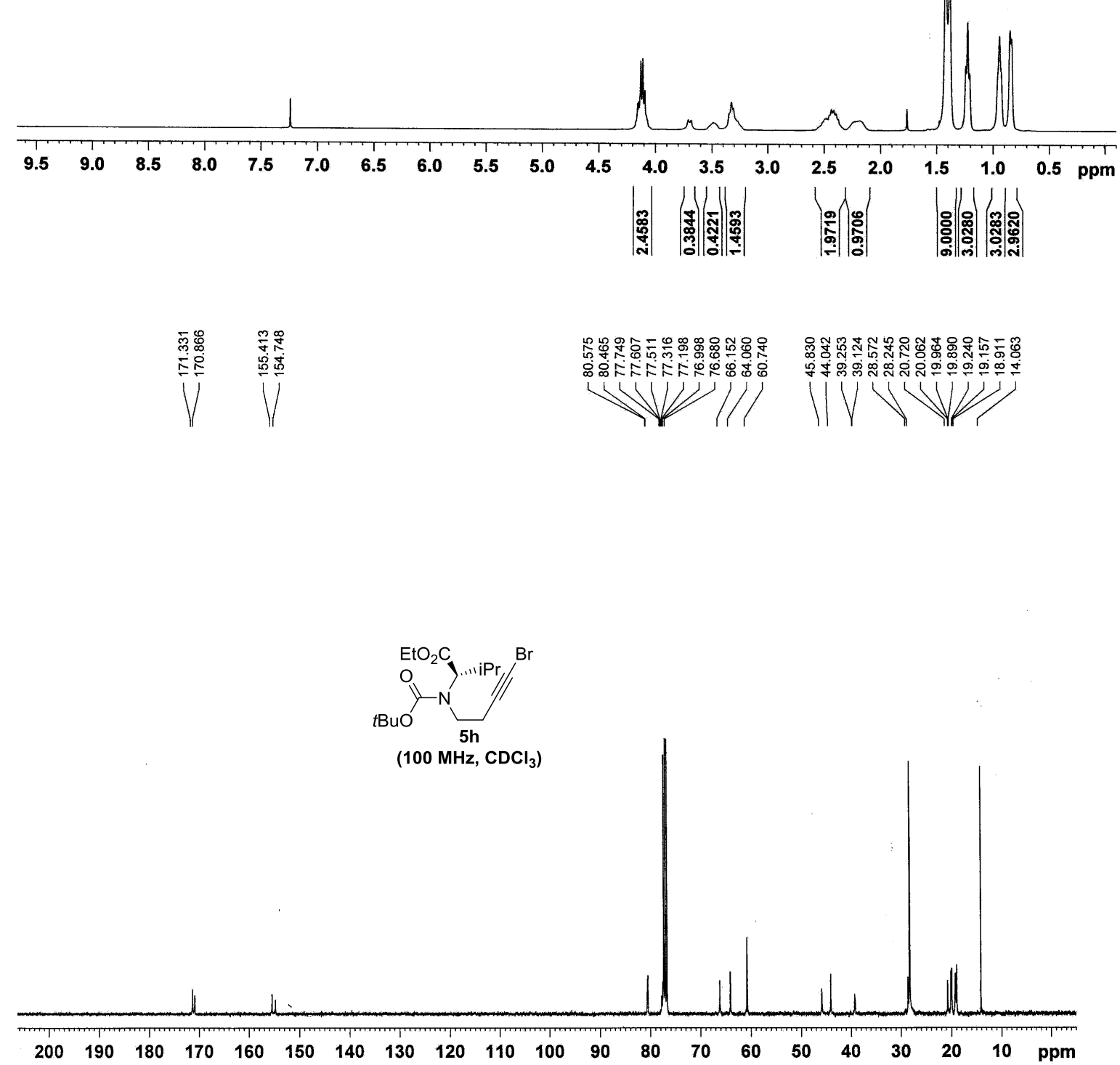


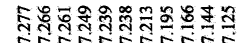

Nirnent

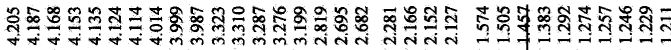
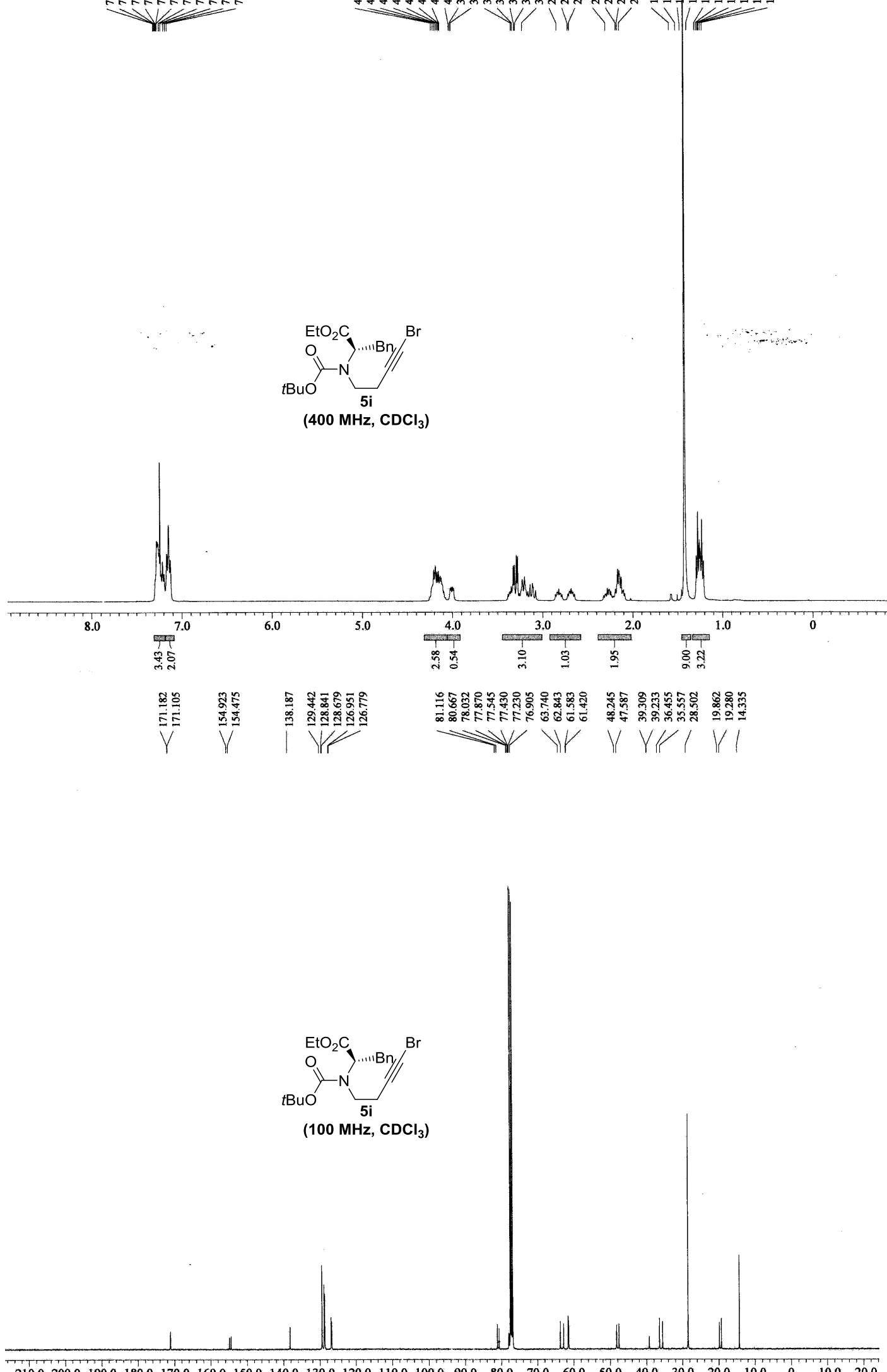


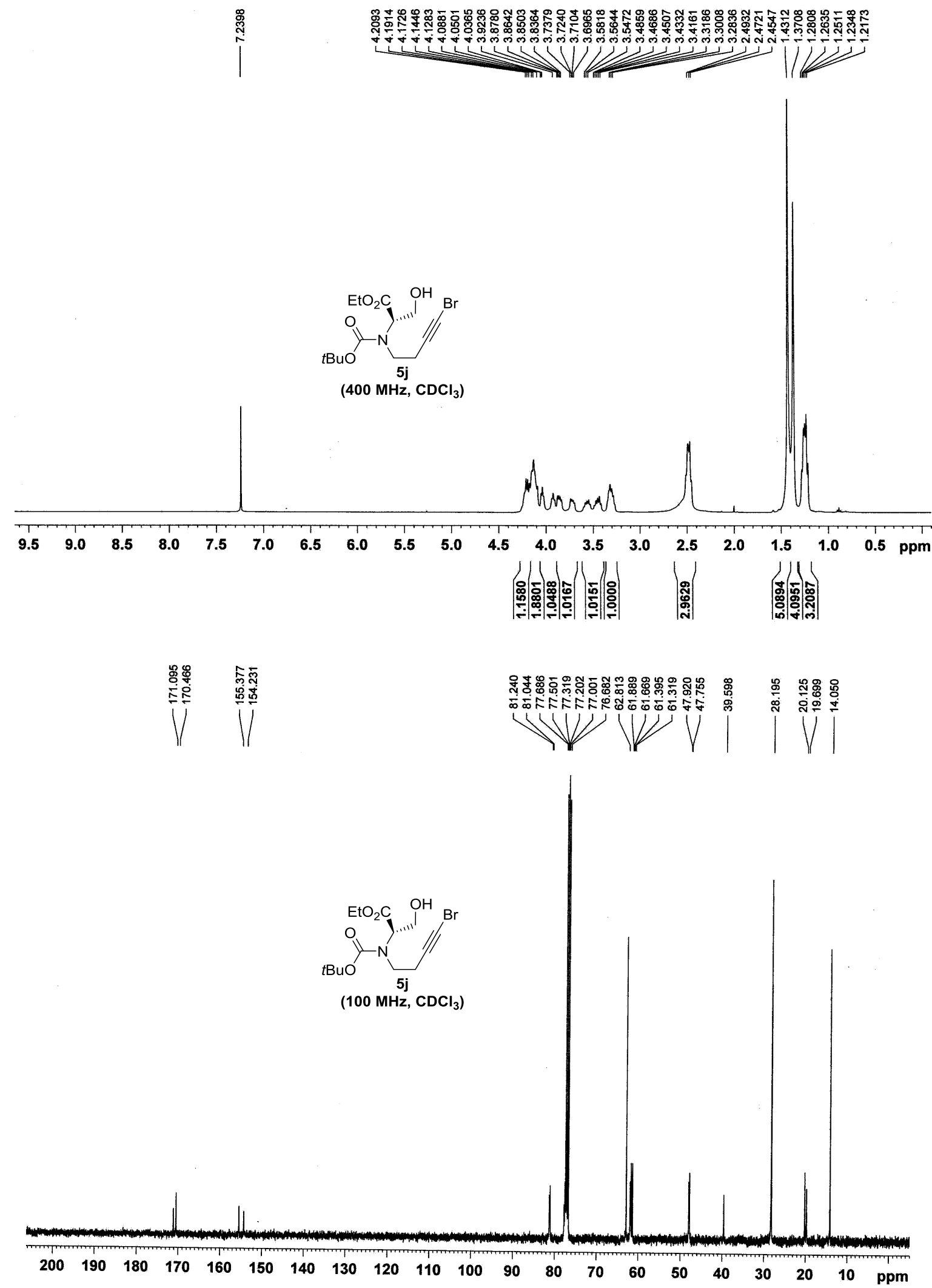




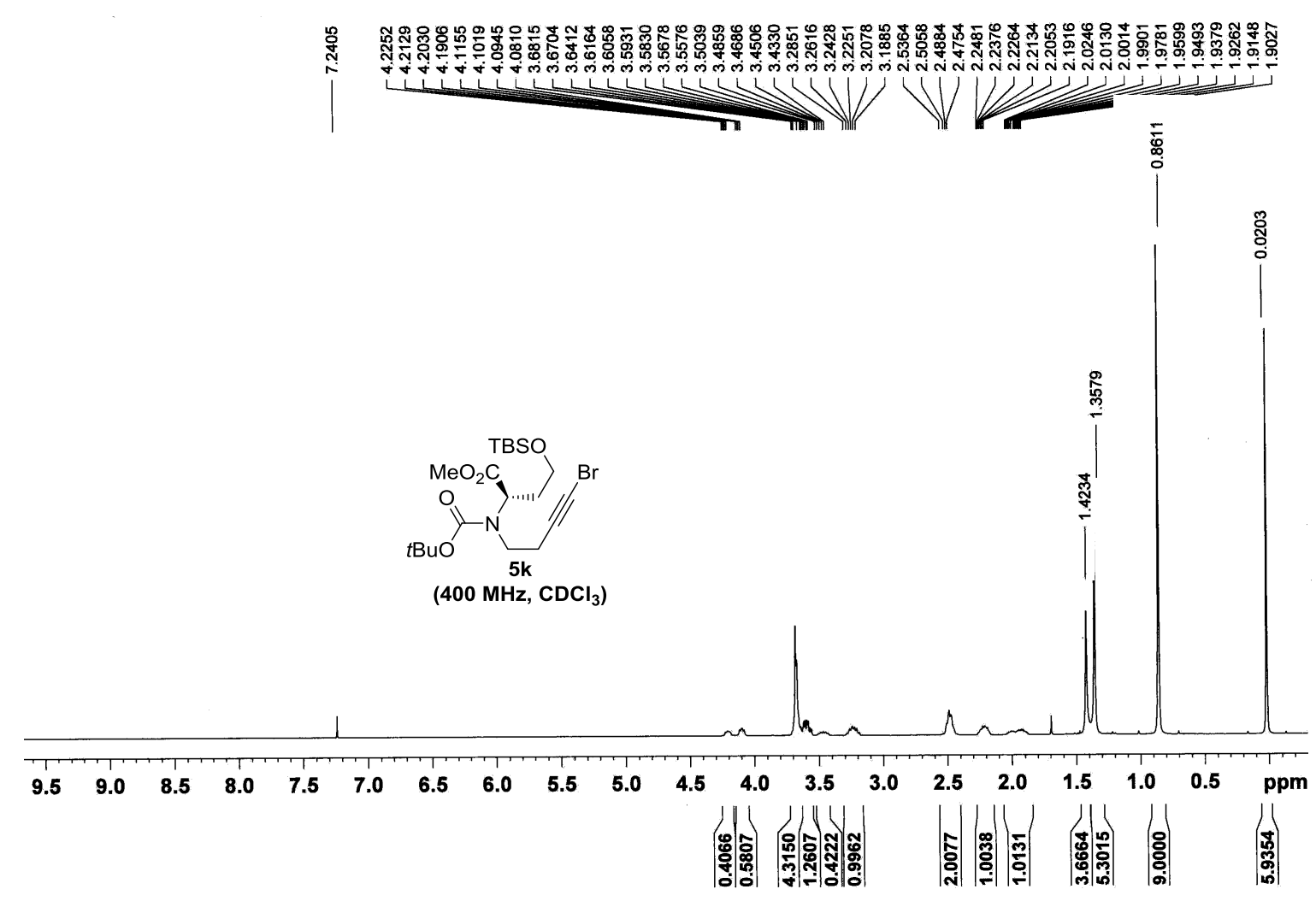

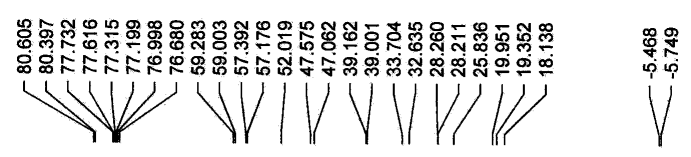

$\int^{\frac{9}{9}}$

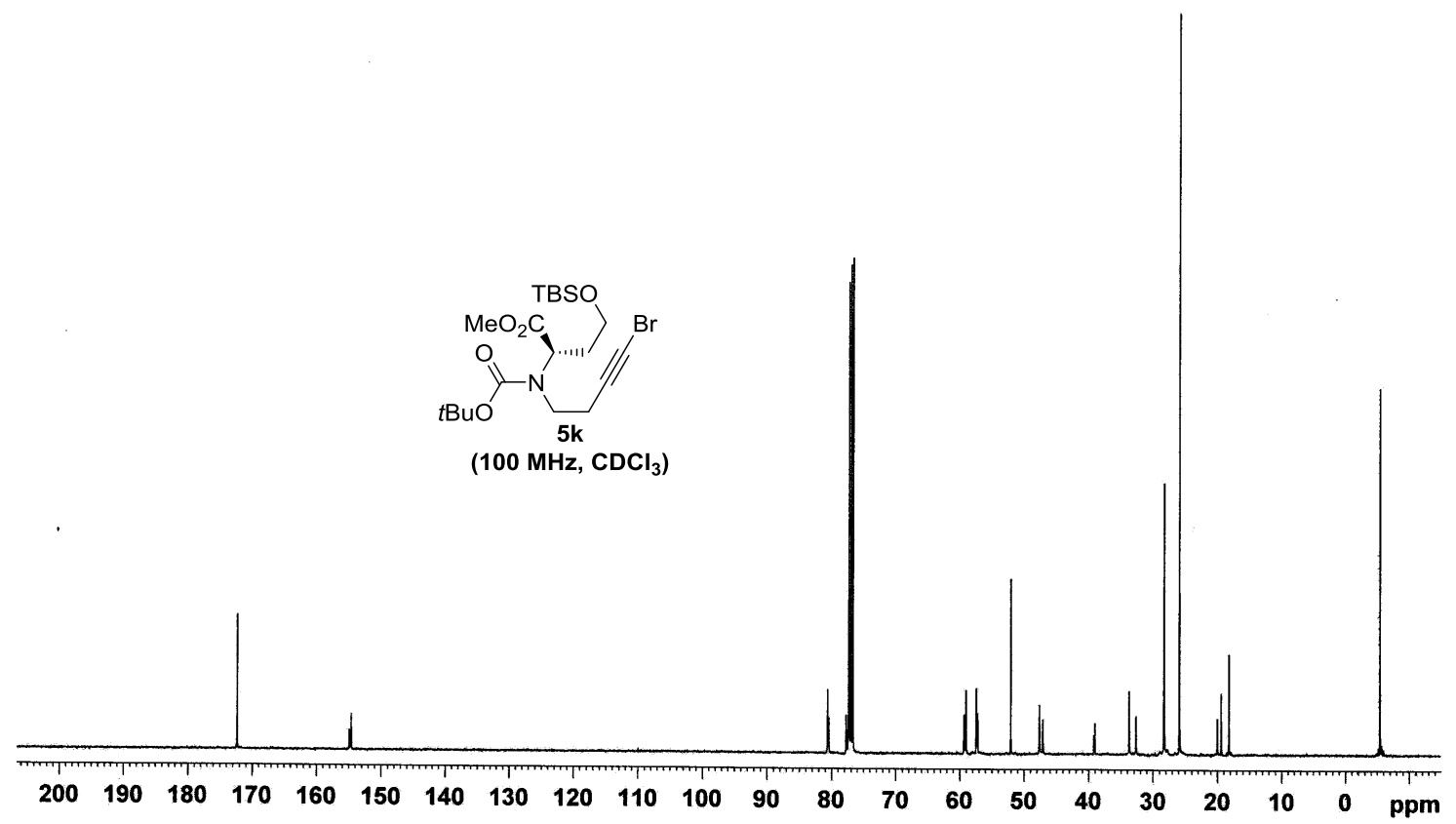




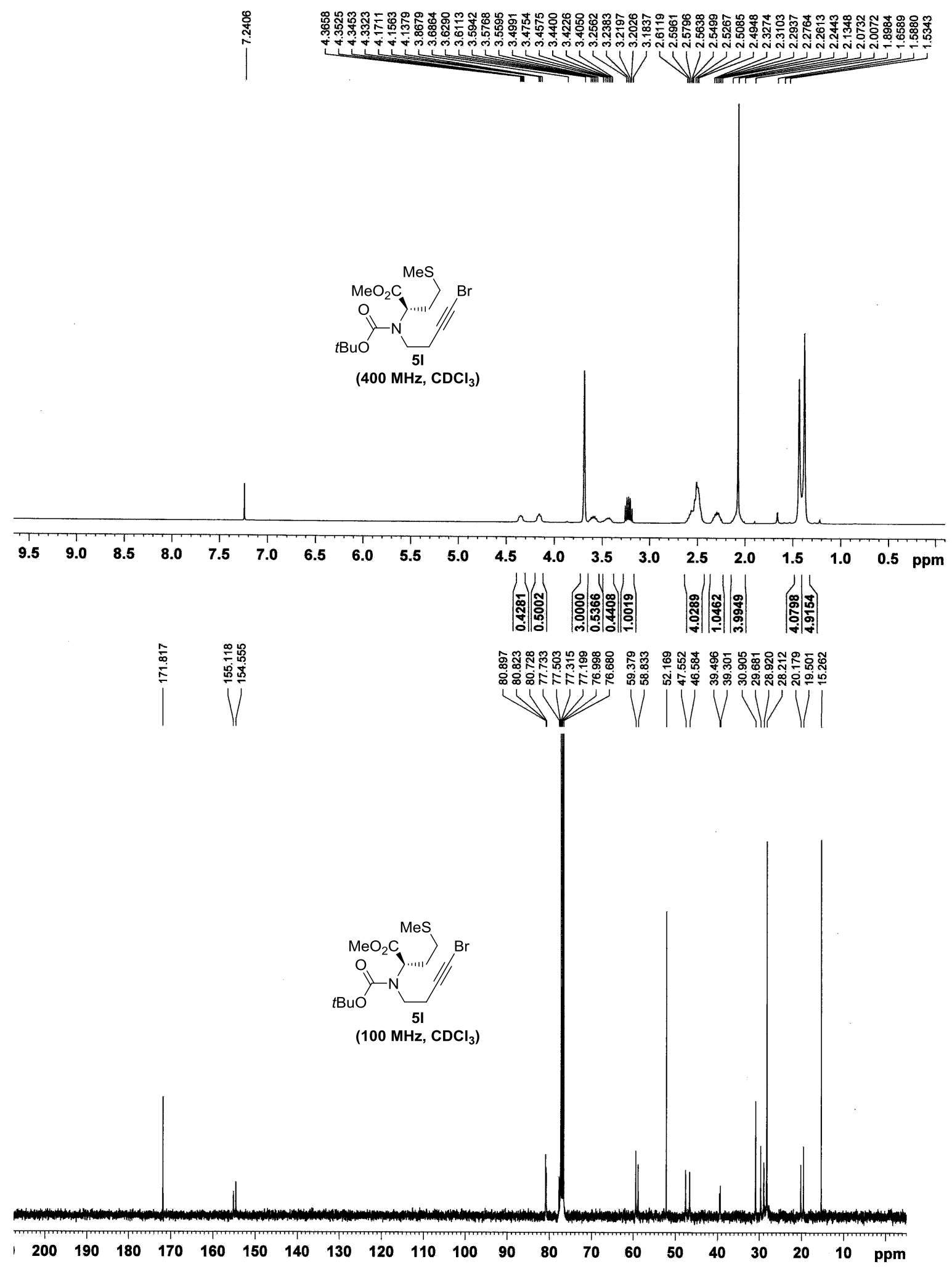




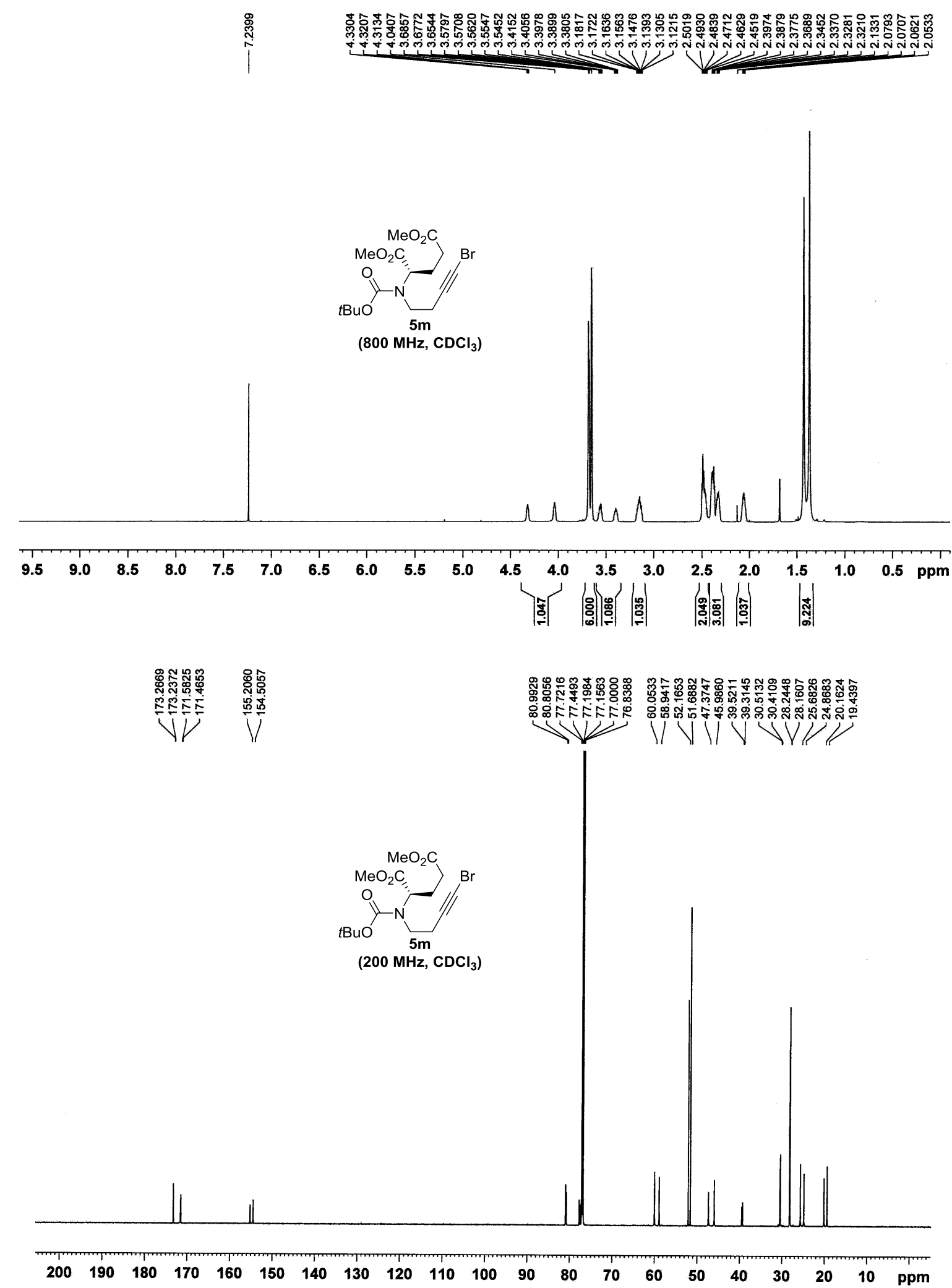



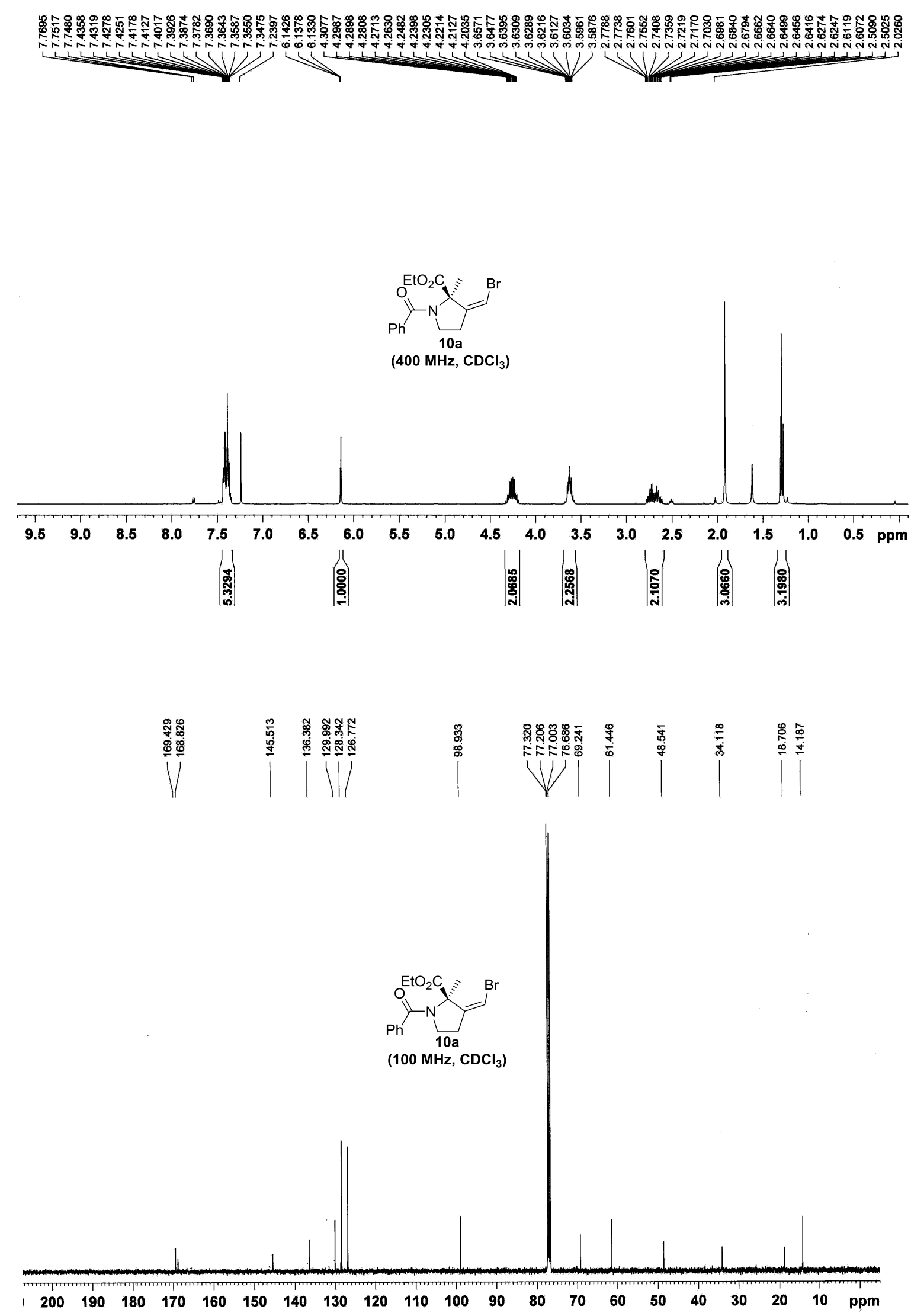


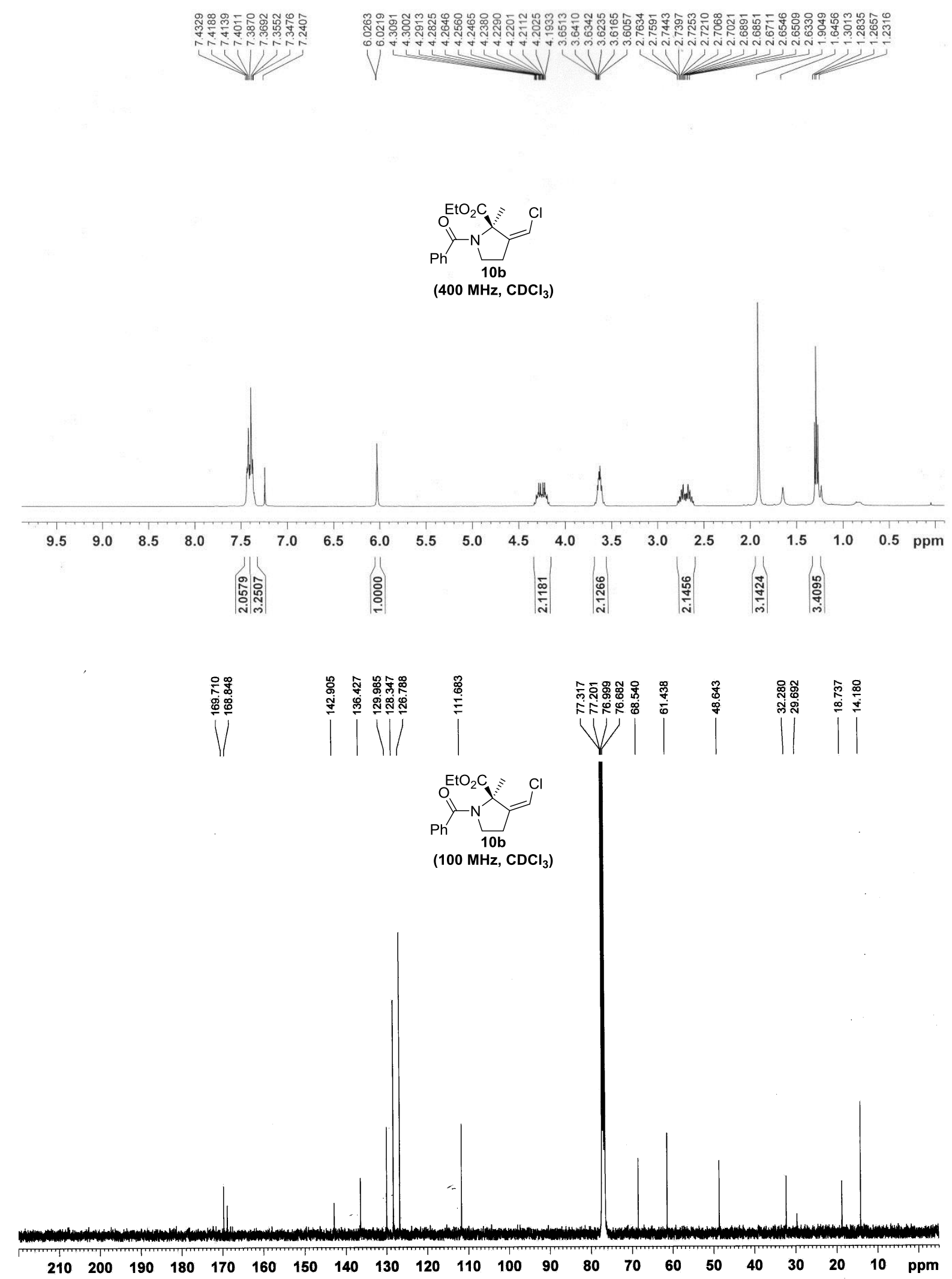



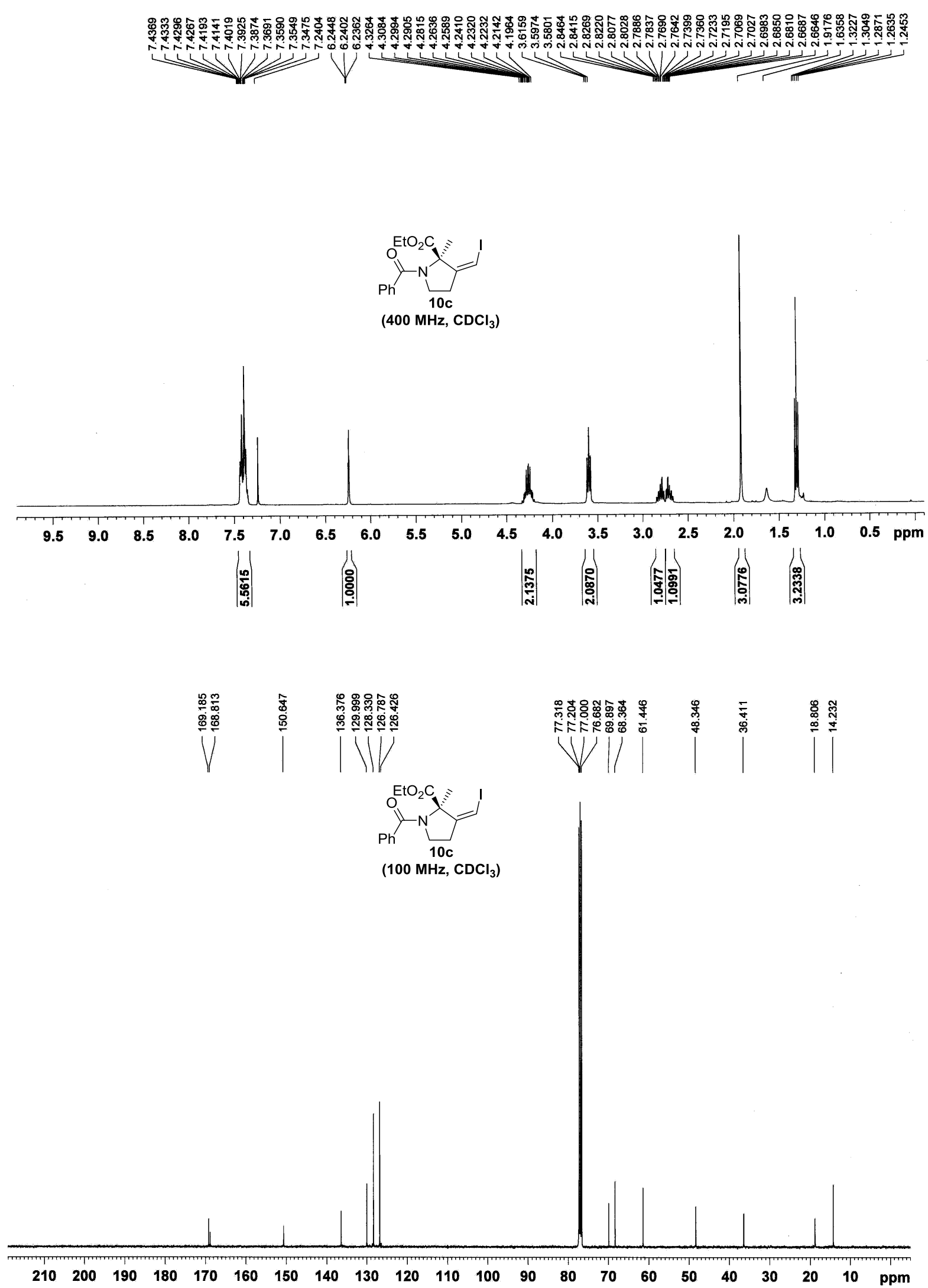

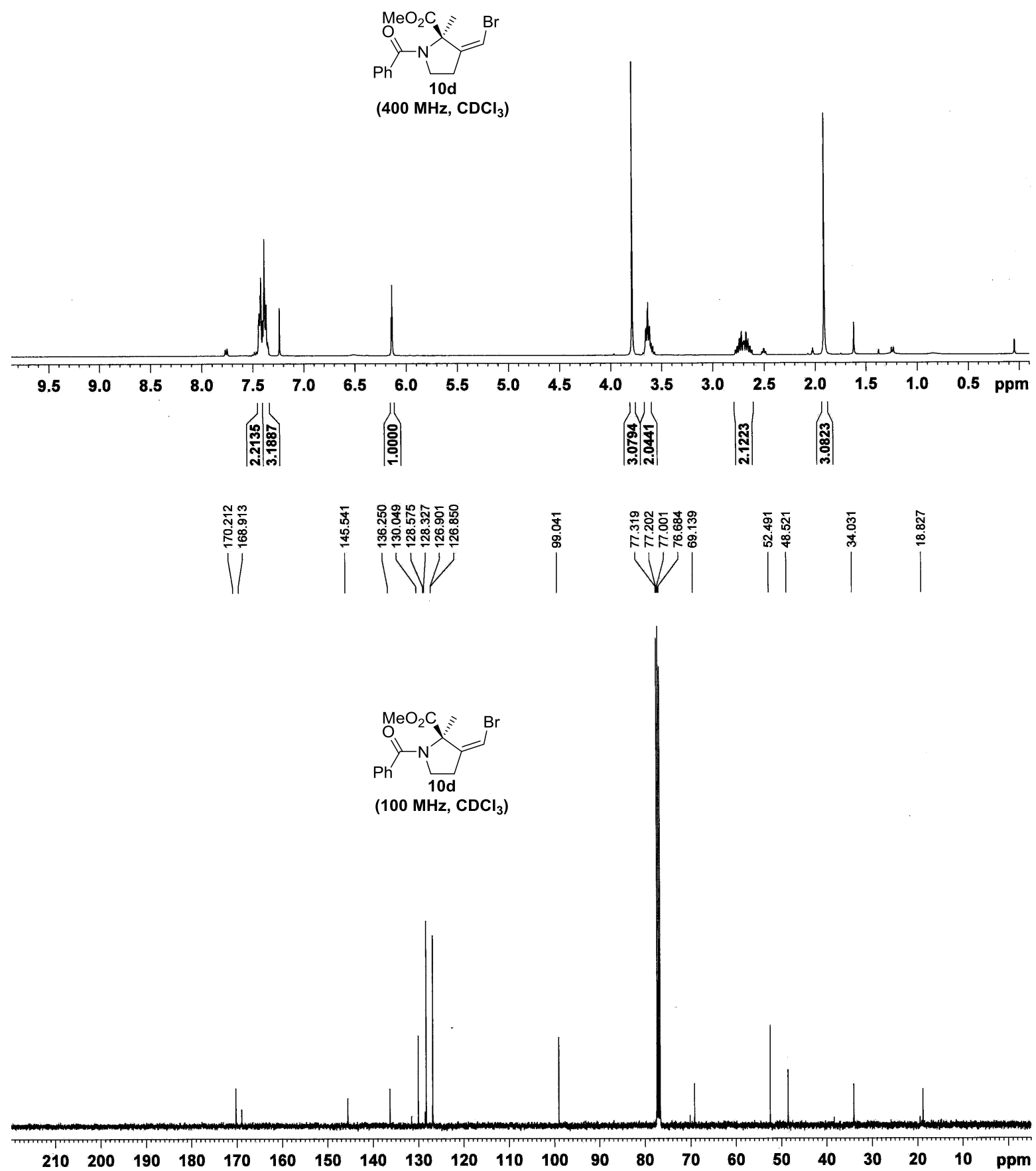


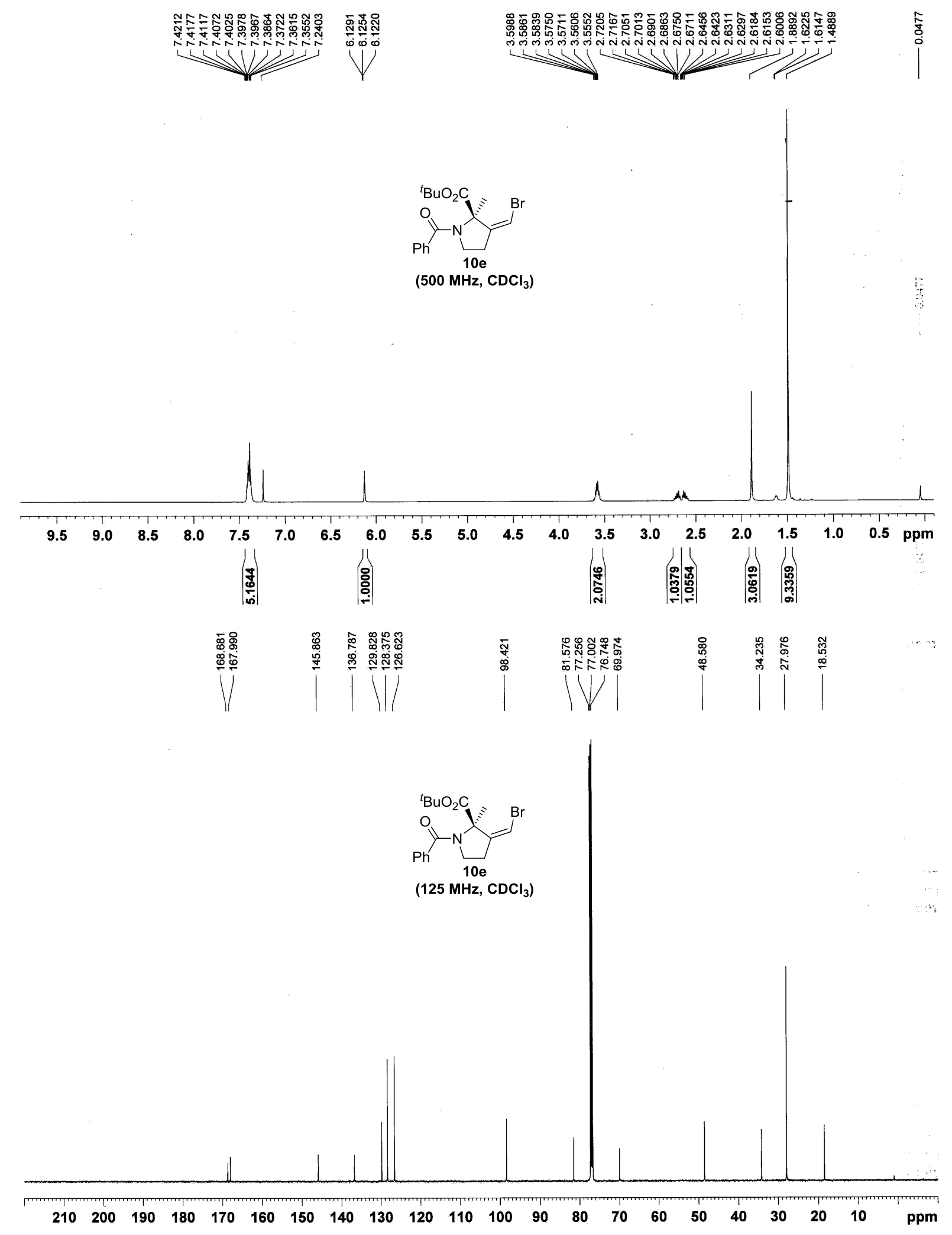




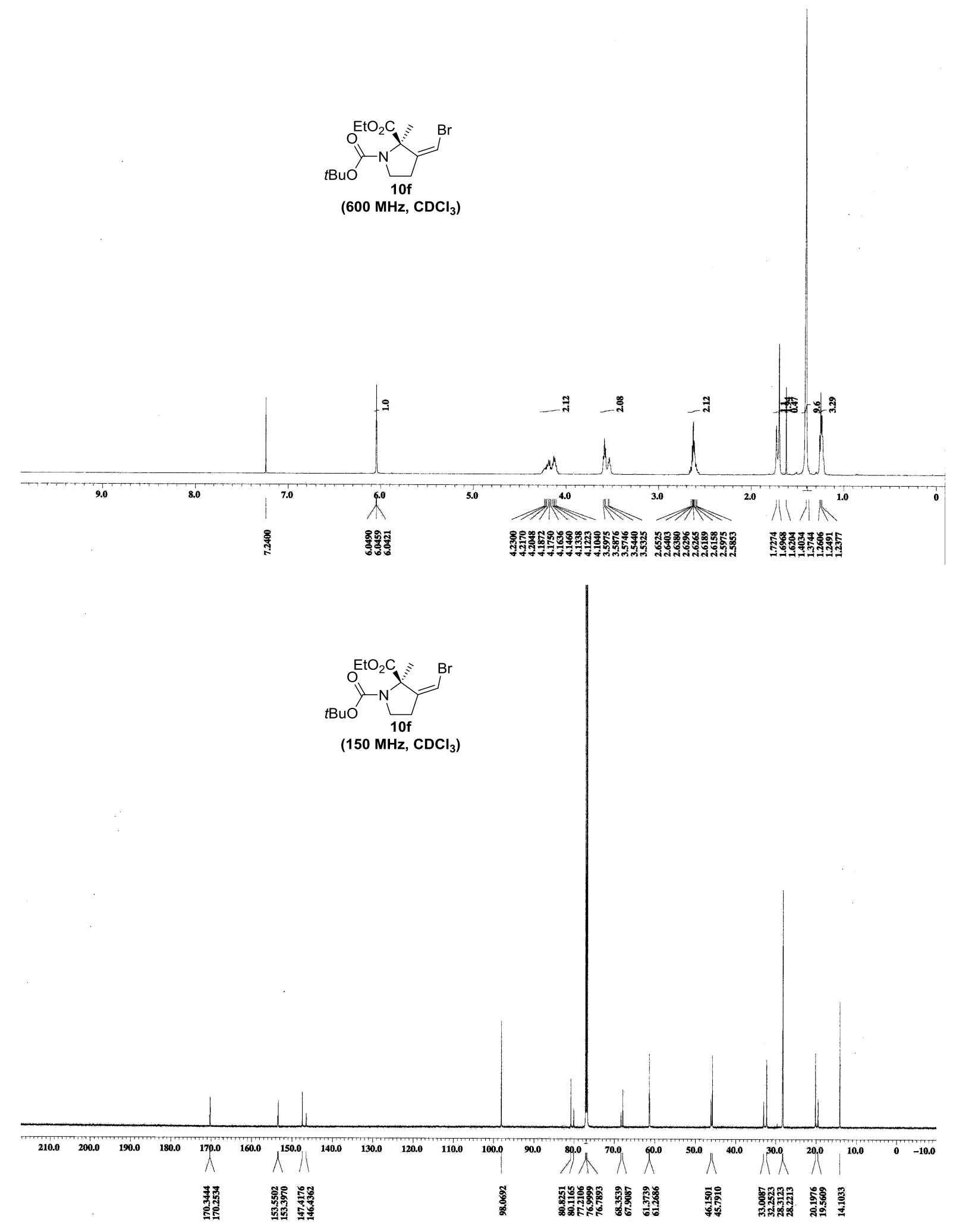



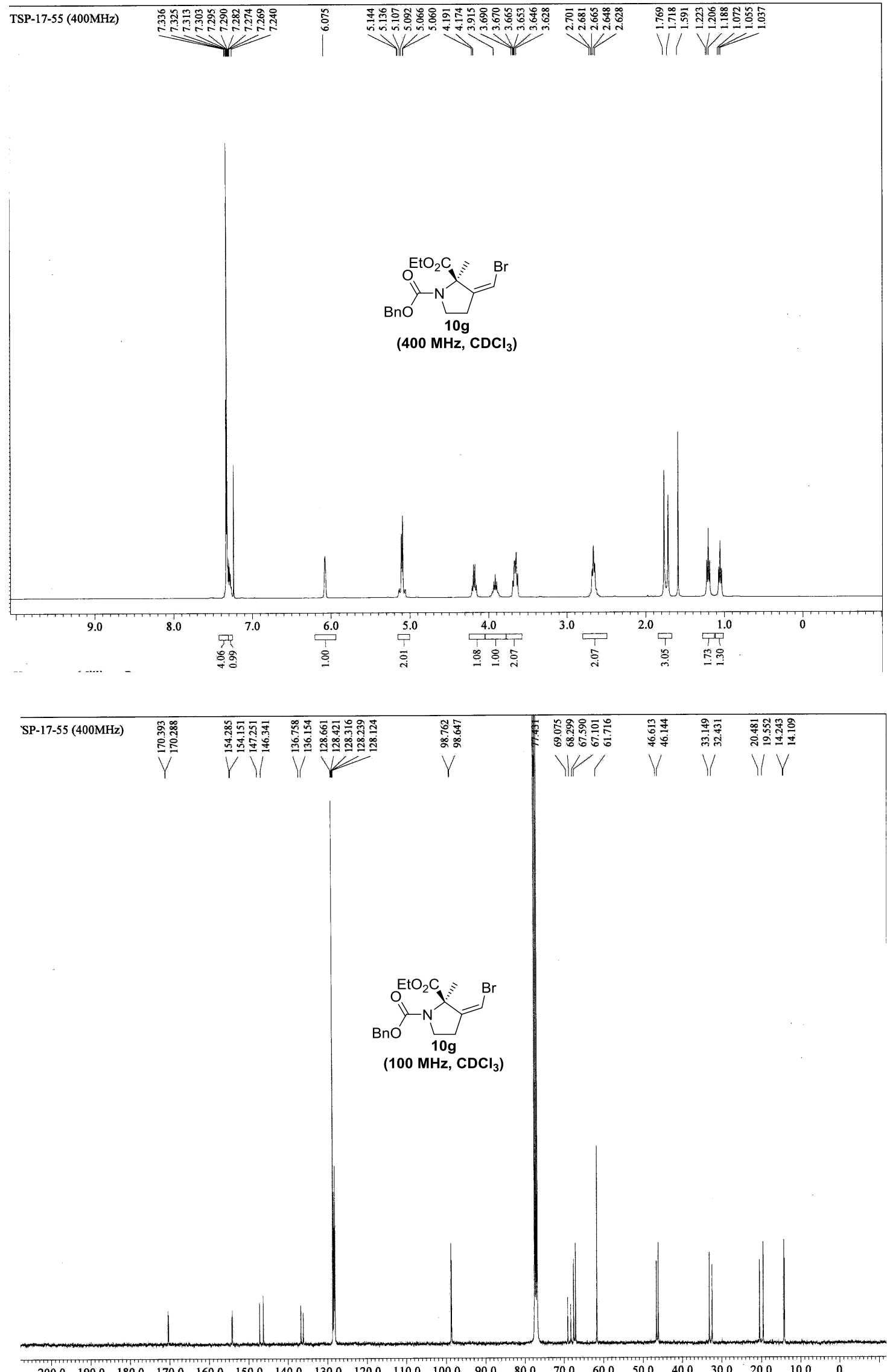

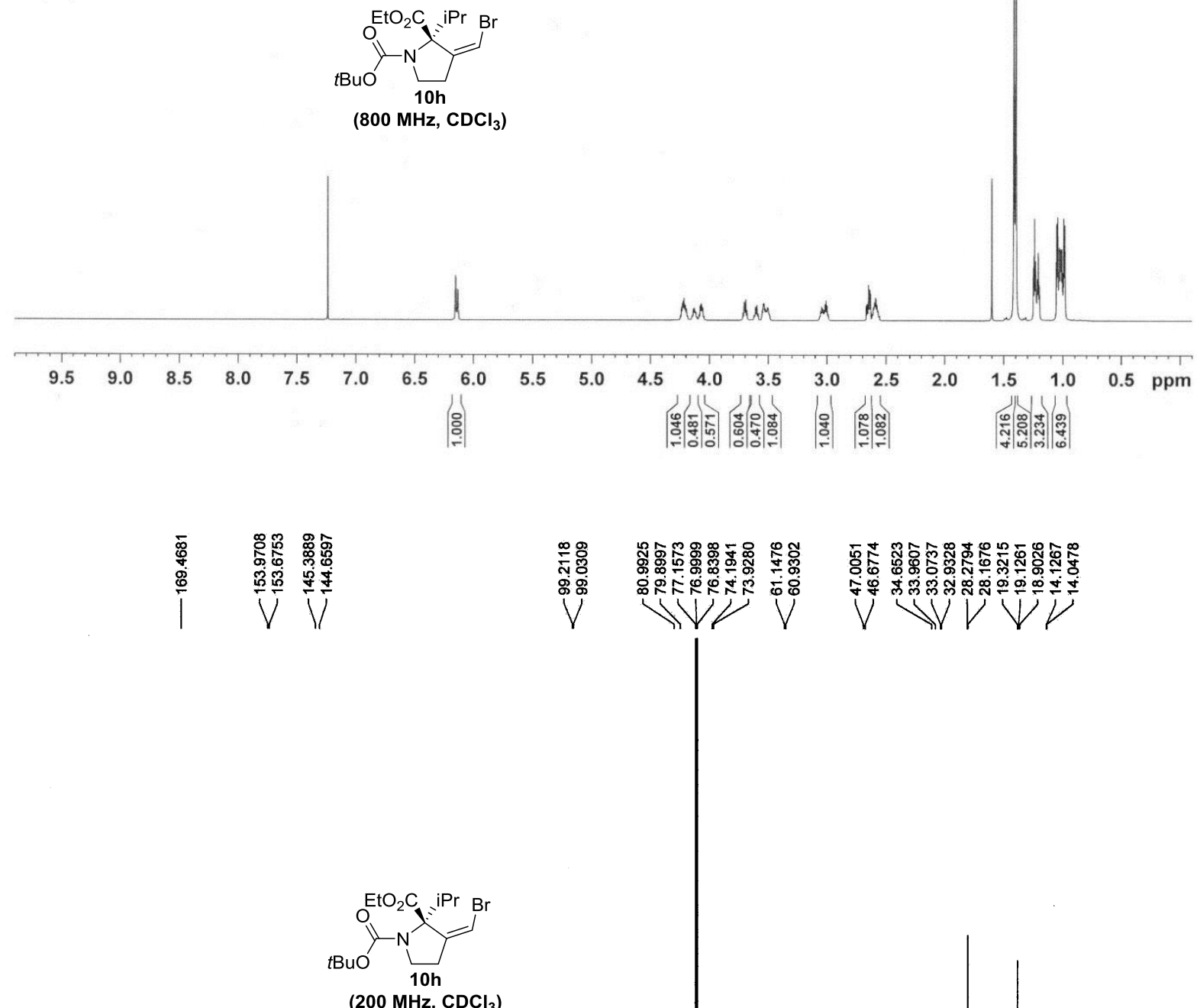

(200 MHz, $\mathrm{CDCl}_{3}$ )
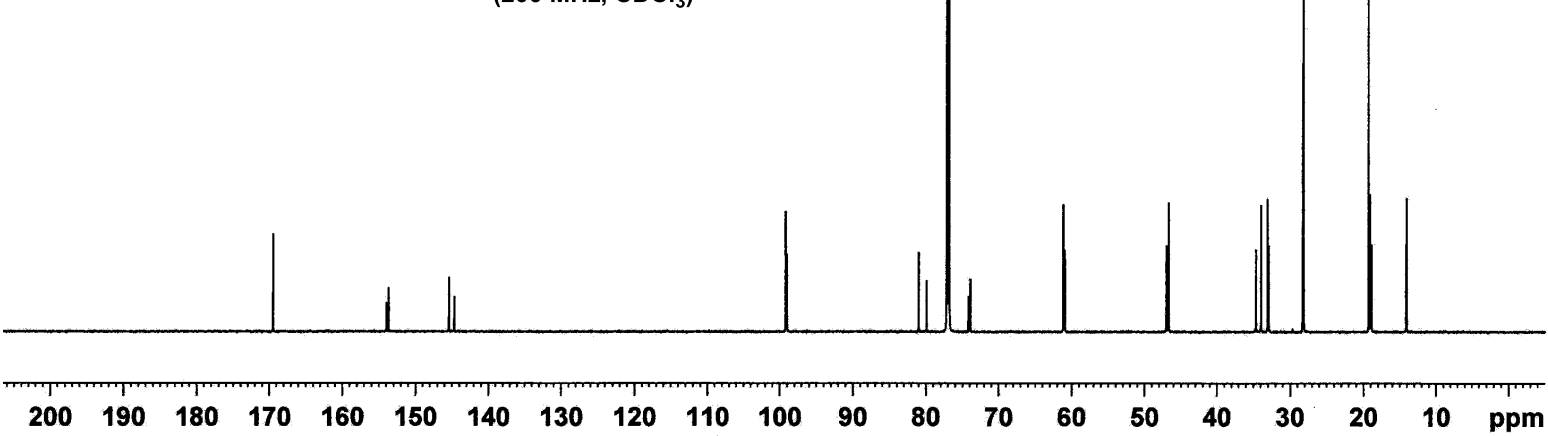


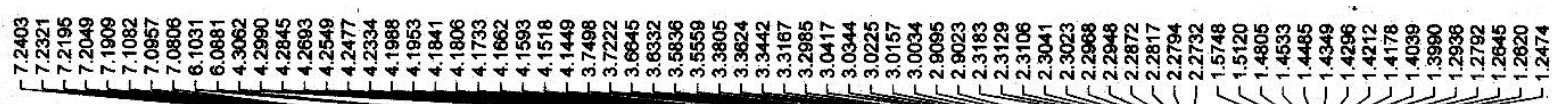

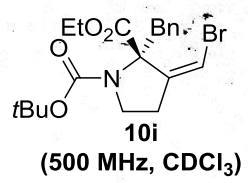
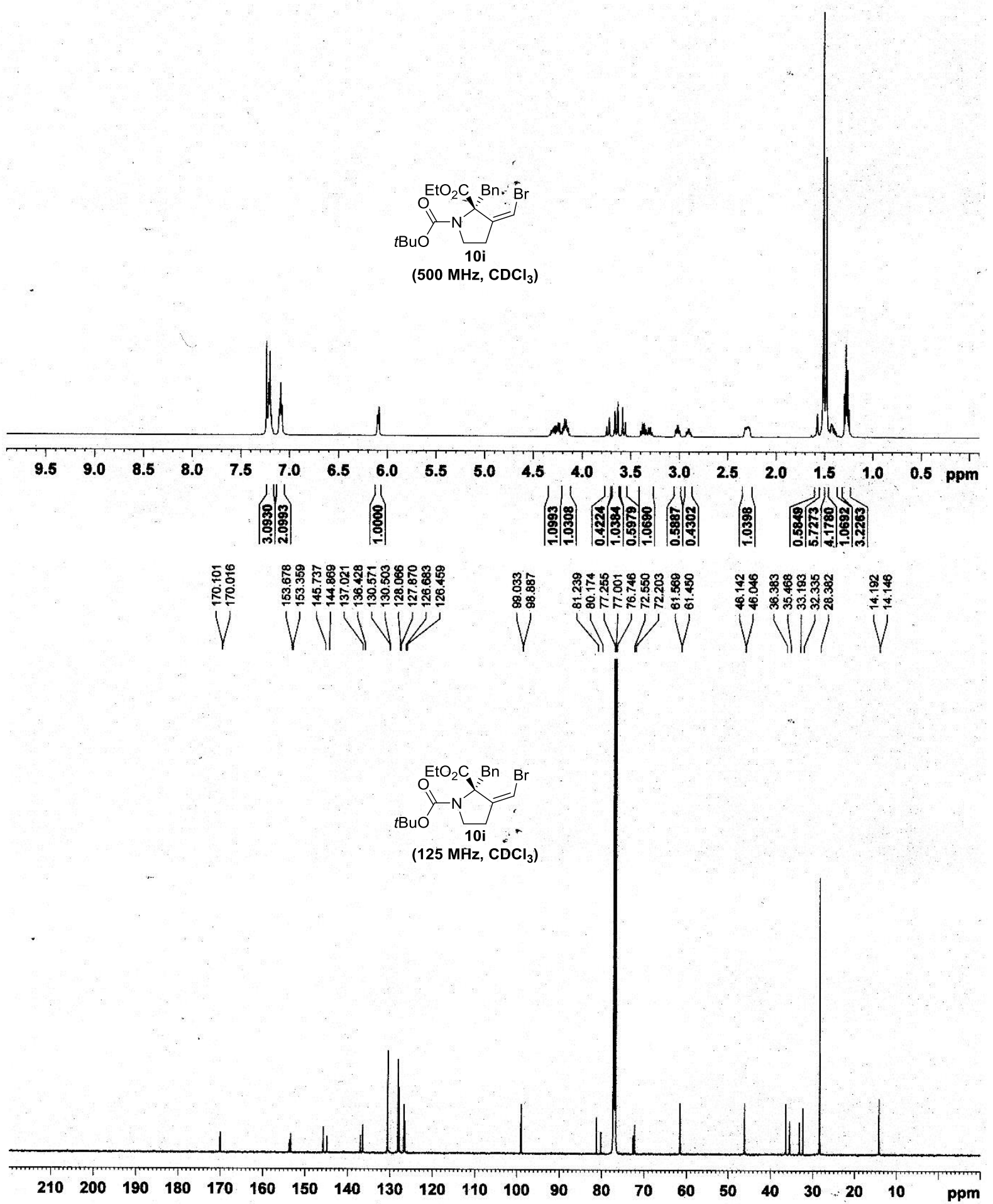


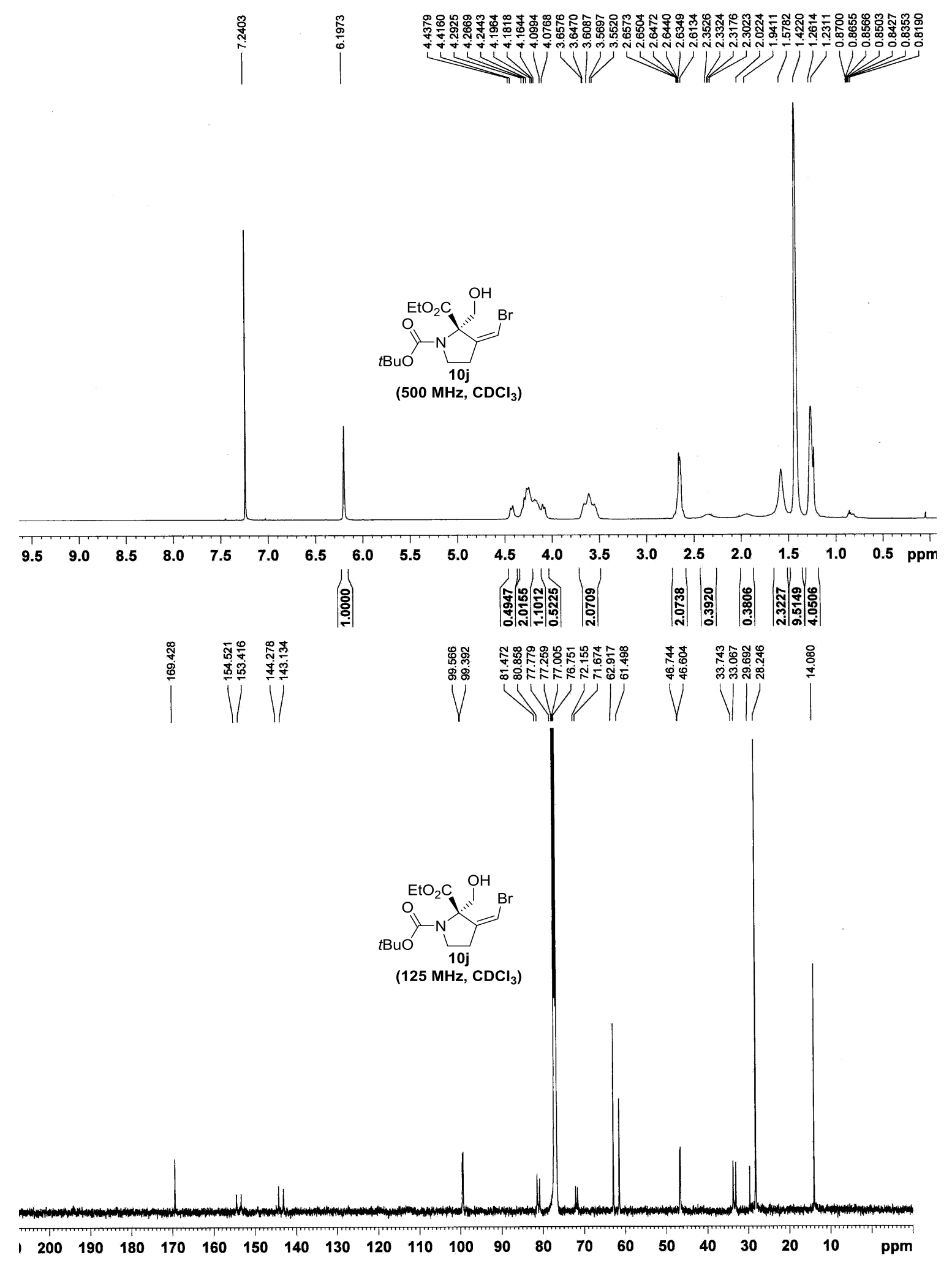



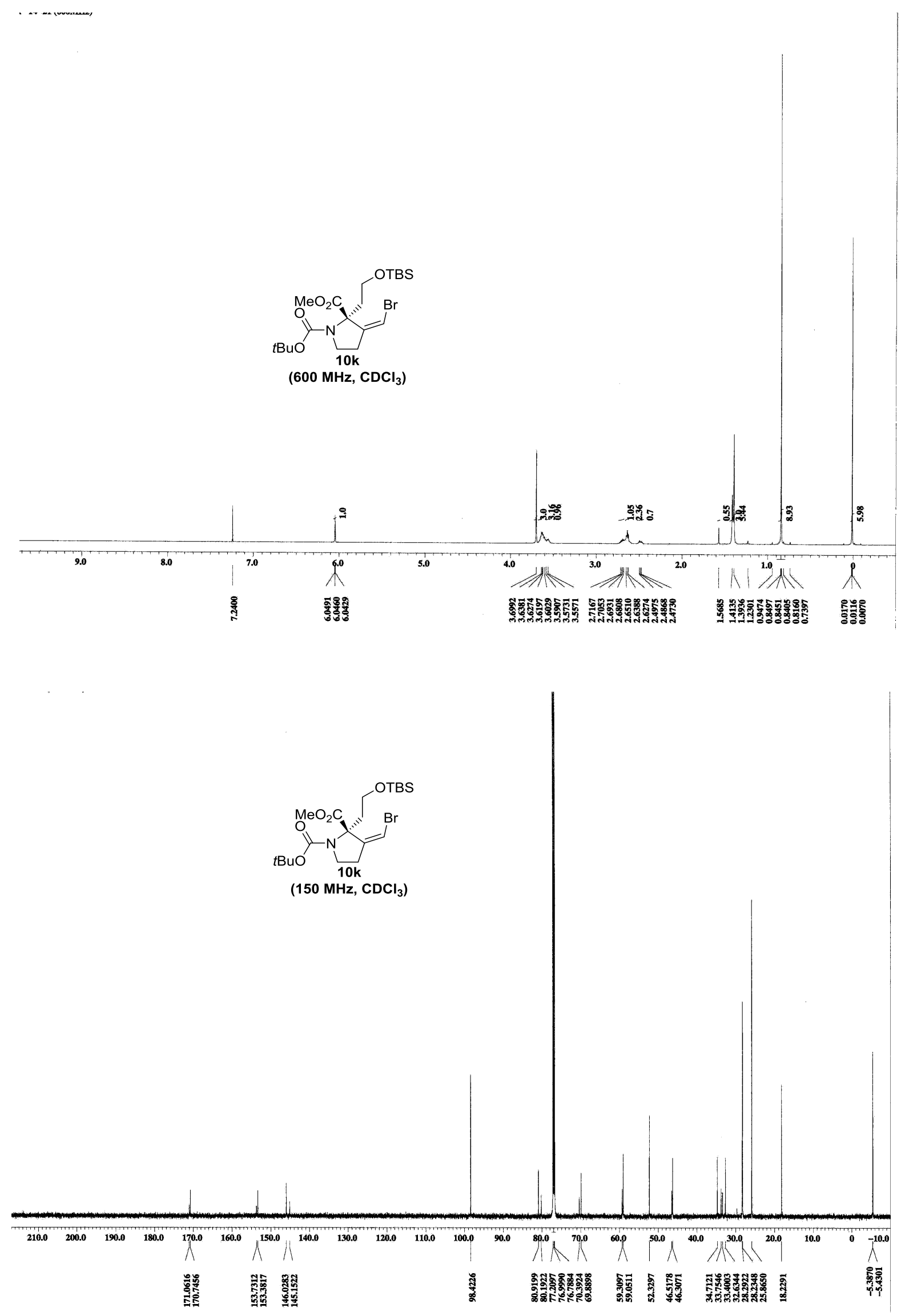

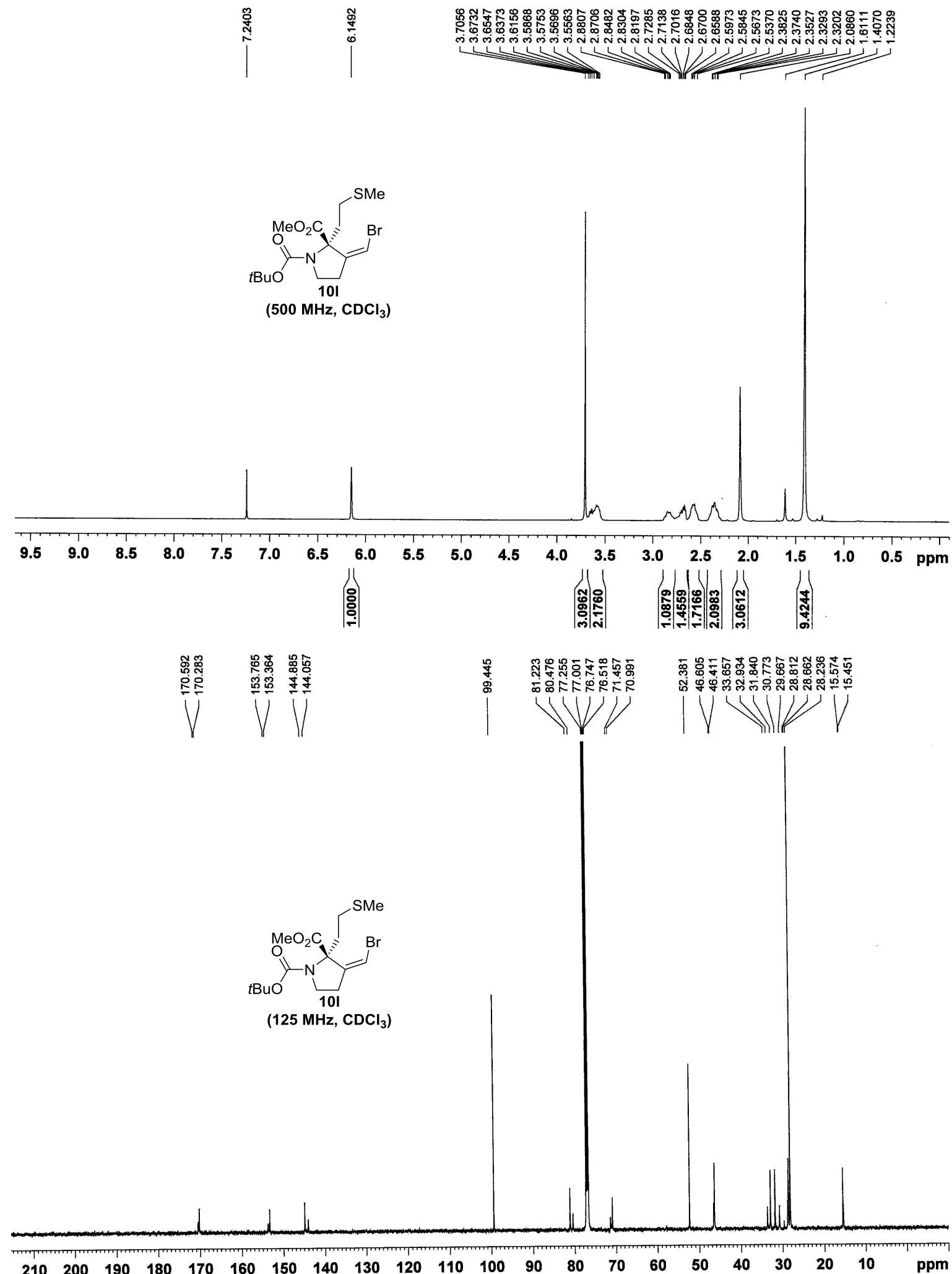


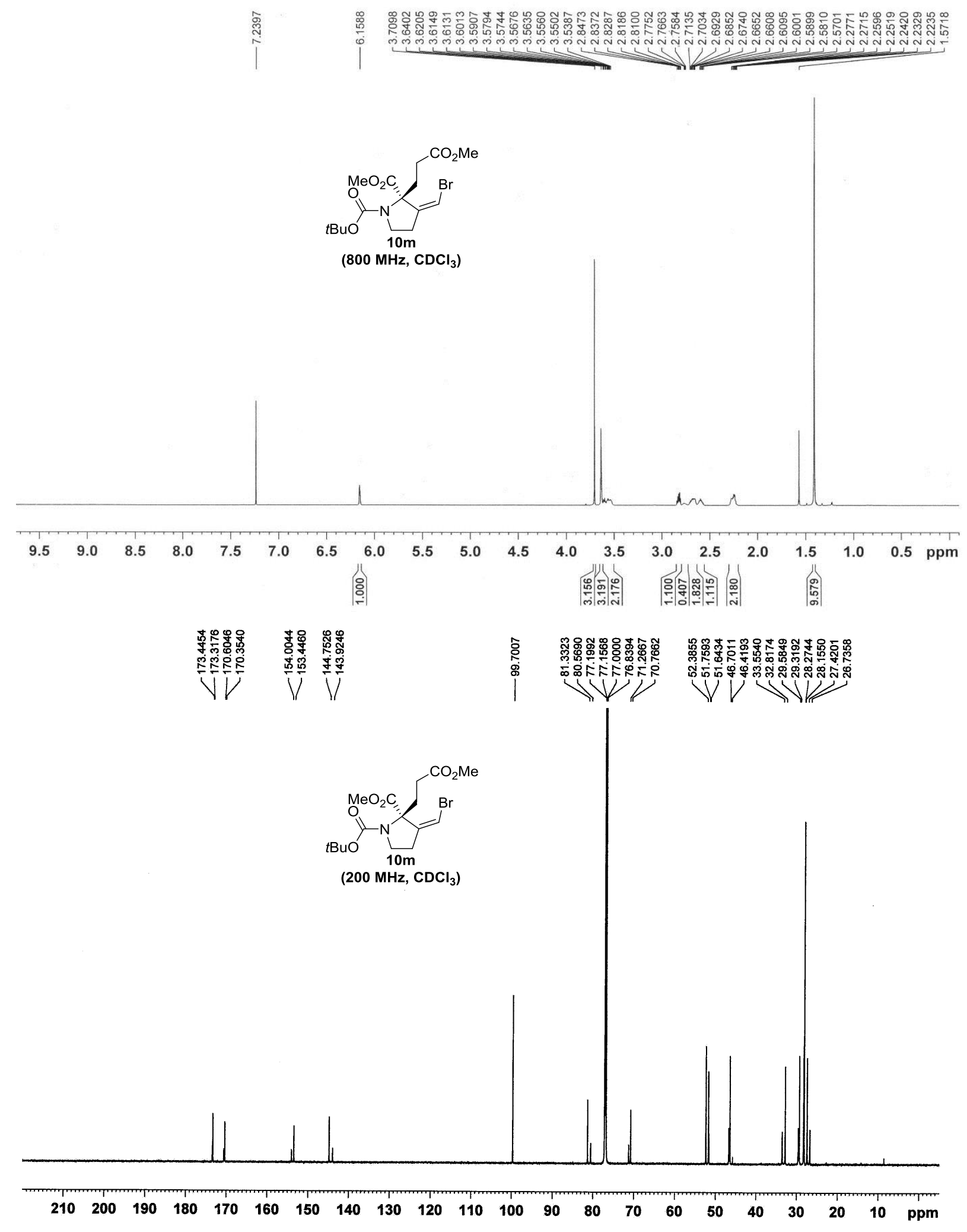



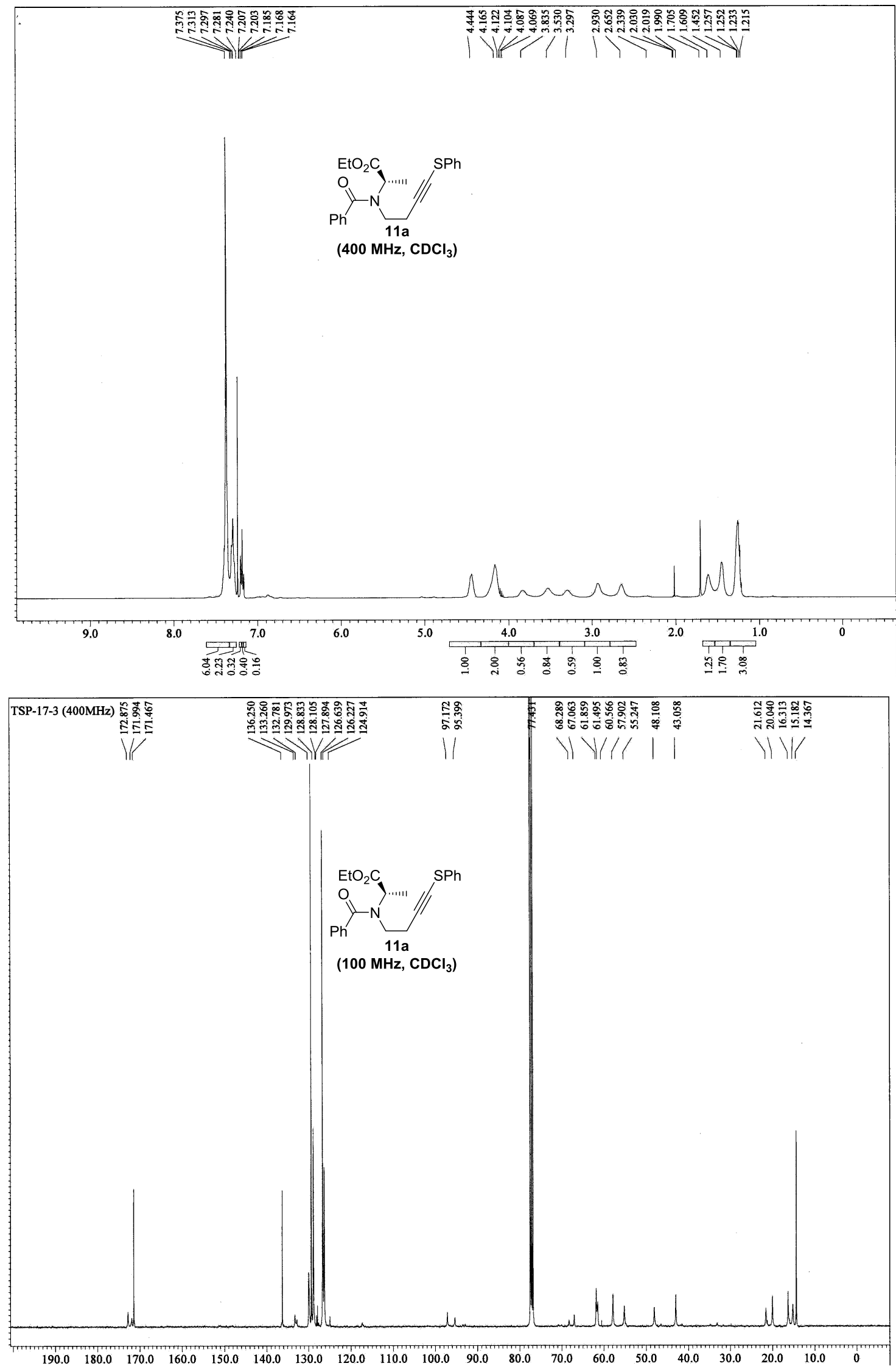


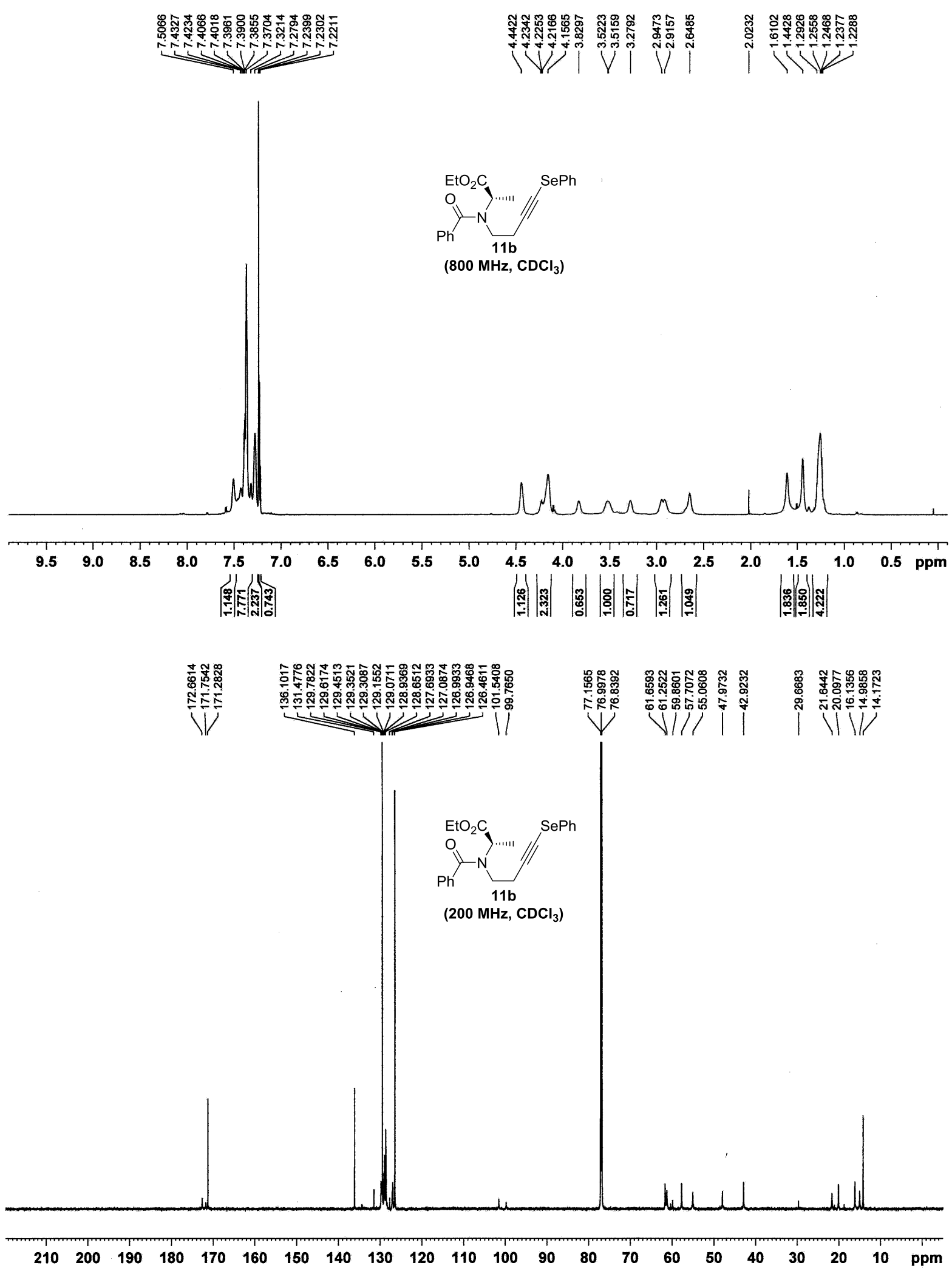



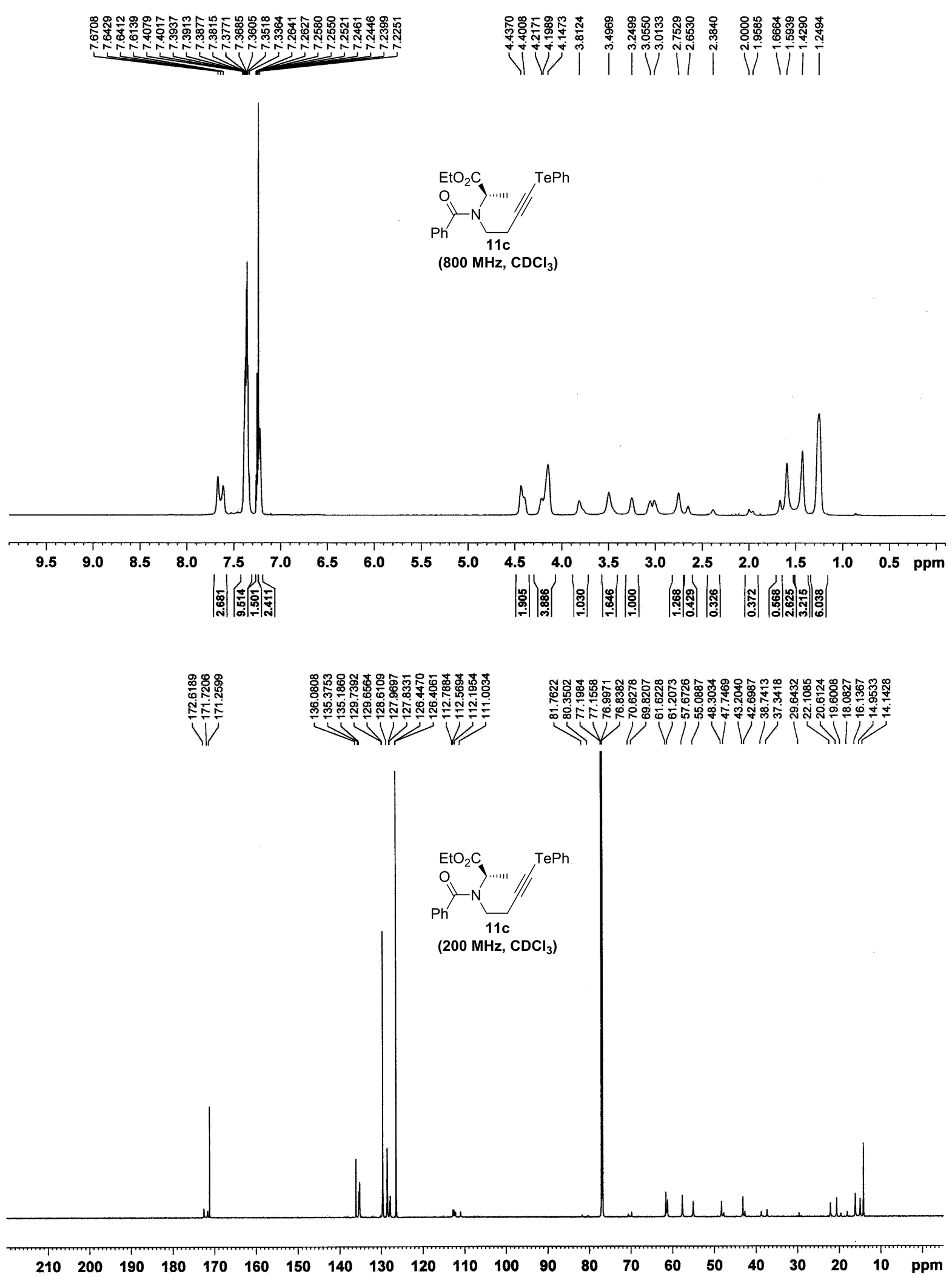

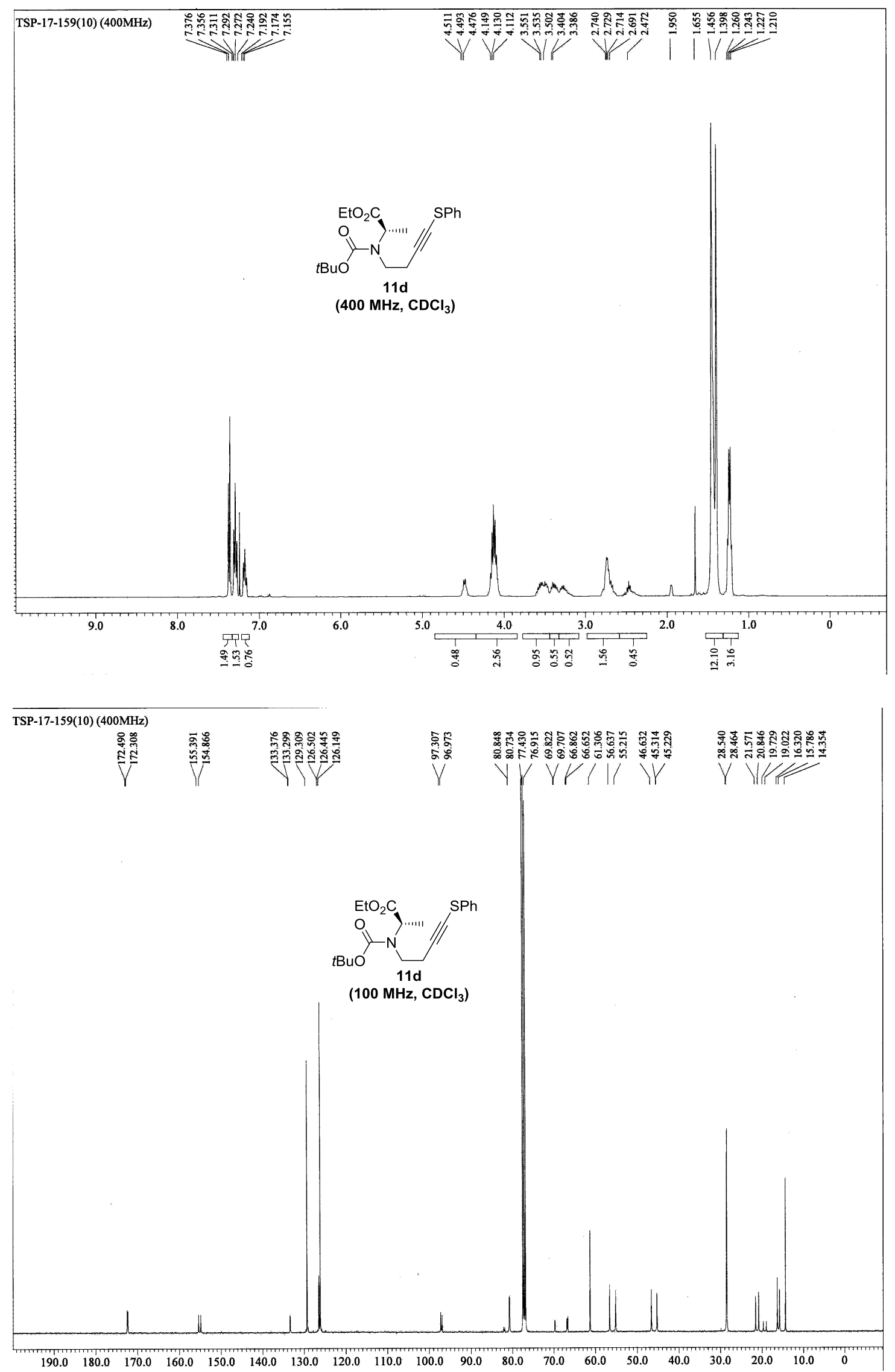

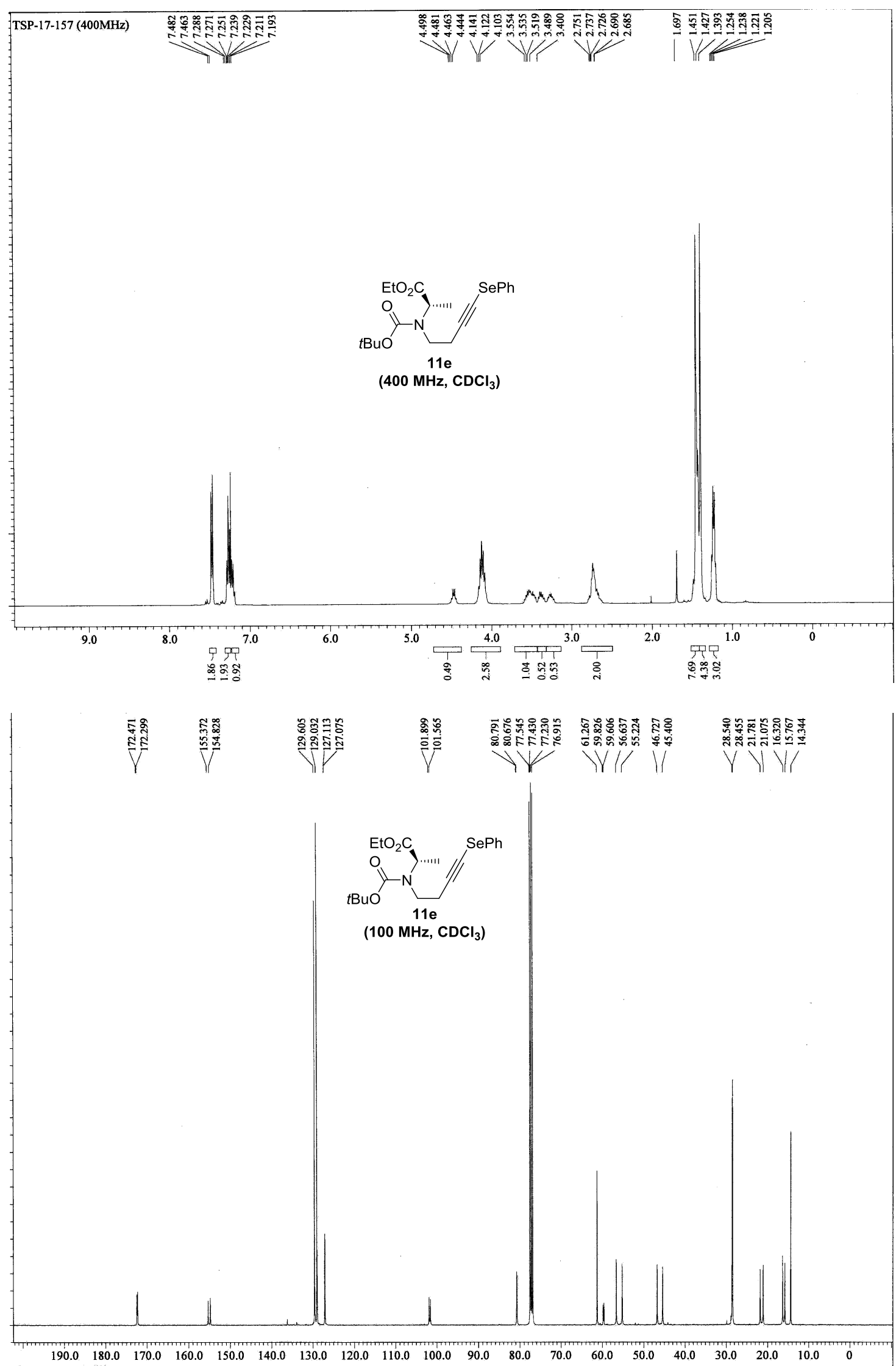


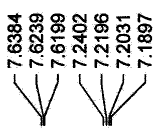

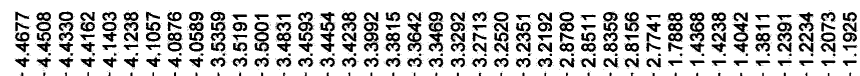

$\rightarrow$

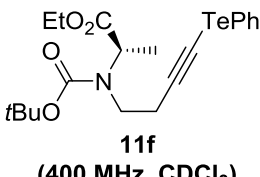

$\left(400 \mathrm{MHz} \mathrm{CDCl}_{3}\right)$
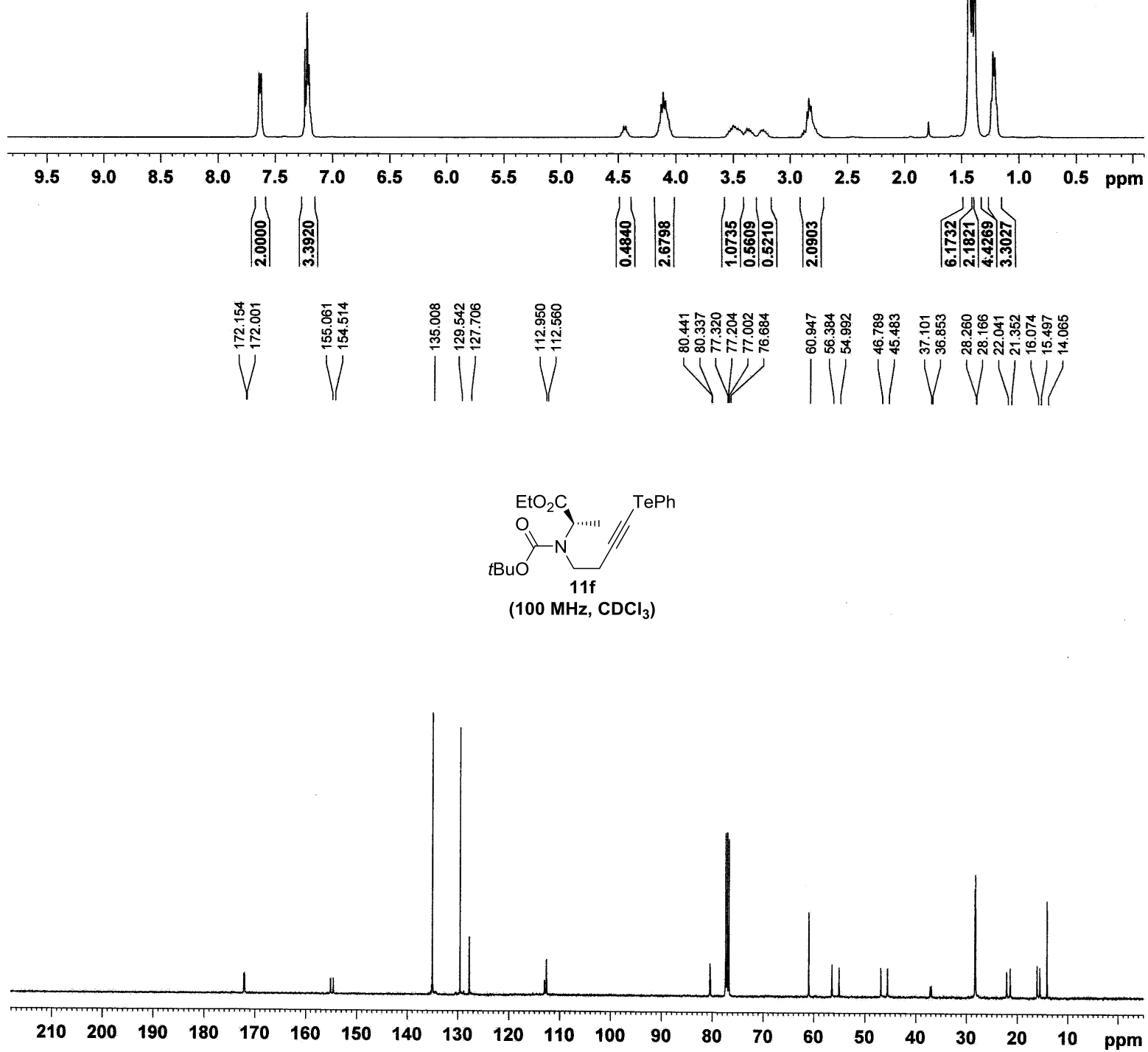


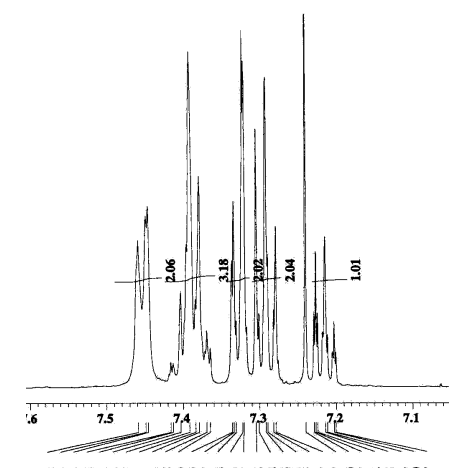

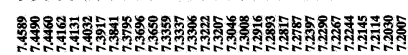

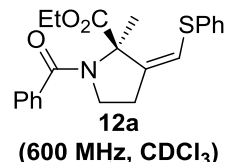

$\left(600 \mathrm{MHz}, \mathrm{CDCl}_{3}\right)$

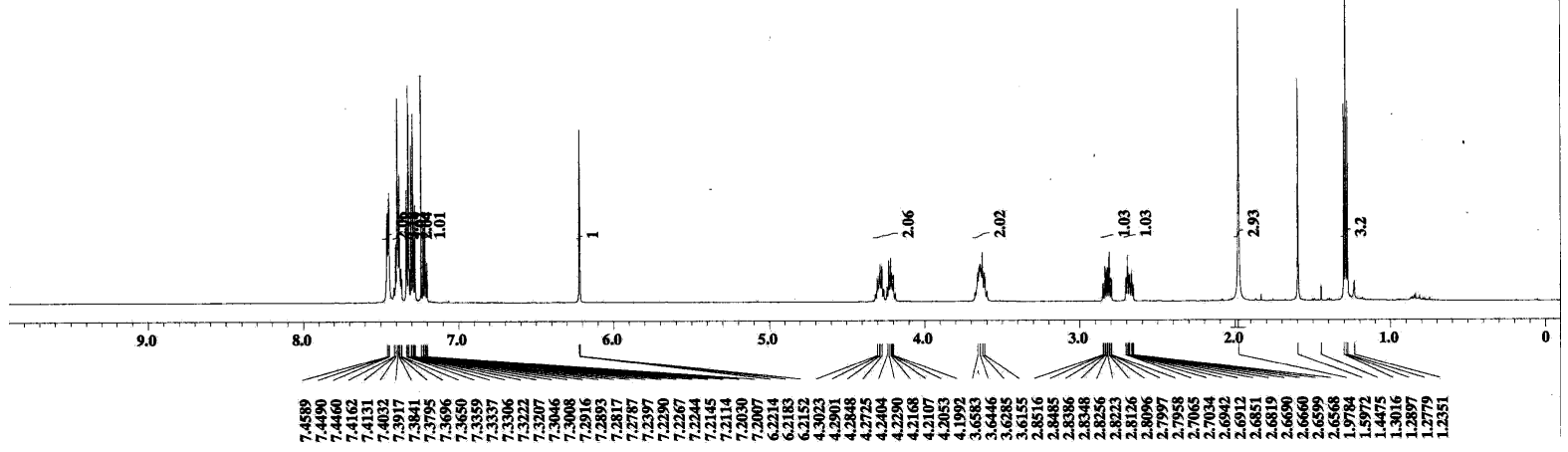

$\overline{\text { rSP-16-191 (600MHz) }}$
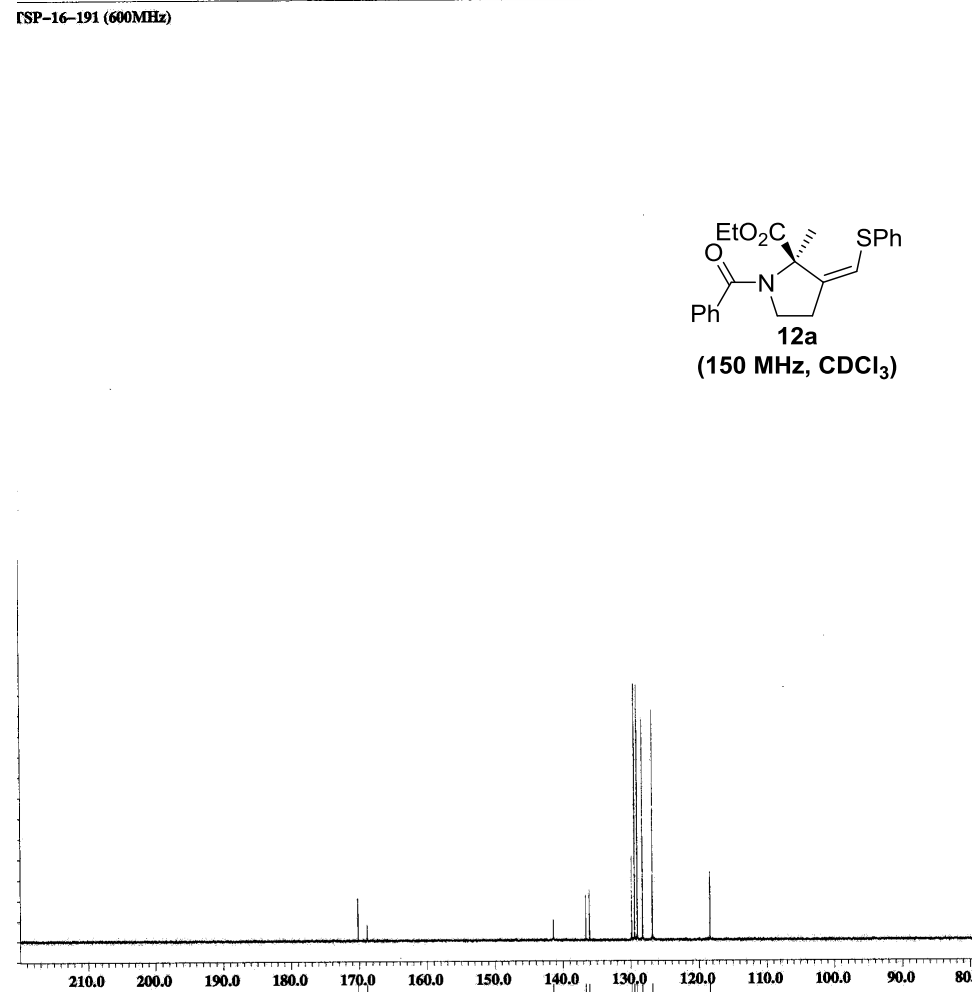

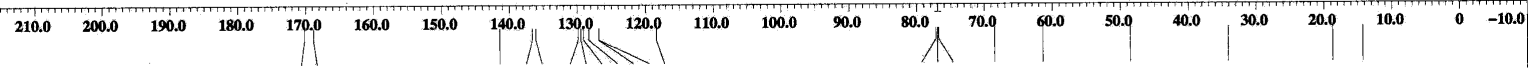

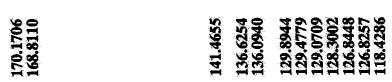

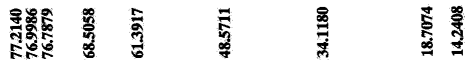



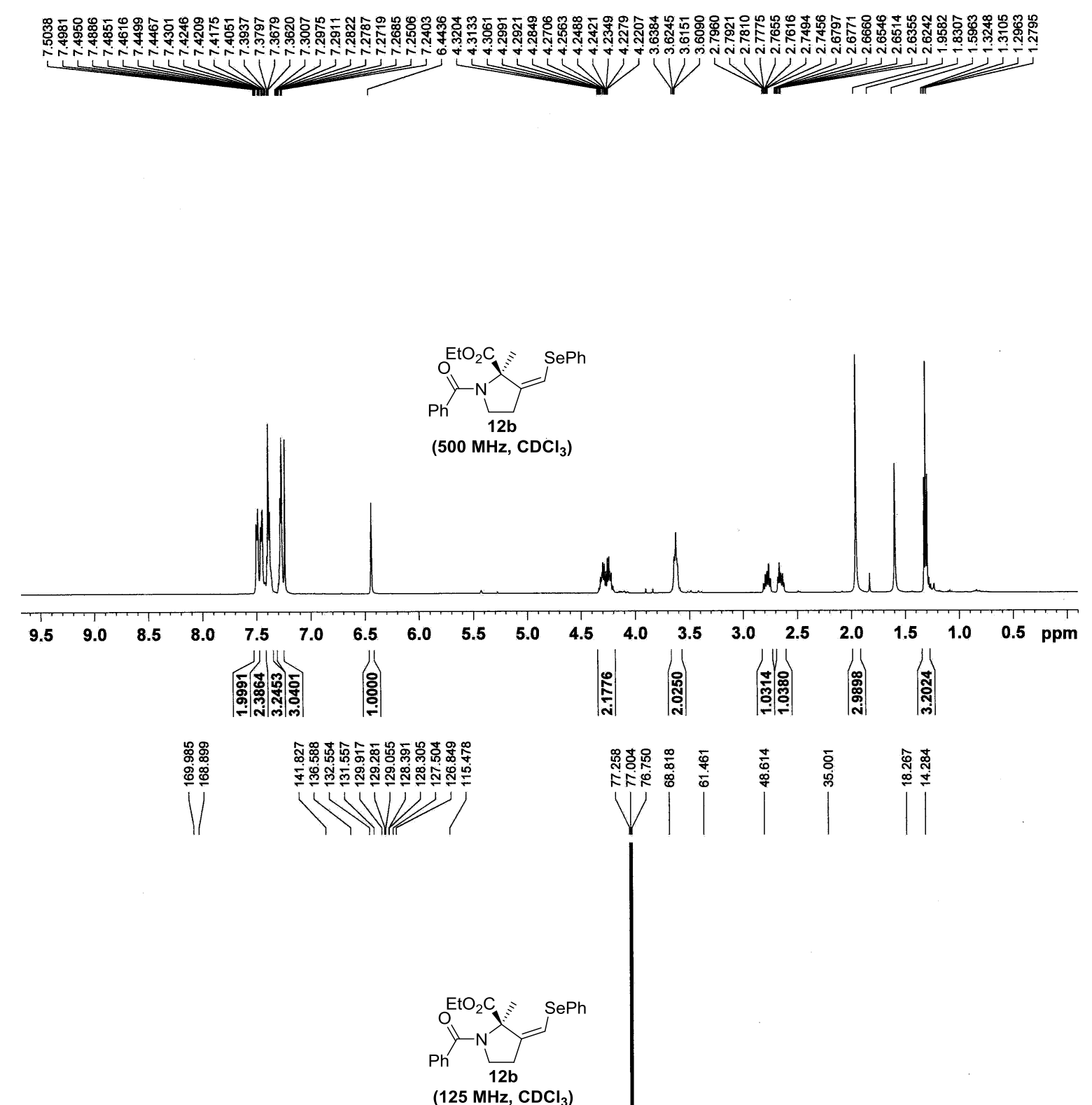

(125 MHz, $\mathrm{CDCl}_{3}$ )

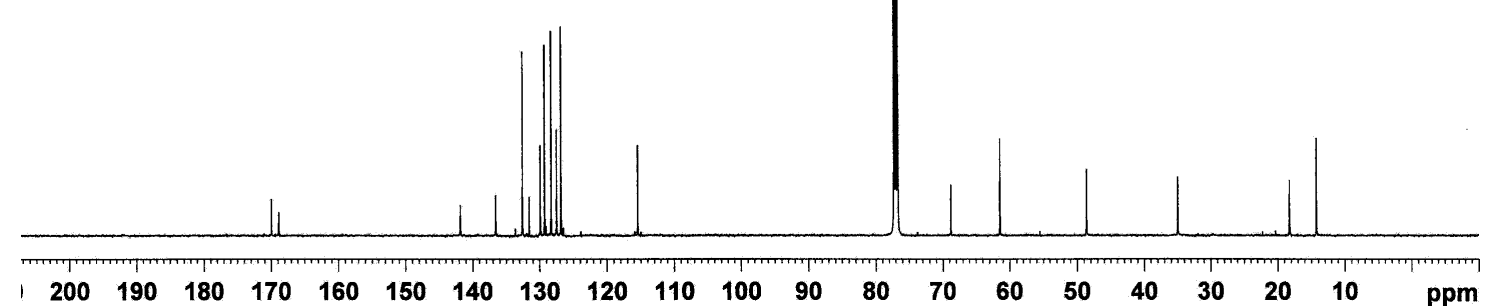



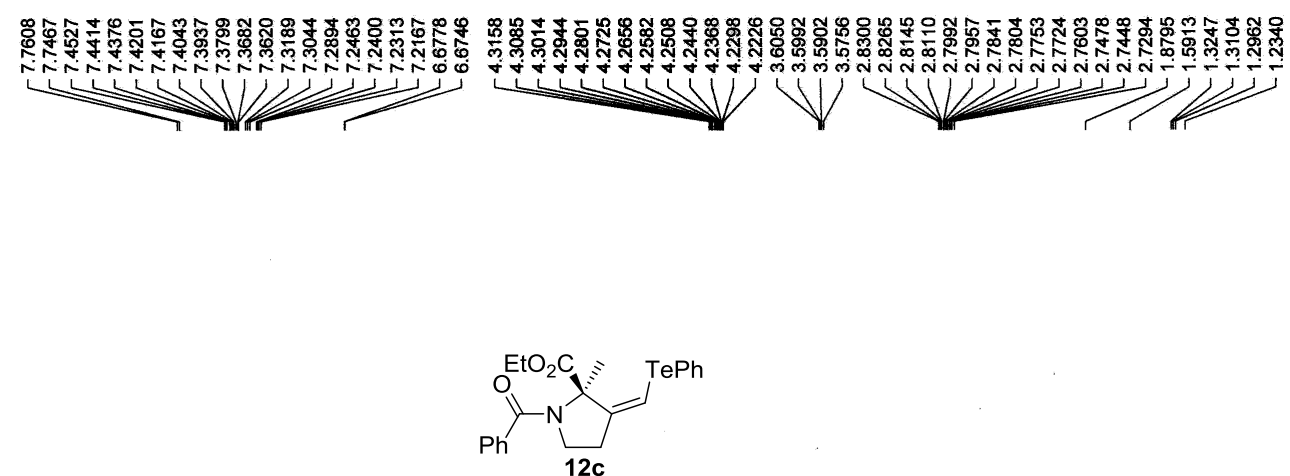

(500 MHz, $\mathrm{CDCl}_{3}$ )
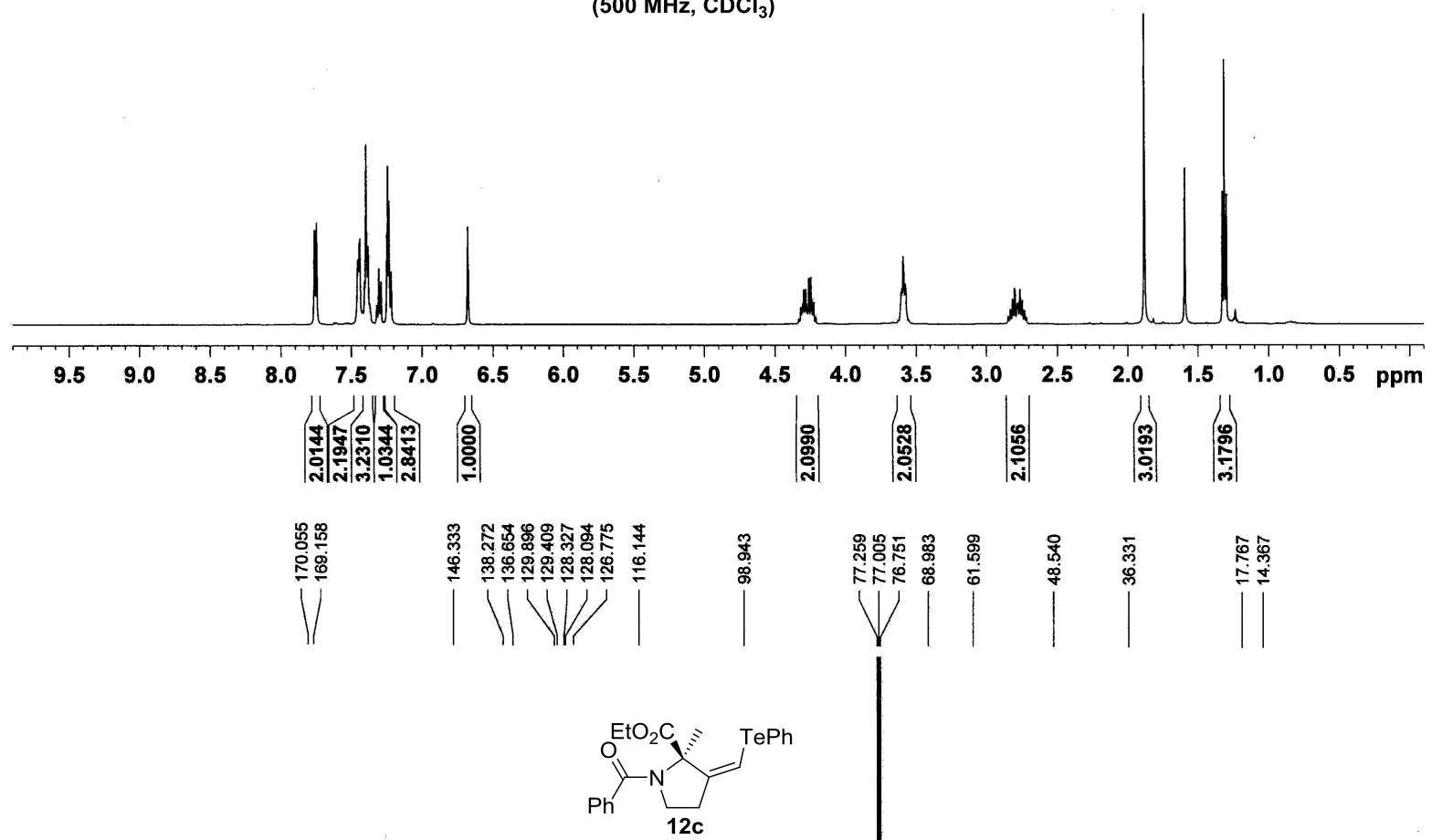

(125 MHz, CDCl ${ }_{3}$ )

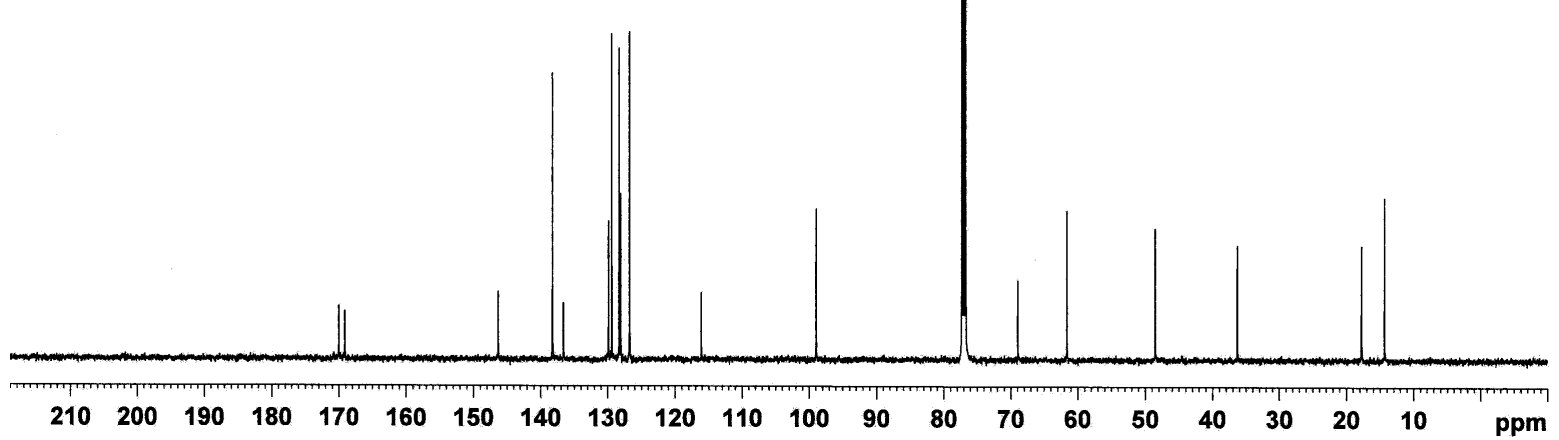




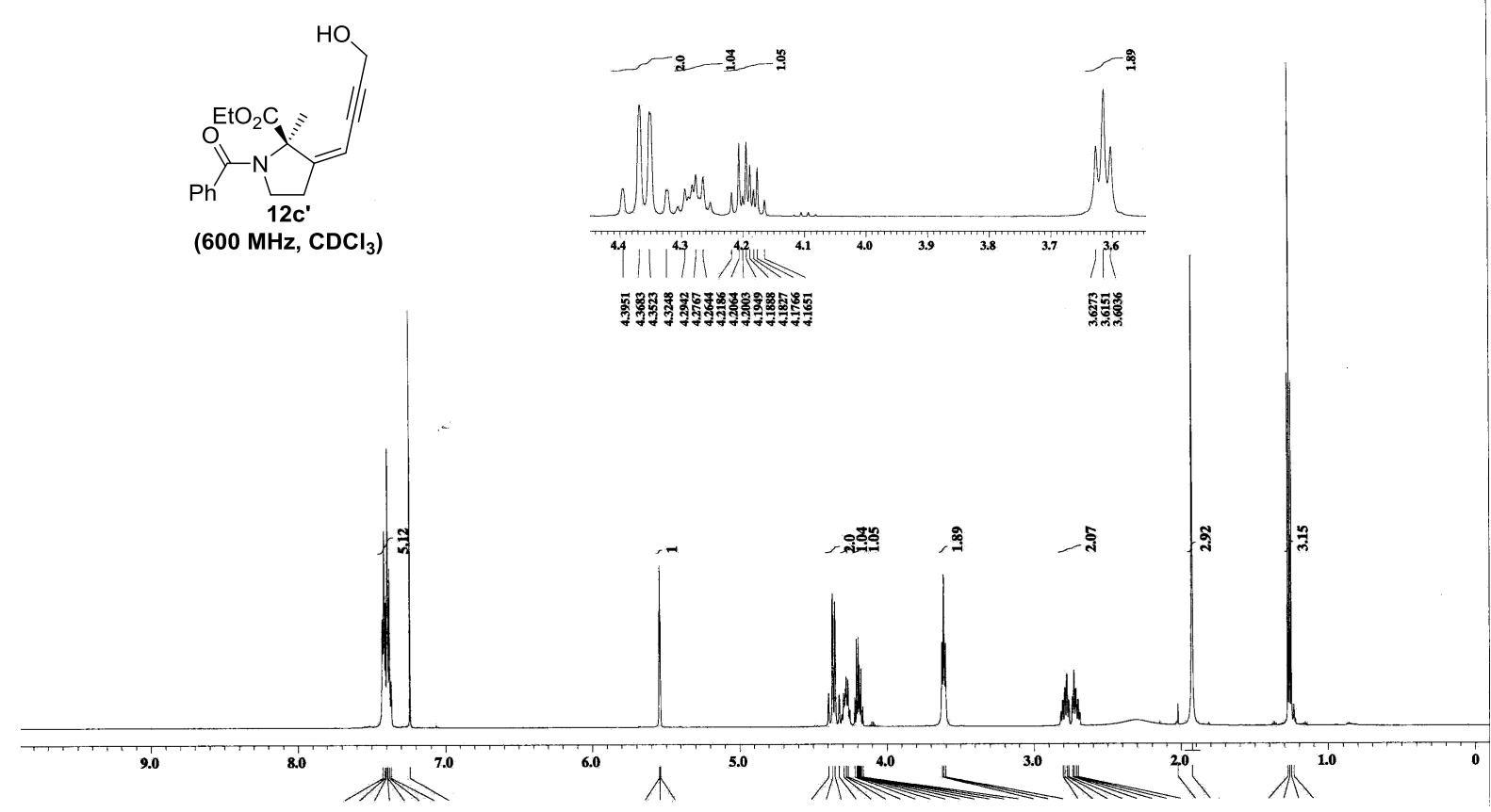

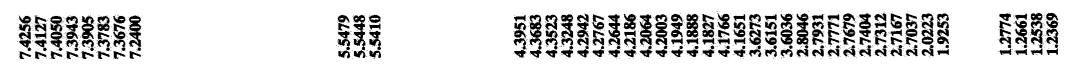
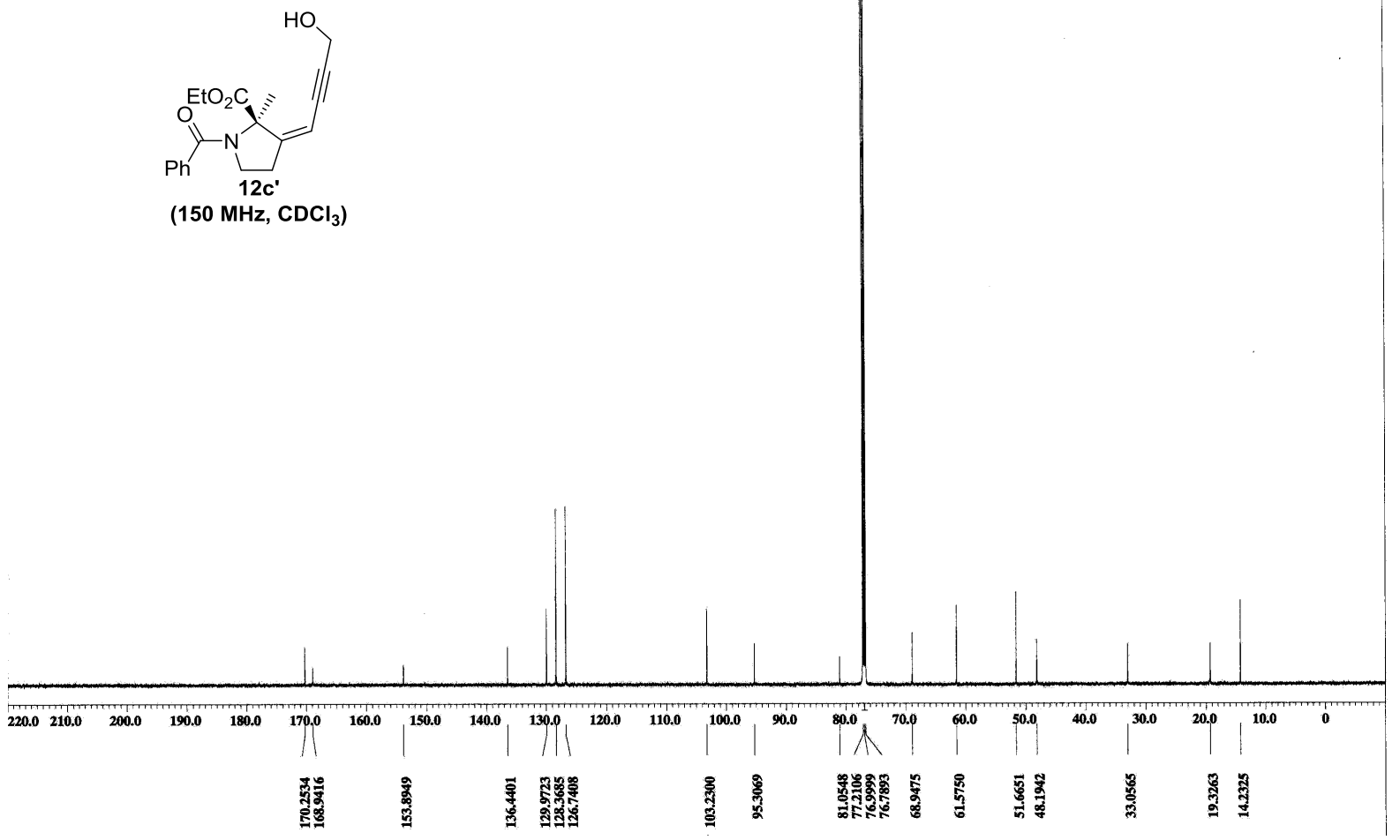

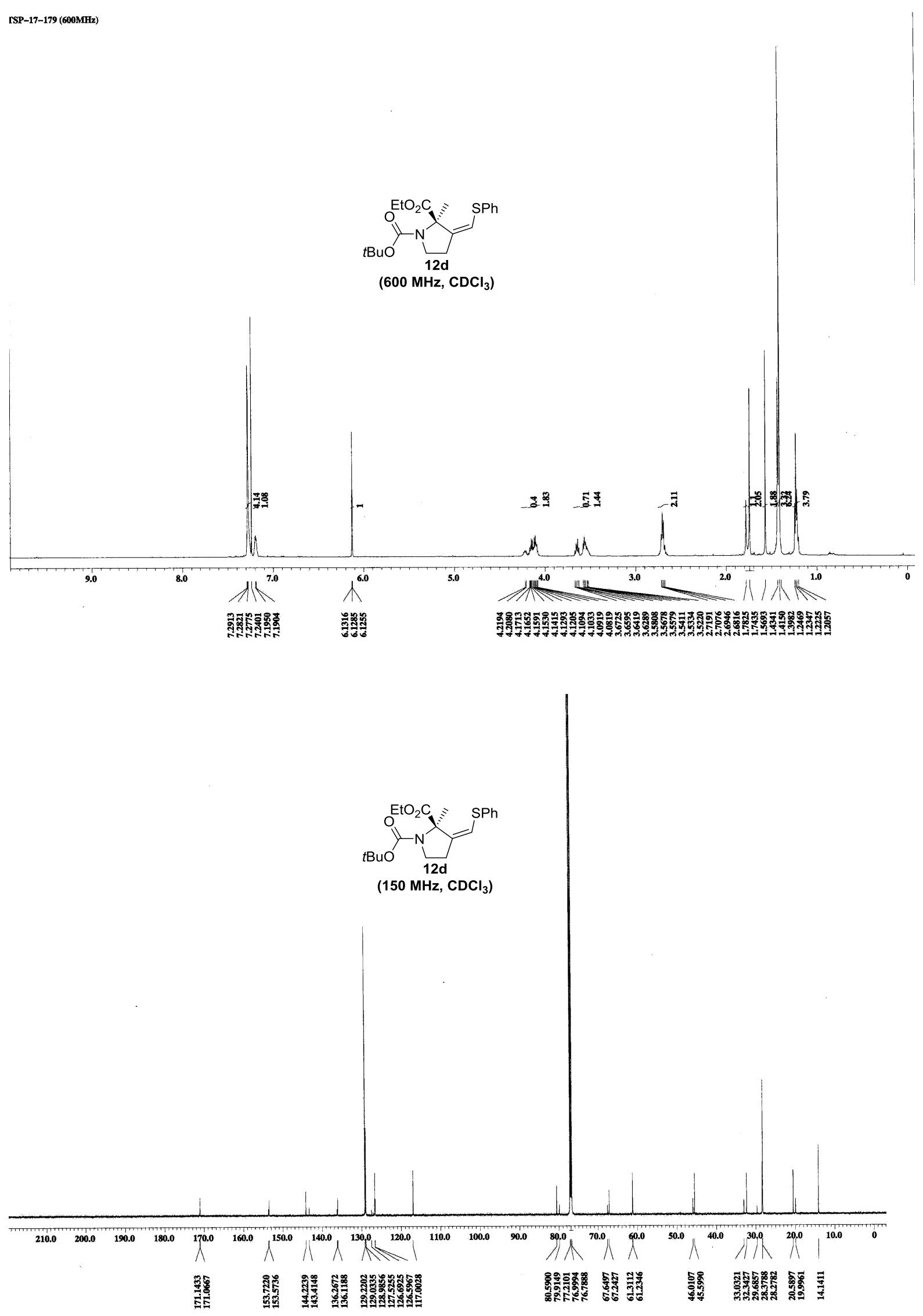

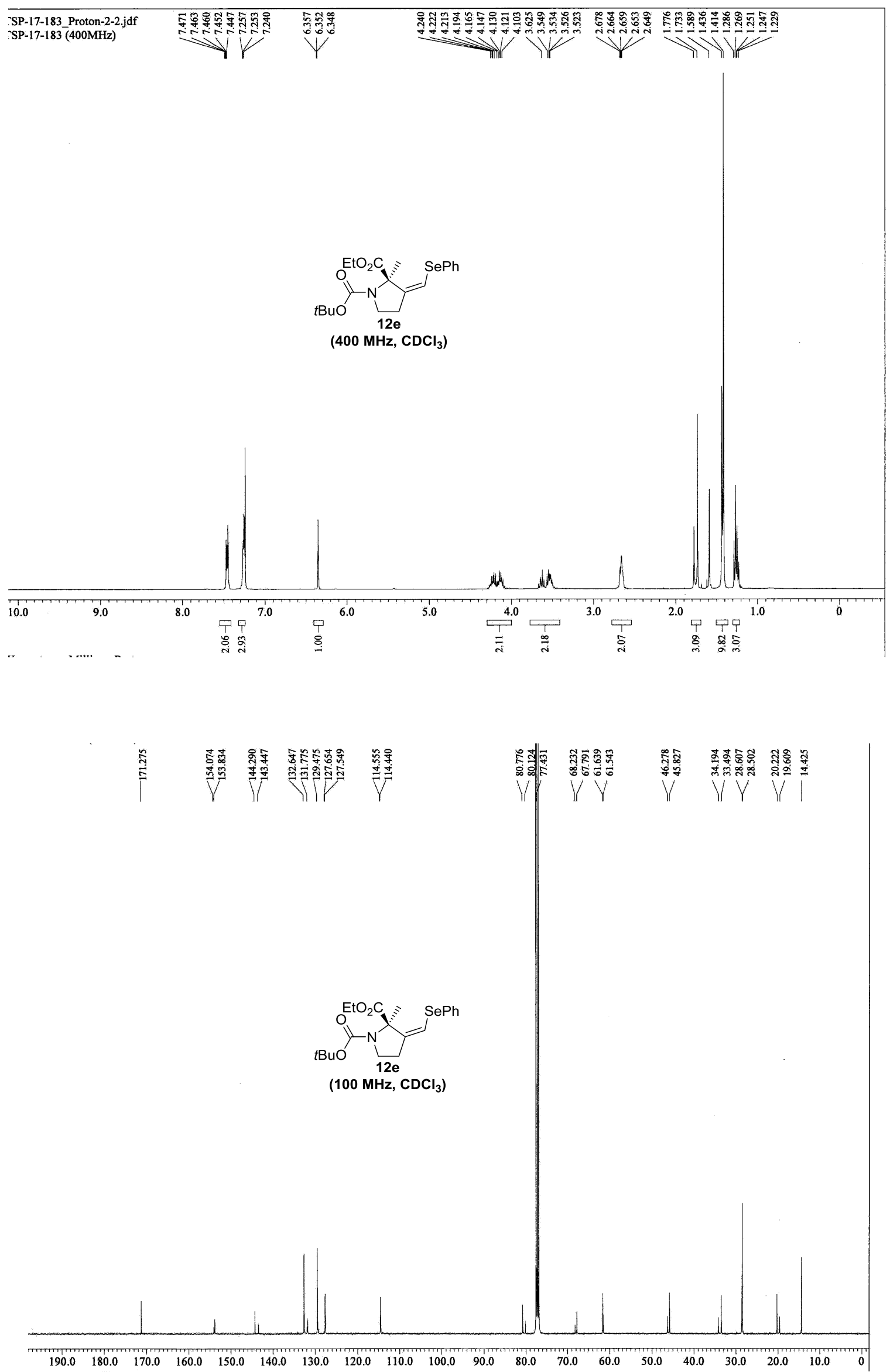

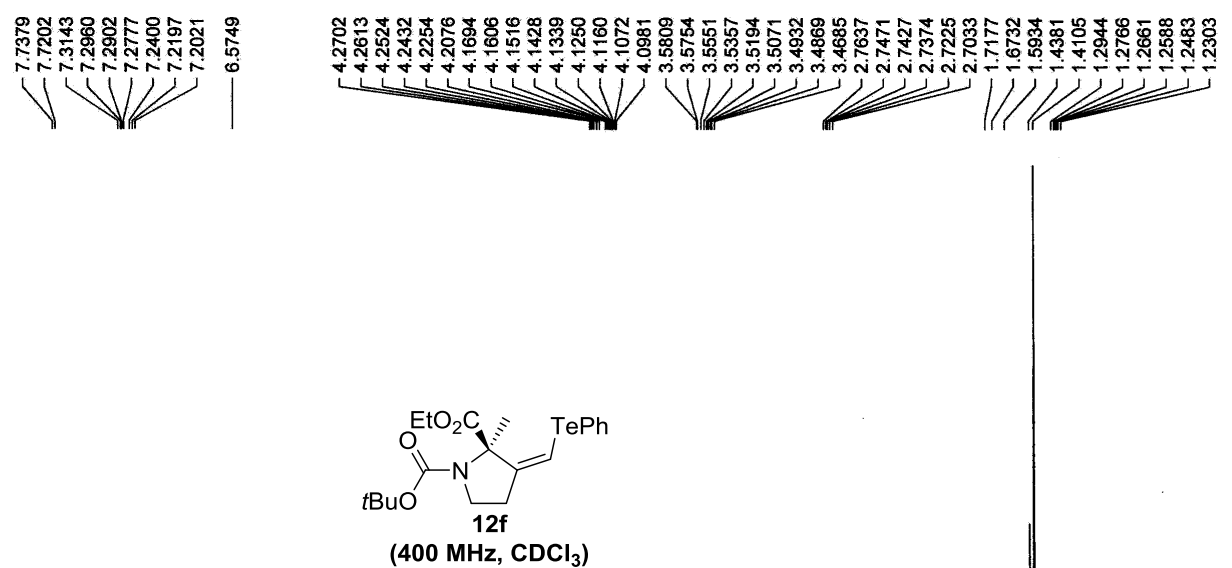

(400 MHz, $\mathrm{CDCl}_{3}$ )
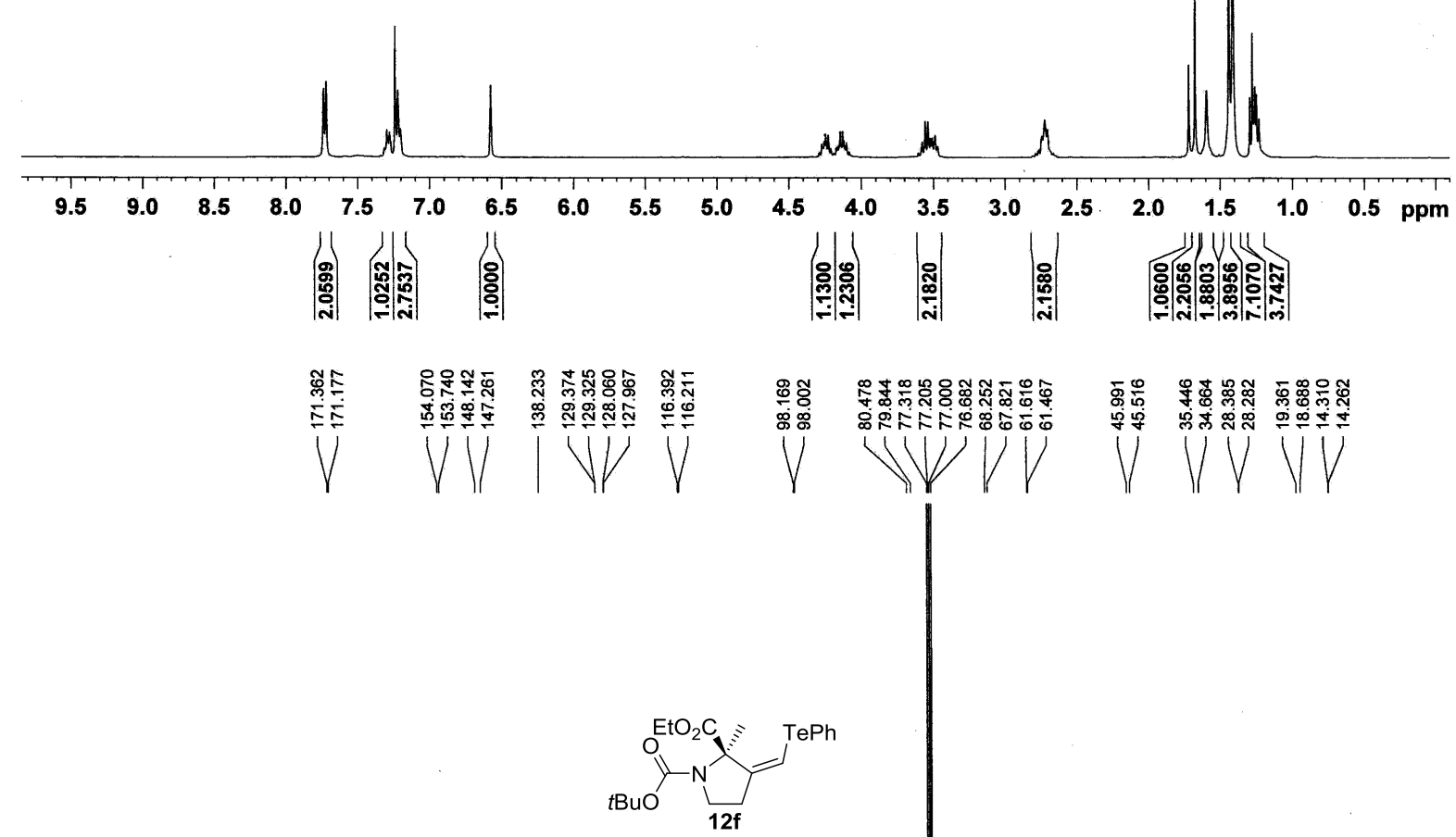

(100 $\mathrm{MHz} \mathrm{CDCl}_{3}$ )

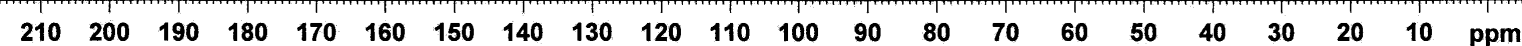

